\title{
Abstracts of the WASAD Congress 2021: an International Congress of the World Association for Stress Related and Anxiety Disorders, held on 20-22 September 2021 in Vienna, Austria
}

1

Specific appraisals, unspecific physiology: HPA and SAM axis as correlates of subjective stress, threat, and challenge in daily life

Dr. Maurizio Sicorello ${ }^{1}$, Andreas B. Neubauer ${ }^{2}$, Martin Stoffel ${ }^{3}$, Friederike Köhler ${ }^{3}$, Andreas Voss ${ }^{3}$, Beate Ditzen ${ }^{3}$

${ }^{1}$ Central Institute of Mental Health, Mannheim, Germany, ${ }^{2}$ DIPF | Leibniz Institute for Research and Information in Education, Department for Education and Human Development, Frankfurt, Germany, ${ }^{3}$ Heidelberg University, Heidelberg, Germany

Stress was originally conceptualized as an unspecific response to anything interfering with an organism's homeostasis, HPA and SAM axis activity being the central physiological systems. In contrast, the dominant psychological stress theory by Lazarus and Folkman proposed two different stress appraisals - threat and challenge-which likely differ in their implications for motivation, emotion, performance, and health. In the attempt to relate threat and challenge appraisals to stress physiology, many possible assertions have been made: the cortisol stress response might be equally involved in both appraisals, asymmetrically more active during threat, or exclusively active during threat. We tested whether the psychoneuroendocrinology of stress appraisals can also be investigated in naturalistic transcontextual everyday life settings. Forty-two healthy participants submitted 25 saliva samples over the course of a week with concurrent short questionnaires on momentary stress appraisals and mood. Momentary cortisol and alpha amylase were assessed as markers of HPA and SAM axis activity. Moreover, we used within-person response surface analyses to elucidate the role of demands-resources mismatch, the main determinant of stress appraisal. We found that both momentary cortisol and alpha amylase were robustly associated with subjective stress and appeared to be unspecific for threat and challenge. We present these results and demonstrate how the methodology of within-person response surface analysis can aid research on stress appraisal.

\section{2}

The moderating role of resilience on the relationship between the psychological impact of the COVID-19 crisis and mental health: A structural equation model approach

Mag. Martha Schneider ${ }^{1}$, Dr. Claudia Traunmüller ${ }^{2}$, Mag. Rene Stefitz $^{3}$, Prof. Dr. Andreas Schwerdtfeger ${ }^{4}$

${ }^{1}$ Central Institute of Mental Health, Mannheim, Germany, ${ }^{2}$ DIPF | Leibniz Institute for Research and Information in Education, Department for Education and Human Development, Frankfurt, Germany, ${ }^{3}$ Heidelberg University, Heidelberg, Germany

Background: Psychological resilience is considered to constitute an important factor for protecting mental health, especially during times of crises, like the COVID-19 pandemic outbreak. However, there is a lack of research on the potential buffering effect of resilience on the psychological impact of COVID-19 as related to mental health. The aim of the current study was to examine the protective effects of resilience on mental health during the initial stage of the COVID-19 outbreak in Austria.

Methods: Analysis was based on data collected from 4113 Austrian residents (74\% female, age: $\mathrm{M}=38.68, \mathrm{SD}=13.36$ ), who participated in an anonymous online survey. The survey addressed sociodemographic data, the subjective response to COVID-19 (Impact of Event Scale; IES-R), mental health status (Depression Anxiety Stress Scale; DASS-21), and resilience (Resilience-Scale; RS-11).

Results: Structural equation modelling showed significant positive associations between the IES-R score and depressive symptoms, stress, and anxiety, respectively. Resilience was significantly negatively associated with depression, stress, and anxiety. Furthermore, resilience moderated the relation between the impact of COVID-19 and anxiety symptoms. However, there was no moderating effect of resilience on the relationship between IES-R and both depression and stress.

Conclusion: The psychological impact of COVID-19 on anxiety symptoms seem to vary with the level of resilience. Longitudinal studies are needed to investigate the temporal relationship between resilience, mental health, and the psychological impact of the COVID-19 crisis. 
3

\section{Can using music for relaxing purposes impact stress recovery?}

\author{
Yichen Song ${ }^{1}, \mathrm{PhD}$ Nadine Skoluda ${ }^{1}, \mathrm{PhD}$ Urs Nater ${ }^{1}$ \\ ${ }^{1}$ Clinical Psychology of Adulthood, Faculty of Psychology, \\ University Of Vienna, Vienna, Austria
}

Background: Studies have suggested that music can reduce psychological and biological responses to a stressor, but it is unclear whether music has the same effect on stress recovery. Field studies indicate that it is very common for individuals to use music in daily life for the specific purpose of relaxation. In a lab-based study, we wanted to explore whether people who are generally using music for relaxation purposes would indeed show improved recovery from an acute stressor.

Methods: Sixty-two healthy male participants (Mage $=24.03$, SDage $=3.20$ ) were separated into two groups, based on their general use of music. Group $1(\mathrm{~N}=20)$ indicated frequent use of music for relaxation purposes, whereas Group $2(\mathrm{~N}=42)$ did not. All subjects underwent the Trier Social Stress Test for Groups, and saliva samples were collected 9 times during the whole process of stress induction and recovery. The levels of cortisol and saliva alpha-amylase (sAA) were analyzed from saliva samples.

Results: Findings indicated that during recovery, sAA levels in Group 1 were significantly higher than those in Group $2(\mathrm{t}(57.76)=2.37$, $\mathrm{p}=.021, \mathrm{~d}=0.55)$, whereas there were no differences in cortisol levels. Further exploration showed that more use of music for relaxation purposes was associated with more chronic stress, as measured by the Perceived Stress Scale $(\mathrm{r}(61)=.234, \mathrm{p}=.023)$.

Conclusions: Contrary to our expectations, our results indicate that listening to music for relaxation purposes does not result in improved recovery from a stressor.

\section{4}

\section{Role of Npbwr1 in mood and major depressive disorder}

\section{Dr. Olivia Engmann ${ }^{1}$, Janine Aly ${ }^{1}$, Prof. Dr. Christian Hübner ${ }^{1}$ \\ ${ }^{1}$ Jena University Hospital, Jena, Germany}

Depression is characterized by low mood, fatigue, sleep problems and altered day-and-night rhythms. Our project explores a previously undescribed neural pathway, which integrates the effects of circadian time and caffeine on improved mood. Specifically, we found that the receptor for neuropeptides $\mathrm{B}$ and $\mathrm{W}, \mathrm{Npbwr} 1$, is rapidly regulated by caffeine when mood improvements occur. Npbwr1 levels are increased in a mouse model of depression. Characterizing the Npbwr1 pathway and its role in depressive-like states and mood further will provide us with a better understanding of molecular mechanisms underlying mood disorders such as depression.

\section{5}

\section{A systematic review of thermosensation and thermoregulation in anxiety disorders}

\author{
Dr. Susanne Fischer ${ }^{1}$, MSc Florence Haas ${ }^{1}$, Dr. Jana Strahler ${ }^{2}$ \\ ${ }^{1}$ University Of Zurich, Clinical Psychology and Psychotherapy, \\ Zurich, Switzerland, ${ }^{2}$ University of Giessen, Psychotherapy \\ and Systems Neuroscience, Giessen, Germany
}

Background: Excessive sweating, chills, hot flushes, and blushing are symptoms frequently reported by patients with anxiety disorders. Given their potential visibility to others, they represent important reinforcers of anxiogenic cognitions and behaviours. One system that may be involved in the manifestation of these symptoms is the thermosensory/thermoregulatory system. The aim of the present study was to find out to what extent patients with anxiety disorders are characterised by abnormalities in this system.

Methods: PubMed and PsycINFO were systematically searched. Studies were eligible if they (i) included an adult sample of patients with an anxiety disorder, (ii) assessed any thermosensation or thermoregulatory effectors/outcomes, and (iii) used a case-control study design.

Results: A total of 86 studies were identified. There was no evidence of abnormal thermosensation in patients with anxiety disorders. Patients with specific phobias showed more pronounced sweating upon confrontation with phobic stimuli. Patients with social anxiety disorders exhibited altered vasodilatory responses and increased sweating during in sensu exposure to phobic situations. Patients with panic disorder were characterised by increased daily sweating as well as increased sweating in response to various stimuli. There was evidence of altered skin temperature in all three conditions.

Conclusions: In patients with social anxiety disorder, excessive sweating, hot flushes, and blushing may be paralleled by specific vasomotor and sudomotor alterations. In panic disorder, a subgroup of patients may be characterised by a more general propensity to sweat. (Micro-)longitudinal research is warranted to investigate whether this represents a vulnerability to the experience of panic attacks.

6

\section{Gelotophobia and the brain: neural correlates during social signal processing}

Benjamin Kreifelts ${ }^{1}$, Ariane Wiegand ${ }^{1}$, Thomas Ethofer ${ }^{1,2}$, Carolin Brück $^{1}$, Heike Jacob ${ }^{1}$, Michael Erb ${ }^{2}$, Dirk Wildgruber ${ }^{1}$

${ }^{1}$ Department of Psychiatry and Psychotherapy, Eberhard-KarlsUniversity of Tübingen, Tübingen, Germany, ${ }^{2}$ Department of Biomagnetic Resonance, Eberhard-Karls-University of Tübingen, Tübingen, Germany

Laughter is a very frequent social signal with a predominantly positive emotional connotation. However, beyond social inclusion, it can also communicate social exclusion. Interindividually, the perception of laughter is highly variable and can be phobic in extreme cases. This phobia is termed gelotophobia which is defined as the specific fear of being laughed at. First clinical studies indicate an increased co-occurrence of this phobia with various psychiatric disorders and corroborate a close relationship with social anxiety. However, the neural representation of this fear remains completely unexplored to date.

Here, we report a first functional magnetic resonance imaging (fMRI) study aiming to delineate the neural representation of gelotophobia during voice and face processing in 67 individuals.

The results revealed positive correlations between the individual severity of gelotophobic symptoms and voice-selective responses in the right temporal voice area (TVA), a central node of the cerebral voice processing system, and in the left amygdala. In contrast, faceselective responses were not associated with gelotophobia. Furthermore, the correlation between the severity of gelotophobic symptoms and voice-selective responses in the right TVA remained significant even after accounting for measures of general as well as social anxiety or depressive symptoms. This was not the case in the amygdala where 
the association of voice-selective responses with gelotophobia was not significantly dissociable from that with social anxiety.

Taken together, our study not only offers a first account of the neural representation of gelotophia but also highlights the central role of voices and voice processing for this condition and its neural underpinnings. In particular, the response patterns of the right TVA point to a specific contribution of this central voice processing node not attributable to concomitant symptoms of social or general anxiety or depression.

\section{7}

\section{Acute biological stress reactivity and recovery influences intrusive memory development in healthy men}

MSc Charlotte Elize Hilberdink ${ }^{1}$, Dr. Susanne R. de Rooij ${ }^{2}$, Prof. Dr. Miranda Olff ${ }^{1,3}$, Dr. Jos A. Bosch ${ }^{4,5}$, Dr. Mirjam van Zuiden ${ }^{1}$

${ }^{1}$ Amsterdam UMC-AMC; Department of Psychiatry, Amsterdam, The Netherlands, ${ }^{2}$ Amsterdam UMC-AMC; Department of Epidemiology and Data Science, Amsterdam, The Netherlands, ${ }^{3}$ ARQ-National Psychotrauma Centre, Diemen, The Netherlands, ${ }^{4}$ University of Amsterdam-Department of Psychology, Amsterdam, The Netherlands, ${ }^{5}$ Amsterdam UMC-University of Amsterdam; Department of Medical Psychology, Amsterdam, The Netherlands

Not all trauma-exposed individuals develop posttraumatic stress disorder (PTSD), denoting differential vulnerability for its development. PTSD's hallmark symptom intrusive memories are thought to result from maladaptive memory consolidation within the first hours posttrauma, leading to poorly contextualized memories prone to automatic retrieval. Severe traumatic stress, can alter memory formation and consolidation processes and decrease context-dependent memory encoding, likely as a consequence of stress-induced activation of the hypothalamic-pituitary-adrenal (HPA) axis and autonomic nervous system. Individual variation in the extent of this acute biological stress reactivity has been found to predict subsequent PTSD symptom development. This could be mediated via its hypothesized impact on contextual memory encoding upon trauma exposure leading to altered likelihood for intrusion development. Understanding the biological and cognitive mechanisms underlying differential vulnerability for PTSD symptom development may facilitate preventive intervention development.

Therefore, in this RCT we studied associations between acute biological stress reactivity and recovery, as well as contextual memory related to an experimental trauma, and resulting intrusion development in healthy males $(\mathrm{N}=63$, mean age $=22.3)$. We used an adjusted version of the trauma film paradigm, which was hypothesized to result in more reliable biological stress reactivity and intrusive symptoms than the original version. Participants were randomized to undergo the socially-evaluated cold pressor test (coldwater $\mathrm{n}=29$ ) or control condition (warm-water $\mathrm{n}=34$ ) immediately prior to a trauma film. Biological and subjective reactivity was measured several times throughout the experimental assessment. Furthermore, Cued Recall, Recognition and Sequential Recall memory about the trauma film was assessed. Participants reported the occurrence of intrusions and its associated vividness and distress for the seven following days.

Linear Mixed Models revealed significantly increased acute stress reactivity (salivary cortisol and alpha amylase, subjective mood, blood pressure) and more intrusions, associated vividness and distress during the following week in the cold-water condition, while acute film-related declarative but not contextual memory was decreased. Linear regression models revealed that higher cortisol and alpha amylase reactivity and recovery levels predicted increased intrusion development during the following week, specifically in the cold-water condition, although this did not survive multiple comparison corrections. Contrary to what was expected, experimental trauma-related contextual memory and baseline HPA-axis negative feedback sensitivity for glucocorticoids were not predictive of intrusion development.

Thus, our study validated an adjusted trauma film paradigm to be more ecologically-valid by addition of the seCPT prior to watching the trauma film. Using this adjusted trauma film paradigm, we found additional support for the role of individual differences in biological stress reactivity and recovery in intrusion vulnerability. Obtaining an increased understanding of these individual differences and other potential factors of influence, such as sex and developmental stage of PTSD, could facilitate future development of effective prevention strategies for PTSD.

\section{8}

Telomere shortening in blood leukocytes of patients with posttraumatic stress disorder

\author{
Diana Avetyan ${ }^{1,2}$, Roksana Zakharyan ${ }^{1,2}$, Arsen Arakelyan ${ }^{1,2}$ \\ ${ }^{1}$ Laboratory of Human Genomics and Immunomics, Institute Of \\ Molecular Biology NAS RA, Yerevan, Armenia, ${ }^{2}$ Institute \\ of Biomedicine and Pharmacy, Russian-Armenian University, \\ Yerevan, Armenia
}

Telomeres are protective fragments on the chromosome ends involved in maintaining genome stability, preventing chromosomal fusions, regulation of cell division. It was shown that telomere attrition rate is accelerated in age-related diseases as well as in response to physiological and psychosocial stress. The aim of this study was to evaluate relative leukocyte telomere length (LTL) in patients with post traumatic stress disorder (PTSD) as well as to investigate association of functional SNPs of telomerase TERC and TERT genes with LTL and PTSD. The relative LTL was measured by multiplex quantitative PCR method; genotyping of TERC rs12696304, TERT rs7726159 and rs2736100 was performed by PCR with sequence specific primers. Comparison of LTL in diseased and healthy subjects showed that PTSD patients had shorter average LTL than controls. Also, the frequency and the carriers of the TERT rs2736100*T allele was higher in PTSD patients compared to controls. Overall, our results are in line with previous research in different populations. Furthermore, we demonstrated that rs 2736100 of TERT gene was significantly associated with PTSD and the minor allele of this polymorphism may be considered as a risk factor for PTSD in Armenian population.

\section{9}

\section{The diagnostic accuracy of the Turkish version of the General Practitioner Assessment of Cognition (GPCOG-Tr) in Turkish individuals}

\author{
Prof Dr Hakan Yaman ${ }^{1}$ \\ ${ }^{1}$ Independent Scholar, Antalya, Turkey
}

Background: To establish the diagnostic accuracy of the Turkish version of the General Practitioner Assessment of Cognition (GPCOG-Tr) in Turkish individuals. 
Materials and methods: The cross-sectional study was conducted in Antalya, Turkey, from February 2016 to April 2017. The purposive sampling method on of consecutively visited or attending participants was used in the study. MOCA Scale, GPCOG, and SMMT Scale were applied to the participants. Face validity was determined by two experts in this field. Construct validity was determined by factor analysis with principal component extraction method. Data were analyzed with descriptive analysis, internal consistency (Cronbach's alpha score), and ROC analysis. The level of significance was set at 0.05 .

Results: Three hundred one participants participated in this study (participation rate: $95 \%)$. Most of the participants were men $(n=183$, $60.8 \%)$ while $118(39.2 \%)$ were women. Their mean age were 71.02 years $(\mathrm{SD}=4.7$ years, $\min -\max =65-87, \mathrm{n}=301)$. Using The MOCA Scale as a reference (gold) standard, GPCOG showed accuracy (AUC $=0.774)$. Using The SMMT Scale as a reference (gold) standard, GPCOG showed accuracy (AUC $=0.752$ ). Cronbach's $\alpha$ coefficient was 0.515 for GPCOG scale. Intraclass Correlation for single measures and for average measures were 0.106 $(\mathrm{p}<0.001)$ and $0.515(\mathrm{p}<0.001)$, respectively. The area under the ROC curve (AUC) for GPCOG scale versus the MOCA Scale was 0.774 . A cut-off point of 6 or lover for GPCOG was applied and indicated the sensitivity of $84.4 \%$, and specificity of $55.8 \%$. The area under the ROC curve (AUC) for GPCOG scale versus the SMMT Scale was 0.752 . A cut-off point of 6 or lover for GPCOG was applied and indicated the sensitivity of $64.8 \%$, and specificity of $75.7 \%$.

Conclusion: The GPCOG-Tr is clinically well-suited for use in clinical practice.

\section{0}

\section{The role of HPA activity and sleep disturbances for mental health in adolescence}

\author{
Maren-jo Kater ${ }^{1}$, Anika Werner, Prof. Arnold Lohaus, Prof. \\ Angelika Schlarb \\ ${ }^{1}$ University Bielefeld, Bielefeld, Germany
}

Introduction: Adolescence is characterized as a time of increased stress experiences and sleep disorders. Previous studies showed that both difficulties are linked to mental health. However, these findings are mostly based on subjective methods. Therefore, in this study, we investigate if the hypothalamic-pituitary-adrenal axis (HPA) level and sleep disturbances are associated with mental health problems.

Method: 175 adolescents $(\mathrm{M}=14.3$ years, $\mathrm{SD}=1.8,55 \%$ female $)$ participated in the Trier Social Stress Test, where the HPA activity was captured by the salivary cortisol level at six time points. The area under the curve with respect to the ground (AUC-g) was calculated, indicating the overall cortisol response during the experimental situation. Additionally, the subjective stress level (Adolescent Stress Questionnaire), sleep disturbances (Sleep Disturbance Scale for Children), and psychological difficulties (DISYPS-III Screening) were assessed by questionnaires in the self-report. Two multiple hierarchical regressions tested if (a) the cortisol level and (b) the perceived stress level along with sleep disturbances predict mental health problems, while the models were adjusted for age and gender. Results: The results showed that both, the cortisol level as well as the subjective stress level, significantly predicted mental health problems. Sleep disturbances were also an independent significant predictor besides the stress components.

Conclusion: Adolescents experiencing increased stress levels, indicated by a high HPA activity and subjective stress levels, are at higher risk for having mental health problems. High psychological stress levels and poor sleep are thereby independent risk factors. This emphasizes the need to address coping-skills, relaxation, and sleep improving cognitive and behavior techniques in interventions for mental health in this age group.

\section{1}

\section{Understanding pathological fears and anxieties from a behavioural neuroscience perspective: implications for mechanisms of aetiology and change}

\section{Dr. Jan Richter ${ }^{1}$ \\ ${ }^{1}$ University of Greifswald, Greifswald, Germany}

Translating basic models of behavioural neuroscience to clinical applications, pathological fears (e.g. acute panic attacks) and anxieties (e.g. anxious apprehension) can be theoretically conceptualised as a cascade of dynamically changing defensive responses to stimuli mistakenly associated with threat. Guided by this trans-diagnostic model we investigated subjective (reported fear and panic symptoms), physiological (fear-potentiated startle, electrodermal activity, heart rate, and heart rate variability), and behavioural (passive and active avoidance) response patterns in about 500 patients with panic disorder with or without agoraphobia during an acute threat challenge. Results demonstrate complex interactions between the level of analysis and confirm central assumptions of the theoretical model including reactions patterns specific for pathological fears and anxieties. Here, panic attacks can be described as instances of a defence mode during imminent threat including strong sympathetic activation and flight behaviours. In contrast, anxious apprehension follows a pattern of attentive freezing behaviour under prefrontal control that is still partially in place but which, however, decreases with increasing proximity of perceived threat in favour of subcortical defensive activation. Additionally, we found genetic evidence that acute panic is associated with the serotonergic systems whereas anxious apprehension is related to genetic modulations within the hypothalamicpituitary-adrenal axis. Importantly, individual differences in fear responding prior therapy predicted differential outcomes of subsequent exposure based CBT treatment. Finally, we investigated the effects of therapy on changing response patterns in a laboratorycontrolled test-retest-design and found that reduction varied for different fear response systems suggesting different processes to may be involved in treatment effects. The presentation gives an overview of the translational model of a dynamic defensive processing in patients affected by fear and anxiety disorders and will discuss the implications for exposure based CBT. Here, the special role of fear extinction learning is pointed out and underpinned by current results form a recent study with over 700 patients with various anxiety disorders completing a pre-treatment extinction learning paradigm optimised for translational research and followed by a treatment protocol based on the principles of fear extinction learning. The results presented summarise some of the research activity within the German-wide research networks for anxiety disorders (panic-net and protect-AD) over the past 15 years. 
12

\section{A brief digital mindfulness-based intervention for patients with chronic obstructive pulmonary disease: protocol and preliminary results of a pilot randomized controlled trial}

\author{
BSc MSc Hannah Tschenentt ${ }^{1,2}$, Assoc.-Prof., M.D. Georg- \\ Christian Funk ${ }^{2,3}$, M.D. Florian Vafai-Tabrizi ${ }^{3}$, Univ.-Prof. Dr. Urs \\ Markus Nater ${ }^{1}$ \\ ${ }^{1}$ Department of Clinical and Health Psychology, Faculty \\ of Psychology, University of Vienna, Vienna, Austria, ${ }^{2}$ Karl \\ Landsteiner Institute for Lung Research and Pneumological \\ Oncology, Vienna, Austria, ${ }^{3}$ Klinik Ottakring, Vienna, Austria
}

Background: Patients with chronic obstructive pulmonary disease (COPD) do not only suffer from somatic symptoms but also from symptoms of anxiety and depression (defined as psychological distress) as well as stress. As pharmacological interventions showed only limited effectiveness in targeting the latter, a need for additional treatment options emerges. In other chronic conditions, mindfulnessbased interventions (MBIs) are effective in reducing symptoms of psychological distress and stress. Despite first promising results, research on MBIs in COPD patients is still scarce and mixed regarding their effects. Furthermore, it is crucial to implement mental health interventions adapted to the needs of COPD patients. Due to often experienced physical limitations and impaired mobility, digital MBIs seem particularly promising. Building on the above, this pilot randomized controlled trial aims to examine a) the feasibility of a brief digital MBI for COPD patients and b) its effects on psychological distress and stress.

Methods: 30 psychologically distressed (assessed by the Hospital Anxiety and Depression Scale, HADS-A $\geq 8$ or HADS-D $\geq 8$ ) COPD patients are screened for inclusion and exclusion criteria in a telephone interview and randomly assigned to the MBI (plus treatment as usual) or waitlist control group (treatment as usual). After a single face-to-face introductory session, patients in the MBI condition are instructed to conduct at least one of four brief audio-guided mindfulness-based exercises (10-15 min) daily for 8 weeks, delivered on their smartphones. Following an ecological momentary approach, psychological and respiratory variables (e.g. subjective stress, dyspnoea) are assessed before and after each exercise. These data will be analysed using multilevel modelling. Moreover, primary (psychological distress) and secondary outcomes (e.g. chronic stress, fatigue) are measured at baseline, 4 weeks, 8 weeks, and follow-up, including questionnaires and assessments of biological stress markers (hair cortisol, heart rate variability, electrodermal activity). The data will be analysed using repeated measures ANOVAs. Finally, in semistructured telephone exit interviews with 15 patients of the MBI group, the intervention's feasibility will be explored using a thematic analysis approach.

Results: The study protocol, progress and preliminary results will be ready for the congress.

Conclusion and implications: This is the first study examining the feasibility and effects of a brief digital MBI in COPD patients. The results could serve as basis for following large-scale clinical trials and promote the implementation of digital MBIs as add-on treatment in the clinical practice.
13 Forecasting PTSD prognosis from acute post-trauma
biomedical data: a machine learning multicenter cohort
study

dr Katharina Schultebraucks ${ }^{1}$, dr Marit Sijbrandij, dr Isaac GalatzerLevy, dr Joanne Mouthaan, dr Miranda Olff, dr. Mirjam van Zuiden

${ }^{1}$ Amsterdam UMC, location AMC, Dept of Psychiatry, Amsterdam, The Netherlands

Introduction: Posttraumatic Stress Disorder (PTSD) development is by definition preceded by exposure to traumatic events. Thereby, the early aftermath of traumatic events provides a unique opportunity for administering preventive interventions. These preventive interventions are thought to be more effective and feasible when they are targeted towards those individuals at highest risk of developing PTSD. Yet, current prognostic models for individual PTSD risk classification are not accurate enough for reliable implementation. This is likely because they do not sufficiently account for PTSD's heterogenic course and underlying complex etiology.

Aim: To overcome these limitations and reliably forecast long-term PTSD course, machine learning was applied on a broad range of biomedical data collected within $48 \mathrm{~h}$ post-trauma. We used a multinominal approach including the full spectrum of common PTSD symptom courses within one prognostic model for the first time (Schultebraucks et al. 2021 Neurobiology of Stress). We included biomedical data with the potential to be routinely collected in the ED, as this would enable time-efficient and inexpensive individual risk classification.

Methods: The data were collected in the framework of a larger prospective longitudinal cohort study on incidence and prediction of trauma-related psychopathology (Trauma TIPS, Mouthaan et al., 2013; Mouthaan et al., 2014a; Mouthaan et al., 2014b; Mouthaan et al., 2015). Here we included $\mathrm{N}=417$ patients (37.2\% females; mean age $46.09 \pm 15.88$ ) admitted with (suspected) serious injury to two urban Academic Level-1 Trauma Centers. Routinely collected biomedical information (endocrine measures, physiological (vital) measures, pharmacotherapy, demographics, injury and trauma characteristics) upon Emergency Department admission and within the subsequent $48 \mathrm{~h}$ were included as predictive features. Cross-validated multi-nominal classification of longitudinal self-reported symptom severity (IES-R) over 12 months and bimodal classification of clinician-rated PTSD diagnosis (CAPS-IV) at 12 months post-trauma was performed using extreme Gradient Boosting and evaluated on holdout sets. To obtain potential correlates of PTSD pathogenesis, we applied interpretable machine learning (SHapley Additive exPlanations (SHAP) values) to interpret the derived models and examine the complex associations between the most relevant biomedical variables and PTSD outcome.

Results: We achieved good prediction of PTSD symptom trajectories over 12 months (multiclass AUC $=0.89$ ) and end-point PTSD diagnostic status $($ AUC $=0.89)$. The 15 most relevant prognostic features to forecast both multinominal and bimodal PTSD outcome largely overlapped ( $66.7 \%$ overlap). These most relevant features included acute endocrine and physiological measures upon admission and hospital-prescribed pharmacotherapy within the first $48 \mathrm{~h}$ post-admission. For all continuous most relevant features, the decision rules for prognostic classification of bimodal PTSD outcome were nonlinear, emphasizing the complex etiology.

Conclusion: Individual risk for long-term PTSD was accurately forecasted from biomedical information routinely collected within $48 \mathrm{~h}$ post-trauma. By enabling more reliable PTSD risk classification early post-trauma, our results may facilitate future targeted preventive 
interventions. Additionally, our findings provide further insights into the complex etiology underlying PTSD and its heterogenic course.

\section{4 \\ Daily quarantunes: how music listening regulates stress and mood during COVID-19 lockdown}

\author{
Anja Feneberg ${ }^{1}, \mathrm{PhD}$ Paul Forbes ${ }^{1}, \mathrm{PhD}$ Claus Lamm ${ }^{1}$, Giulio \\ Piperno $^{1}$, Ekaterina Pronizius ${ }^{1}, \mathrm{PhD}$ Giorgia Silani ${ }^{1}$, Ana Stijovic ${ }^{1}$, \\ PhD Urs Nater ${ }^{1}$ \\ ${ }^{1}$ University of Vienna, Vienna, Austria
}

Introduction: Preliminary research has indicated that the COVID-19 pandemic led to an increase in music consumption with the specific purpose of stress and mood regulation. However, to date, ecologically valid research on the health-beneficial effects of music listening during the pandemic is lacking. Moreover, only little is known regarding factors moderating these effects in everyday life. The aim of this ecological momentary assessment study was to investigate the regulatory effects of music listening on perceived stress and mood in everyday life during the first wave of the COVID-19 pandemic. In addition, the modulating role of chronic stress and qualitative aspects of the music listening situation were examined.

Methods: Data collection took place between April, 1st, to Mai, 8th, 2020 in Austria and Italy, when strict self-isolation measures were established in both countries. The final sample comprised 711 adults (Mage $=31.6 \pm 11.6$ years, $69.9 \%$ female, $n=484$ residing in Austria, $\mathrm{n}=227$ residing in Italy). Participants rated their momentary perceived stress and mood levels (valence, calmness, energy) five times per day across seven consecutive days. Furthermore, participants reported whether they had been listening to music since the previous data entry at each assessment and, in case of confirmation, indicated perceived music characteristics and reasons for music listening. Chronic stress was assessed at the end of the study period. In order to unravel the dynamic regulatory properties of music listening, multilevel models were computed taking initial levels of stress and mood as well as covariates (e.g., time of day, country of residence) into account.

Results: Overall, music listening predicted lower subsequent perceived stress $(\mathrm{p}=.044)$ and enhanced mood levels $(\mathrm{ps}<.001)$. Specific interaction effects emerged for perceived stress and energetic arousal (ps $<.001$ ), with music listening predicting a downregulation of stress and energy most notably when initial levels were above average, and an upregulation of perceived stress and energy when initial levels were below average. In addition, individuals reporting higher chronic stress showed enhanced mood valence and energy after music listening ( $\mathrm{ps} \leq .01$ ). Music characteristics and reasons for music listening showed differential associations to stress and mood on the within- and between person level.

Conclusions: Findings from the present study suggest that music listening can be considered a powerful means for the regulation of stress and mood during psychologically taxing times, with particular benefits for those experiencing heightened levels of momentary and chronic stress. Daily life interventions incorporating music for stress and mood management should consider the dynamic interplay between characteristics of the music, the situation, and the listener.
15

Perceived social isolation is associated with the cortisol awakening response on workdays and free days in teachers

Sandra Isabell Schneider ${ }^{1,2}$, Alexander Wettstein ${ }^{2}$, Gabriel Jenni ${ }^{2}$, Fabienne Kühne ${ }^{2}$, Martin grosse Holtforth ${ }^{1}$, Roberto La Marca ${ }^{3,4}$

${ }^{1}$ University of Bern, Bern, Switzerland, ${ }^{2}$ University of Teacher Education, Bern, Switzerland, ${ }^{3}$ University of Zurich, Zurich, Switzerland, ${ }^{4}$ Clinica Holistica Engiadina, Susch, Switzerland

Objectives: Perceived social isolation (PSI), which can be defined as the perception of a lack of quality or quantity in social relationships, has been linked to numerous psychological and physical health problems, as well as physiological stress variables. The cortisol awakening response (CAR), i.e., an increase of cortisol levels after awakening, appears to be a valuable variable for psychoneuroendocrinological research, indicating psychosocial and physical health parameters. In the present study, we explore the hypothesis that PSI is associated with CAR in teachers on workdays and free days.

Methods: In an ambulatory assessment design, CAR was calculated as the area under the curve with respect to increase from three saliva samples (awakening, $+30 \mathrm{~min},+45 \mathrm{~min}$ ), which were collected on two workdays and one free day in 42 teachers ( 28 women; mean age $=39.66, \mathrm{SD}=11.99$ ). PSI was assessed with the Trier Inventory for Chronic Stress (TICS).

Results: CAR was significantly higher on the averaged workdays than on the free day $(\mathrm{p}<.001)$. PSI correlated significantly with CAR on the averaged workdays as well as the free day (both $\mathrm{p}<.05$ ).

Conclusion: The results suggest differences in morning cortisol levels between workdays and free days in teachers. In addition, perceived social isolation was positively associated with morning cortisol levels, supporting the link between perceived social isolation and physiological stress variables. Future studies should consider the impact of social characteristics (at work as well as in private life) when examining teacher stress.

Keywords: Cortisol Awakening Response, Perceived Social Isolation, Ambulatory Assessment, Stress

\section{6}

Impact of chronic ethnic discrimination in the daily life of Turkish immigrants living in austria: 30-day ambulatory assessment study

\author{
Mr. Andreas Goreis ${ }^{1}$, Dr. Urs Nater ${ }^{1}$, Dr. Dr. Ricarda Mewes ${ }^{1}$ \\ ${ }^{1}$ University Of Vienna, Vienna, Austria
}

Objective: Chronic ethnic discrimination is associated with negative mental and physical health outcomes in ethnic minority groups. It is assumed that suffering from repeated discriminatory events leads, over time, to psychological consequences such as higher perceived stress, higher negative affect, and lower positive affect. Higher stress reactivity to non-discriminatory stressors, such as daily hassles, as well as anticipation and avoidance behavior regarding discriminatory events, may further contribute to the overall burden for affected individuals. We investigated the impact of chronic ethnic discrimination and discriminatory events in the daily lives of Turkish immigrants living in Austria, using an ambulatory assessment design. Methods: $\mathrm{N}=90$ male Turkish immigrants $(\mathrm{n}=60$ who experienced chronic discrimination and $\mathrm{n}=30$ who experienced rare discrimination) were equipped with iPods and reported discriminatory events for 30 days. Perceived stress, perceived discrimination, negative and 
positive affect, stress reactivity to daily hassles, anticipation and avoidance behavior, as well as rumination with regard to discriminatory events were assessed each evening. Multilevel analyses were conducted.

Results: In preliminary analyses of $n=16$ participants, a total of 27 discriminatory events were reported. Event-based analyses showed that on days when discriminatory events occurred, participants in both groups reported a higher reactivity to daily hassles $(b=4.10$, $\mathrm{p}=.046)$ and higher avoidance behavior $(\mathrm{b}=1.78, \mathrm{p}=.001)$ than on days when no events occurred. On a descriptive level, the chronic ethnic discrimination group reported higher levels of stress $(b=0.53$, $\mathrm{p}=.240)$, more negative affect $(\mathrm{b}=3.72, \mathrm{p}=.143)$, a higher stress reactivity to daily hassles $(b=8.05, p=.119)$, and more anticipation $(\mathrm{b}=0.86, \mathrm{p}=.133)$ and avoidance behavior $(\mathrm{b}=0.70, \mathrm{p}=.158)$ than the rare ethnic discrimination group. Detailed between-group analyses and time-lagged models will be reported upon completion. Conclusion: The assessment of this first group of participants demonstrated that our ambulatory assessment design is feasible to investigate the effects of ethnic discrimination in the daily life of ethnic minority groups. Specifically, chronic ethnic discrimination was associated with negative psychological consequences in daily life. Investigations of the direct and indirect psychological consequences of discriminatory events in daily life are important and may promote the understanding of the link between chronic ethnic discrimination and health.

\section{7}

\section{Patient stratification based on pupillometric response profiles and its brain correlates in affective and anxiety disorders}

Julia Fietz $^{1,2}$, Dorothee Pöhlchen ${ }^{1,2}$, BeCOME working group ${ }^{1,3}$, Tanja M. Brückl ${ }^{1}$, Frank Padberg ${ }^{4}$, Elisabeth B. Binder ${ }^{1}$, Michael Czisch $^{3}$, Philipp Sämann ${ }^{3}$, Victor I. Spoormaker ${ }^{1}$

${ }^{I}$ Department of Translational Research in Psychiatry, Max Planck Institute of Psychiatry, Munich, Germany, ${ }^{2}$ International Max Planck Research School for Translational Psychiatry (IMPRS-TP), Max Planck Institute of Psychiatry, Munich, Germany, ${ }^{3}$ Max Planck Institute of Psychiatry, Munich, Germany, ${ }^{4}$ Department of Psychiatry and Psychotherapy, University Hospital LMU, Munich, Germany

In previous work, we showed that pupil size increases with increasing working memory load and is reliably correlated to activity in the frontoparietal network (FPN). Here, we investigate whether pupillometry constitutes a valid marker for biological patient stratification in a dimensional cross-diagnostic approach.

Our sample consisted of 123 psychiatric patients with affective and anxiety disorders (Mage $=35.02$ years, 86 female) who completed an N-back task with four conditions (fixation, 0-back, 1-back, 2-back) during functional magnetic resonance imaging (fMRI) and simultaneous pupillometry. We used latent class growth modeling (LCGM) to detect data driven subgroups with different pupillometric response profiles. The model with the best fit according to the Bayesian Information Criterion was chosen and the corresponding mean pupil values were entered into general linear models in SPM12. For second level group comparisons, we used propensity score matching to control for age and sex effects. We examined phenotypic group differences in depression (BDI-II), state and trait anxiety (SAI and TAI) and childhood trauma (CTQ).

The LCGM resulted in two distinct subgroups: one with a stepwise increasing pupil size with increasing working memory load $(n=97$, 'typical' group), and the other with a divergent pattern that was less reactive $(n=26)$. The statistical maps of the mean pupil values per block were largely similar showing mainly the FPN; the differential contrast indicated that the pupil response profile of the typical group revealed more activity in bilateral caudate, nucleus accumbens, temporoparietal junction as well as amygdala, posterior insula, and anterior cingulate gyrus. Additionally, the typical group showed higher scores on the CTQ $(\mathrm{t}(54)=1.7, \mathrm{p}=.008, \mathrm{~d}=-0.7)$, whereas no differences were observed for BDI-II, SAI, and TAI.

Through examination of individual pupil size growth patterns, we were able to identify potentially meaningful transdiagnostic latent subgroups possibly pointing towards an association between physiological signatures and childhood adversities.

\section{8}

\section{Psychobiological effects of chronic ethnic discrimination in Turkish immigrants: stress responses to standardized face-to-face discrimination in the laboratory}

\author{
Mr Andreas Goreis ${ }^{1}$, Dr. Urs Nater ${ }^{1}$, Dr. Dr. Ricarda Mewes ${ }^{1}$ \\ ${ }^{1}$ University of Vienna, Vienna, Austria
}

Background: Ethnic discrimination has a negative impact on the mental and physical health of ethnic minority groups. It has been proposed that the experience of stress and the concomitant dysregulation of stress-reactive biological systems, such as the autonomic nervous system (ANS) or the hypothalamus-pituitary axis (HPA axis), might act as an intermediary in this association. Previous studies have indicated that ongoing, "chronic", ethnic discrimination was associated with alterations of the ANS. Furthermore, indicators of dysregulation in the HPA axis, i.e., flatter diurnal cortisol slopes and higher hair cortisol concentrations, have been reported in ethnic minority samples. Despite these promising findings, no study has yet examined whether and how chronic ethnic discrimination influences the psychobiological reactions to an acute discriminatory event, i.e., when it actually happens. To address this research question, we studied acute psychobiological stress responses to a standardized discriminatory event in the laboratory in participants reporting either chronic or rare ethnic discrimination. We investigated male Turkish immigrants as an index group, as they constitute the largest group of non-EU citizens in several EU countries and are often the target of discrimination.

Methods: Seventy-two male Turkish immigrants were recruited: 35 participants who experienced chronic ethnic discrimination (CED) and 37 who experienced rare ethnic discrimination (RED), as classified by the Everyday Discrimination Scale (no group differences regarding age, BMI, and in-group identification; ps $>.536$ ). They were invited to the laboratory for a two-hour appointment and underwent a previously validated 10-min ethnic discrimination paradigm, i.e., a simulated physician's consultation with verbal and non-verbal discriminatory cues. Perceived stress, perceived discrimination, salivary cortisol (as a measure of HPA axis activity) and alpha-amylase (as a measure of ANS activity) were assessed seven times over the course of the appointment. Complementary ANS measures (heart rate, heart rate variability, and electrodermal activity) were measured continuously. Hair samples were collected for later determination of long-term cortisol concentrations. We analyzed stress responses via multilevel modeling.

Results: The ethnic discrimination paradigm elicited stress responses across all outcomes in both groups (main effects of time: ps $<.001$ ). The CED group reported higher levels of perceived stress (time $\times$ group $\mathrm{F}=10.846, \mathrm{p}<.001$ ) and discrimination (time $\times$ group: $\mathrm{F}=6.513, \mathrm{p}<.001)$ than the RED group in response to the ethnic discrimination paradigm. In addition, the CED showed flatter cortisol 
reactivity slopes in response to the ethnic discrimination paradigm (reactivity $\times$ group: $\mathrm{b}=-0.009, \mathrm{p}=.001$ ). No group differences were found regarding heart rate, heart rate variability, electrodermal activity, and alpha-amylase $(\mathrm{ps}>.05)$. Analyses of hair cortisol concentrations will be reported upon completion.

Conclusions: Participants experiencing CED appraised the standardized discriminatory situation as more stressful and more discriminatory than the RED group and showed a lower cortisol response. The combination of higher subjective stress with a flatter cortisol response may indicate a dysregulation of the HPA axis, which may be a consequence of CED. The investigation of the psychobiological effects of CED is crucial, as alterations in stress reactive systems may, over time, lead to illness and, eventually, to substantial health inequities in ethnic minorities.

\section{9}

\section{Enhanced motivation and facial reactions to interpersonal touch under stress following morphine administration}

\author{
Claudia Massaccesi ${ }^{1}$, Matthäus Willeit ${ }^{2}$, Boris Quednow ${ }^{3}$, Urs Nater ${ }^{1}$, \\ Giorgia Silani $^{1}$ \\ ${ }^{1}$ University of Vienna, Vienna, Austria, ${ }^{2}$ Medical University \\ of Vienna, Vienna, Austria, ${ }^{3}$ University of Zurich, Zurich, Switzerland
}

Introduction: Physical social contact, such as grooming in primates or touch in humans, is fundamental to create and maintain social bonds. The Brain Opioid Theory of Social Attachment postulates that $\mu$-opioids play a central role in social connection. Accordingly, pharmacological studies in isolated animals indicate that $\mu$-opioid agonists reduce, and $\mu$-opioid antagonists increase distress responses and motivation for social contact. Despite the abundance of animal studies, human evidence is still lacking.

Methods: Here, we investigated the neurochemical basis of social motivation and social pleasure under stress in healthy human volunteers $(\mathrm{N}=80)$, following either $10 \mathrm{mg}$ morphine ( $\mu$-opioid agonist) or placebo administration. After undergoing a well-validated stress induction procedure, participants were administered with gentle skinto-skin caresses on the forearm, at different speeds $(6,21$ and $27 \mathrm{~cm} /$ s). By adopting a translational approach, real physical effort (measured via a hand dynamometer) and facial hedonic reactions (measured via facial electromyography), together with self-reports of wanting and liking for interpersonal touch, were assessed. For data analysis, linear mixed effects models (LMM) were employed. Results from LMMs were controlled for the false discovery rate (FDR) associated with multiple testing using the Benjamini-Hochberg method.

Results: Preliminary results revealed cortisol suppression $(\mathrm{p}<.001)$ and greater negative subjective responses $(\mathrm{p}=.03)$ to stress following morphine administration, compared to the placebo group. This enhanced stress response led to increased subjective wanting of the most pleasurable touch $(\mathrm{p}<.01)$. Furthermore, the increased motivation for interpersonal touch in the morphine group was accompanied by greater activity of the corrugator muscle (CS) during reward anticipation $(\mathrm{p}=.04)$. CS activity was generally positively associated to subjective wanting in both groups $(\mathrm{p}=.04)$, possibly tracking enhanced attention toward the rewarding stimuli.

Discussion: The findings indicate that the morphine-induced block of cortisol possibly causes stronger negative emotions after stress induction. In line with animal models and previous evidence in humans, the observed negative reaction acts as a negative reinforcer on reward related behaviours, leading to increased wanting and facial reactions to the announced interpersonal touch.

\section{0}

\section{Acceptance of exposure therapy in VR vs. in vivo in an inpatient study sample}

Magdalena Sich ${ }^{1}$, Dr. phil. Julia Diemer ${ }^{1}$, Ines Sam ${ }^{1}$, Prof. Dr.,med. Peter Zwanzger ${ }^{1}$

${ }^{1}$ Kbo-inn-salzach-klinikum Wasserburg Am Inn, Wasserburg am Inn, Germany

Despite the availability of effective treatments for anxiety disorders, the treatment rate is alarmingly low (Wittchen et al. 2010; Alonso et al. 2018). One benefit of virtual reality exposure therapy (VRET) might be greater acceptance among patients. So, VRET could have the potential to at least partially fill this coverage gap (Opris et al. 2012). Surprisingly, there is only one study on this topic to date. García-Palacios et al. (2007) found a clear preference of VRET over exposure therapy in vivo. However, this study has methodological shortcomings worth discussing. All patients participated in outpatient clinical trials on the efficacy of VRET after the survey (GarciaPalacios 2007). Therefore, the validity of these results might be affected by a selection bias.

Despite $10 \%$ of patients with anxiety disorders undergoing inpatient treatment (Wittchen et al. 2010), there are no studies on differences in preference for either modality (VR vs. in vivo) in an inpatient setting. Therefore, the present study on VR acceptance investigated the expectations of inpatients within the framework of standard care at a large psychiatric hospital.

A total of $n=62$ patients took part in this randomized study. Patients with a manifest anxiety disorder or relevant fears (in the context of a different diagnosis) in inpatient or day-clinic treatment at our hospital were included. The main outcome variable was willingness to perform exposure in VR vs. in vivo. Secondary outcome measures were patients' expectations towards exposure therapy in VR vs. in vivo and their preference VR vs. in vivo.

All patients were shown a video sample of an exposure exercise that was relevant to their own fear (fear rating $>30 \%$ ). After that they completed a questionnaire to elicit expectations toward exposure exercises in the respective modality (VR or in vivo). This was followed by the second example video of the same exposure situation in the other modality (VR or in vivo) and filling in the accompanying questionnaire (VR or in vivo). Only then the experimenter inquired about the preference for either modality (VR vs. in vivo), as well as the reasons for their choice. The same procedure was repeated after the patient took part in a VR exercise (without fear cues), which was part of a different study. This procedure was chosen to test it first hand experience of VR influenced acceptance and/ or expectations.

To our knowledge, this is the first investigation after 2007, and the first to also control for effects of farmiliarity with VR, to examine patient preference for exposure therapy in VR vs. in vivo. Considering that patient treatment preferences affect dropout rates and response to therapy (Steidtmann et al. 2012), we conclude that VR has the potential to at least partially fill this coverage gap.

21

The association between repetitive negative thinking and distress across mental disorders in an outpatient treatment-seeking sample

Prof Dr Sebastian Trautmann ${ }^{1}$, Prof Dr Markus Muehlhan ${ }^{1}$, Dr Sandra Miethe ${ }^{1}$

${ }^{1}$ Medical School Hamburg, Hamburg, Germany 
Background: Distress is a characteristic of various mental disorders. The transdiagnostic construct repetitive negative thinking (RNT) has been suggested to contribute to distress. However, there is little evidence that this association can actually be found across diagnostic categories. This study aimed at (1) investigating the association between distress and RNT across different diagnostic categories and (2) examine whether distress is specifically associated with RNT over and above the contribution of general emotion regulation abilities.

Methods: The sample consisted of 150 female and 78 male treatment-seeking individuals which presented themselves at a German outpatient university clinic. To be included in the study, patients had to meet the criteria of at least one of the following diagnostic categories: phobias (specific phobia, agoraphobia, social phobia), other anxiety disorders (generalized anxiety disorder, panic disorder), stress disorders (acute stress disorder, posttraumatic stress disorder, adjustment disorder), depressive disorders (depressive episode, recurrent depressive disorder), substance use disorders (harmful use or dependence with regard to any psychoactive substance). Diagnostic status was established by clinical assessments by licensed psychotherapists based on ICD-10 criteria. Distress was measured using the general distress score of the Brief Symptom Inventory (BSI). RNT was assessed using the 15-item Perseverative Thinking Questionnaire (PTQ) which measures content-independent RNT. The 27-item selfreport measure of emotional competencies (SEK-27) was used to assess general emotion regulation abilities. Associations between distress and repetitive negative thinking were examined using correlation analyses. Partial correlations were further conducted to analyze the specific association between distress and repetitive negative thinking over and above the contribution of general emotion regulation abilities (SEK-27 scores).

Results: Twenty-four patients $(10.5 \%)$ met the criteria for any phobia, $28(12.3 \%)$ for any other anxiety disorder, $67(29.4 \%)$ for any stress disorder, $166(72.8 \%)$ for any depressive disorder and 14 $(6.1 \%)$ for any substance use disorder. Average distress T scores were markedly above the reference value of 50 for all diagnostic categories. Distress was associated with RNT across all examined diagnostic categories (zero-order correlations between .60 and .80). When accounting for general emotion regulation abilities in partial correlations, associations were similar or only marginally reduced with the exception of phobias where distress was no longer associated with RNT.

Discussion: As hypothesized, RNT was associated with distress across all diagnostic categories with high effect sizes. The association between distress and RNT was not attributable to general emotion regulation abilities except for phobias. Targeting RNT might be a highly promising transdiagostic approach to reduce distress in a variety of mental disorders. Longitudinal studies in larger samples using established diagnostic interviews to determine diagnostic status are needed to confirm these preliminary findings.

\section{2}

\section{Amygdala regulation using real-time fMRI based implicit closed-loop neurofeedback}

\author{
Ms. Apurva Watve ${ }^{1}$, Amelie Haugg ${ }^{2}$, Yury Koush ${ }^{3}$, David \\ Willinger $^{2}$, Annette Brühl ${ }^{1,4}$, Philipp Stämpfli ${ }^{5}$, Frank \\ Scharnowski $^{1,6}$, Ronald Sladky ${ }^{7}$ \\ ${ }^{1}$ Department of Psychiatry, Psychotherapy, and Psychosomatics, \\ Psychiatric Hospital, University of Zurich, Zurich, Switzerland, \\ ${ }^{2}$ Department of Child and Adolescent Psychiatry, Psychiatric \\ Hospital, University of Zurich, Zurich, Switzerland, ${ }^{3}$ Biomedical \\ Imaging Department, Yale University, United States, ${ }^{4}$ Center \\ for Affective, Stress and Sleep Disorders (ZASS), Psychiatric
}

University Hospital Basel, Basel, Switzerland, ${ }^{5}$ MR-Center of the Psychiatric University Hospital and the Department of Child and Adolescent Psychiatry, University of Zurich, Zurich, Switzerland, ${ }^{6}$ Department of Basic Psychological Research and Research Methods, Faculty of Psychology, University of Vienna, Vienna, Austria, ${ }^{7}$ Social, Cognitive \& Affective Neuroscience Unit, Faculty of Psychology, University of Vienna, Vienna, Austria

Introduction: Amygdala has a pivotal role in emotional regulation and exhibits functional connectivity with other brain regions involved in processing of emotional responses. The amygdala is particularly responsive to faces and, in this context, exhibits abnormal reactivity and connectivity in affective and anxiety disorders. It has been proven that down-regulation of amygdala as a form of voluntary emotional control can be achieved using real time fMRI neurofeedback (NFB) by implementing different explicit regulation strategies where subjects are aware about NFB training. Recent evidence suggests that successful regulation of the desired brain region is possible when subjects are oblivious about the training and the behavioral changes associated with it. Such an implicit approach could be equally effective to modulate brain activity and specifically advantageous when there are no explicit strategies available. To examine this concept, we applied a novel closed-loop NFB protocol in healthy participants where we presented facial stimuli with changing intensities of fearful and happy facial expressions based on the participant's amygdala activation i.e., the lower amygdala activity, the less fearful the face became and increase in amygdala activity increased the happiness of the facial stimuli.

Methods: Sixty four healthy participants performed four amygdala closed-loop NFB runs within one scan session. Thirty two subjects were assigned to the implicit group and were instructed to carefully observe the emotional faces, i.e. without receiving any information about the NFB setup. Thirty two subjects from the explicit group were informed about the NFB protocol and instructed to either reduce the fearful facial expression (fear-down) or increase the happiness of the presented face stimuli (happy-up). Functional MRI measurements were conducted on a $3 \mathrm{~T}$ Philips Achieva scanner and feedback was presented using Matlab based OpenNFT software. Each NFB run consisted of four 40 secs regulation blocks interleaved with four 20 secs baseline blocks.

Results and conclusion: Preliminary findings suggest that explicit and implicit groups showed a comparable change in amygdala activation in single NFB session. We observed that closed-loop NFB training induced a significant decrease in amygdala activation in response to fearful faces and a comparative increase in response to happy faces over four NFB runs suggesting subjects successfully learned to self-regulate their amygdala. This approach could open up new possibilities of NFB training in clinical conditions where patients have restricted cognitive ability to apply explicit regulation strategies.

\section{3}

\section{The effects of the COVID-19 pandemic on maternal mental health in postpartum}

Tanja Wolfgang ${ }^{1,2}$, Dr. med. Theresa Hübner ${ }^{2}$, Prof. Dr. med. Achim $\mathrm{W}$ öckel $^{2}$, Prof. Dr. med. Sarah Kittel-Schneider ${ }^{1}$, PD Dr. Catharina Bartmann $^{2}$

${ }^{1}$ Department of Psychiatry, Psychosomatic Medicine and Psychotherapy, University Hospital, Margarete-Höppel-Platz 1, 97080, Würzburg, Germany, ${ }^{2}$ Department of Obstetrics and Gynaecology, University Hospital, Josef-Schneider-Str. 4, 97080, Würzburg, Germany 
Background: Recent studies report an increase in anxiety and depression prevalence among pregnant and postpartum women during COVID-19 pandemic. The aim of the present study was to evaluate stress, depression and anxiety of pregnant and postpartum women during the first COVID-19-associated lockdown in Germany in spring 2020. Women who gave birth during the COVID-19 pandemic were assumed to be more affected by postnatal depression and anxiety than women who gave birth before the outbreak of the pandemic. Furthermore, the present study examined self-reported stress levels, maternal self-efficacy, mother-to-child bonding, COVID-19 specific anxiety as well as social isolation and sociodemographic variables.

Methods: As part of the prospective longitudinal study GeZeCO (Geburt in Zeiten von COVID-19) at the University Hospital of Würzburg, Germany (lead by PD Dr. med. Catharina Bartmann), pregnant and postpartum women were surveyed using standardized questionnaires at two measurement points during the COVID-19 pandemic: during the admission procedure in the delivery room (measurement point T1, April-June 2020) and 3-6 months postpartum (measurement point T2, September-November 2020). For the analysis of both time points, data of 62 female participants could be included. As a comparative sample, the data of 84 postpartum women could be included, which were collected by the working group of Prof. Dr. med. Sarah Kittel-Schneider prior to the outbreak of the pandemic.

Results: The results show that, contrary to what had been assumed, women who gave birth during the first months of the COVID-19 pandemic were not significantly more affected by depression and anxiety than the comparison sample. Similarly, no difference in mother-to-child bonding was evident between cohorts. However, it was found that subjects who had given birth during the COVID-19 pandemic had significantly lower maternal self-efficacy. In relation to the stress load of the experimental group, this was higher for multiple births than for first-time mothers, contrary to the assumption.

Conclusion: We could not show an increase in postnatal depression and anxiety in the first months of the COVID-19 pandemic in Germany. As a limitation, our study samples were rather small and differed in some sociodemographic variables. However, based on clinical experience and general data on depression and anxiety prevalence, we hypothesize that increased psychological distress might have been evident during the later months (third wave in Germany, end 2020/early 2021) of the COVID-19 pandemic due to increasing and longer restriction measures at this time also in mothers in the postpartum period.

\section{4}

\section{The influence of the covid-19 pandemic on relationship quality in consideration of the experienced stress and the dyadic coping}

\section{BSc Katharina Lehner ${ }^{1}$ \\ ${ }^{1}$ University of Vienna, Vienna, Austria}

Objective: Due to the covid-19 pandemic and its resulting restrictions such as curfews, changes in everyday work and uncertainties regarding the virus itself, many peoples' lives have been fundamentally altered. Therefore, these changes also impact intimate couples' relationships. The way of dealing with these new circumstances influences the extent to which stress affects relationships. Thus, the aim of this research is to examine the influence of the covid-19 restrictions on relationship quality, taking into account the intensity of the experienced stress and the level of dyadic coping.

Methods: A non-experimental cross-sectional study consisting of a sample of 370 participants currently living in a relationship, was conducted via an online questionnaire. The participants' average age was $27.6(\mathrm{SD}=7.7)$ years and consisted of 292 female, 74 male and 2 persons without specification of gender.

The ad-hoc questionnaire was created for this purpose and was composed of eight subscales. The subscales consisted of the variables relationship quality, external stress, stress due to covid-19, personal stressors, couple-internal stressors, dyadic coping, posttraumatic growth, resilience and an evaluation of the current life situation. Items and subscales of already existed questionnaires, such as the Covid Stress Scale (CSS, Taylor et al. 2020), the Pandemic Stress Index (Harkness et al. 2020) or the questionnaire to measure relationship quality (FPQ, Siffert and Bodenmann 2010), were used or adjusted for this study. For the assessment of personal stressors the General Health Questionnaire (GHQ-8, Goldberg et al. 1997) was applied, and for measuring Dyadic Coping a short version of the Dyadic Coping Inventory (DCI, Bodenmann 2008) was used.

Results: The results show that a majority reported an improvement or stagnation of their relationship quality during COVID-19. Moreover, regarding the extent of stress, couples with an enhanced relationship quality differed significantly from those whose relationship quality declined. Couples who experienced more stress also had a reduced relationship quality in comparison to couples with lower stress levels. A moderating effect of dyadic coping on the correlation between stress and relationship quality was found. Further analysis showed a mediating effect of couple-internal stress between external stress and relationship quality.

Conclusion: The results indicate that satisfied couples profit from a robustness against external influences. Additionally, many people reported that the value of the time spent together with their partner was much higher during the crisis.

As the results have shown, the degree of dyadic coping had a decisive impact on how much stress, caused by the crisis, affected the relationship in a negative way. It can be concluded from the results, that during the time of covid-19 external stress harmed relationships less than couple-internal stress. For analysis of the results, it should be taken into account that the sample consisted mainly of people with a high socioeconomic status. Nevertheless, many of them reported negative events during the covid-19-crisis, such as illness or death of a related person. To sum it all up, the study revealed that a functional romantic relationship can withstand stress in times of crisis without a decline in relationship quality.

\section{5}

\section{Neural oscillations in subsequent memory of associative fear learning}

Franziska Leimeister ${ }^{1}$, Ana Pesquita ${ }^{2}$, Ole Jensen ${ }^{2}$, Paul Pauli ${ }^{1}$, Julian Wiemer $^{1}$

${ }^{1}$ University of Würzburg, Würzburg, Germany, ${ }^{2}$ University of Birmingham, Birmingham, England

It is widely accepted that individuals with anxiety disorders show difficulties to suppress fear even in situations with the absence of threat. To better understand this phenomenon, it is crucial to understand the underlying cognitive mechanisms of safety learning. One possible way to investigate those underlying cognitive mechanisms is to study neural oscillation by recording electroencephalogram (EEG) while the formation of associative fear learning takes place. Former research that compared oscillatory power while the process of learning between items that were later remembered and items that were later forgotten could show that alpha, theta and gamma oscillations were predictive of successful memory retention. However, no 
study yet investigated how this effect might differ if the stimuli presented have a threat or safe character.

The experiment was divided into a learning, rating and re-exposure phase. In the learning phase participants were introduced to different faces which were either followed by electrical stimulation (US) or not and further were instructed to memorize those associations between faces (CS) and US or US omission. In a following memory test, participants were asked about their associative memory, arousal and valence regarding the previous seen stimuli. This memory rating allowed for later subsequent categorization of remembered and forgotten trials, with or without US occurrence. Finally, in the reexposure phase, all stimuli were presented once again and a fear rating was conducted to investigate if successful memory of safety influences the reported fear. We used the time-frequency approach to analyse alpha, theta and gamma power for the time period of stimulus onset in the learning phase. We therefore compared the oscillatory power between remembered trials that were followed by an US $(\mathrm{CS}+$ trials) and forgotten CS + trials, as well as remembered trials that were not followed by an US (CS - trials) and those CS - trials that were forgotten. Next to the measurement of neural oscillations we also investigated the role of pupil diameter and skin conductance responses in successful associative fear and safety learning throughout the study. Results will be presented at the conference.

\section{6}

\section{An investigation into the bi-directional association of stress and sexuality using an ecological momentary} assessment approach

\author{
MSc Hanna M. Müies ${ }^{1}$, PhD Charlotte Markert ${ }^{2}$, MSc Anja C. \\ Feneberg $^{1}$, PhD Urs M. Nater ${ }^{1}$ \\ ${ }^{1}$ University of Vienna, Vienna, Austria, ${ }^{2}$ Justus-Liebig-University \\ Gießen, Gießen, Germany
}

Background: Stress and sexuality substantially contribute to health and play an important role in daily life. The interaction between stress and sexual experience and behavior, however, has not yet been studied extensively. Some studies suggest a negative bi-directional association, while others provide contrasting evidence. The aim of this study is to investigate the bi-directional association between sexual experience and behavior, specifically sexual desire and arousal, and subjective stress in daily life. Hence, the study was designed as an ecological momentary assessment study providing high ecological validity and including event-based measurements following sexual activity.

Method: Fifty-eight heterosexual healthy individuals who had been in a relationship with their partner for at least one year participated in this study $(50 \%$ women, $\mathrm{M}=24.76$ years old, $\mathrm{SD}=2.96$, range: 19-31; 50\% men, $\mathrm{M}=24.28, \mathrm{SD}=3.09$, range: $20-32$ ). Following the baseline measurement, subjects completed data entries on a preprogrammed iPod seven times a day as well as after sexual activity for 14 consecutive days. Statistical analyses of nested data were conducted using multilevel modelling in HLM.

Results: Regarding gender differences in the main variables of interest, significant differences were found in sexual desire $(p=.005)$ and sexual arousal $(\mathrm{p}<.001)$, both of which were higher in men, but not in subjective stress levels $(\mathrm{p}=.799)$. Furthermore, in women, previous subjective stress levels significantly predicted sexual desire (UC $=-0.06, p=.029$, Pseudo $\left.R^{2}=0.217\right)$ and sexual arousal $\left(\mathrm{UC}=-0.05, \mathrm{p}=<.001\right.$, Pseudo $\left.\mathrm{R}^{2}=0.415\right)$ at the following measurement point. No such effect was found vice versa or in men. Discussion: While higher subjective stress levels predicted lower sexual desire and sexual arousal in women, no association between these variables was found in men. Hence, stress might constitute an important factor in women suffering from low sexual desire and arousal. These results should therefore be considered appropriately in a clinical context and might provide additional treatment options. The authors declare that there is no conflict of interest.

\section{7}

\section{Cortisol synchrony during the trier social stress test for groups or a non-stressful control task}

Bernadette Denk $^{1,2}$, Stephanie J Dimitroff ${ }^{1}$, Maria Meier ${ }^{1}$, Annika BE Benz ${ }^{1}$, Ulrike U Bentele ${ }^{1}$, Eva Unternaehrer ${ }^{1,3}$, Nathalie $\mathrm{F}$ Popovic $^{1}$, Wolfgang Gaissmaier ${ }^{1,2}$, Jens C Pruessner ${ }^{1,2}$

${ }^{1}$ University of Konstanz, Konstanz, Germany, ${ }^{2}$ Centre for the Advanced Study of Collective Behaviour, Konstanz, Germany, ${ }^{3}$ Department of Child and Adolescent Psychiatry, University of Basel, Psychiatric University Hospital, Basel, Switzerland

Physiological synchrony (PS) is defined as the co-occurrence and interdependence of physiological activity between interaction partners. Previous research has uncovered numerous influences on the extent of PS, such as relationship type or individual characteristics. Here, we investigate the influence of acute stress on PS. We do so in a setting in which PS was not promoted but contact between group members was explicitly minimized. We reanalyzed cortisol and subjective stress data from 138 participants (mean age $=23.48 \pm$ $3.99,47.1 \%$ female) who previously underwent the Trier Social Stress Test for groups (TSST-G) or a non-stressful control task together, collected as part of a larger project (Popovic et al. 2020). Using a stability and influence model, an established method to test for synchrony, we tested whether individuals' cortisol concentrations could be predicted by group members' cortisol levels. We found PS in participants who were in the same group, the extent of which was stronger in the non-stressful control condition. This suggests that while PS can occur in group settings even with spurious interaction, stressor exposure might attenuate its extent. We argue that if PS occurs in a sample where interaction was minimal, the phenomenon might be more widespread than previously thought. Further, stressor exposure might influence whether a situation allows for PS. We conclude that PS should be investigated within group settings with various degrees of social interaction to further expose mechanisms of and influence on PS.

\section{8}

How music gets under our skin: Preliminary results on the effects of music listening on stress and skin barrier recovery

Jasminka Majdandzic ${ }^{1,2}$, Prof. Elizabeth Broadbent ${ }^{3}$, Univ.-Prof. Dr. Urs M. Nater ${ }^{1,2}$

${ }^{I}$ Faculty of Psychology, University of Vienna, Vienna, Austria, ${ }^{2}$ Research Platform "The Stress of Life-Processes and Mechanisms underlying Everyday Life Stress", Vienna, Austria, ${ }^{3}$ Department of Psychological Medicine, University of Auckland, Auckland, New Zealand

Background: Psychological stress is known to have widespread effects on bodily health and immune function, as it is linked with dysregulation of the HPA axis. Music listening has been shown to be an effective method for alleviating stress, suggesting that music, by 
way of its effectiveness in reducing stress, may also have beneficial effects on immune function, for instance, speed of wound healing. In the present study, we assessed whether music listening after acute stress accelerates skin barrier recovery (SBR) after mechanical disruption, an established index of immune function, and whether this effect is mediated by reductions in stress.

Methods: We examined the effects of a 30-min music listening intervention on psychological and physiological indices of stress, and recovery of the skin barrier after impairment. Acute stress was induced in female participants using the Trier Social Stress Test, after which they were subjected to either a music listening intervention or one of two control conditions (audiobook, silence). A tape stripping paradigm was used to impair the skin barrier on participants' forearm, followed by repeated transepidermal water loss (TEWL) measurements over the course of the 4-h experiment to assess SBR. Subjective, physiological (heart rate, EDA), and salivary (alphaamylase, cortisol), stress markers were collected at multiple time points.

Results: Since the current sample of participants ( $\mathrm{n}=17$ for behavioural data, $\mathrm{n}=15$ for heart rate data, $\mathrm{n}=11$ for SBR data) is too small to conduct statistical analyses, data are reported descriptively in this abstract. Descriptive examination of our data provides some initial indication that music enhances Relaxed Positive Affect (known to be negatively correlated with stress) more strongly than an audiobook or silence. A similar pattern was observed for ratings on the scales Calm-Nervous and Good-Bad mood of the Multidimensional Mood State Questionnaire (MDBF). Likewise, subjective stress seemed to decrease more strongly after music listening than after listening to an audiobook or sitting in silence. In contrast, decreases in heart rate from peak stress to post-listening did not seem to differ between conditions. Finally, participants who listened to music seemed to show faster SBR than participants in the audiobook or silence conditions, as indicated by the area under the curve (AUC) aggregating all TEWL measurement time points after skin impairment.

Discussion: Descriptive examinations of our current sample offer some preliminary indications that a lab-based music intervention is effective in reducing stress and accelerating skin barrier recovery. Although findings from the current small sample should be interpreted with extreme caution, our paradigm is a promising tool for studying the effects of brief experimental manipulations on bodily health. Statistical analyses from a larger sample will be presented at the conference.

\section{9}

\section{Neural responses to acute stress predict chronic stress perception in daily life over 14 months: findings from the LawSTRESS project}

\author{
Marina Giglberger ${ }^{1}$, Hannah Luisa Peter ${ }^{1}$, Dr. Gina-Isabelle Henze ${ }^{1}$, \\ Sandra Zänkert ${ }^{1}$, Christoph Bärtt ${ }^{1}$, Julian Konzok ${ }^{1}$, Prof. Dr. Brigitte \\ Martina Kudielka ${ }^{1}$, Prof. Dr. Peter Kirsch ${ }^{2}$, Prof. (apl.) Dr. Stefan \\ Wüst ${ }^{1}$ \\ ${ }^{1}$ Institute of Psychology, University of Regensburg, Regensburg, \\ Germany, ${ }^{2}$ Department for Clinical Psychology, Central Institute \\ of Mental Health, University of Heidelberg, Medical Faculty \\ Mannheim, Mannheim, Germany
}

The objective of the prospective-longitudinal and quasi-experimental LawSTRESS project is to contribute to the understanding of the biopsychological mechanisms mediating the well-known link between chronic stress and the risk for several disorders.
In this project, 471 law students from Bavarian universities were studied over a 14-months period. The stress group (SG) consists of students experiencing a long-lasting and significant stress phase, namely the preparation for the final state examination for law students. Participants assigned to the comparison group (CG) were studied over an equally long period without particular stress exposure.

In the present analysis, we focused on the predictive value of neural responses to acute stress for stress perception in daily life over 14 months. Stress perception in daily life was measured on six sampling points (T1-T6) via the AA stress index, a newly developed 5 -item stress score with ten electronic queries on each sampling day. T1, twelve months prior to the exam, T2, three months pre exam, T5, one week post exam and T6, one month post exam, consisted of two consecutive sampling days, whereas at T3 and T4-both close to the exam-stress perception was measured only on one day. Additionally, the fMRI paradigm ScanSTRESS was applied to 123 students from the LawSTRESS sample at T1, including 60 subjects from the SG $(60 \%$ female, mean age $=22.73 \pm 1.58)$ and 63 subjects from the CG $(71.4 \%$ female, mean age $=21.10 \pm 2.05)$. ScanSTRESS consists of a two-run block design with stress and control conditions. It prompts the subject to solve arithmetic and rotation tasks while being evaluated by an observation panel. To test the association between AA stress index scores and neural responses in the a priori defined regions of interest amygdala, hippocampus and medial prefrontal cortex (mPFC), generalized linear multilevel models with the respective mean beta values as fixed effects were calculated.

Our analysis revealed a significant increase of the AA stress index in the SG until the exam followed by a distinct decrease, while the stress index in the CG stayed relatively stable. Remarkably, we found significant interactions between AA stress index increases and neural stress responses in amygdala $(\beta=-0.20, \mathrm{p}=.01)$, hippocampus $(\beta=-0.22, p=.02)$ as well as $\operatorname{mPFC}(\beta=0.13, p=.02)$ in the SG.

These findings document that the preparation for the final state examination was related to a significant increase of perceived stress. Furthermore, our results suggest that neural responses to acute psychosocial stress in amygdala, hippocampus and mPFC significantly predict stress perception in daily live during a long-lasting stress phase.

\section{0}

Neural responses to acute stress predict chronic stress perception in daily life over 14 months: findings from the LawSTRESS project

Marina Giglberger ${ }^{1}$, Hannah Luisa Peter $^{1}$, Dr. Gina-Isabelle Henze ${ }^{1}$, Sandra Zänkert ${ }^{1}$, Christoph Bärtl ${ }^{1}$, Julian Konzok ${ }^{1}$, Prof. Dr. Brigitte Martina Kudielka ${ }^{1}$, Prof. Dr. Peter Kirsch ${ }^{2}$, Prof. (apl.) Dr. Stefan Wüst ${ }^{1}$

${ }^{I}$ Institute of Psychology, University of Regensburg, Regensburg, Germany, ${ }^{2}$ Department for Clinical Psychology, Central Institute of Mental Health, University of Heidelberg, Medical Faculty Mannheim, Mannheim, Germany

The objective of the prospective-longitudinal and quasi-experimental LawSTRESS project is to contribute to the understanding of the biopsychological mechanisms mediating the well-known link between chronic stress and the risk for several disorders.

In this project, 471 law students from Bavarian universities were studied over a 14-months period. The stress group (SG) consists of students experiencing a long-lasting and significant stress phase, namely the preparation for the final state examination for law students. Participants assigned to the comparison group (CG) were studied over an equally long period without particular stress exposure. 
In the present analysis, we focused on the predictive value of neural responses to acute stress for stress perception in daily life over 14 months. Stress perception in daily life was measured on six sampling points (T1-T6) via the AA stress index, a newly developed 5 -item stress score with ten electronic queries on each sampling day. T1, twelve months prior to the exam, T2, 3 months pre exam, T5, 1 week post exam and T6, 1 month post exam, consisted of two consecutive sampling days, whereas at $\mathrm{T} 3$ and $\mathrm{T} 4$ - both close to the exam-stress perception was measured only on one day. Additionally, the fMRI paradigm ScanSTRESS was applied to 123 students from the LawSTRESS sample at T1, including 60 subjects from the SG $(60 \%$ female, mean age $=22.73 \pm 1.58)$ and 63 subjects from the CG $(71.4 \%$ female, mean age $=21.10 \pm 2.05)$. ScanSTRESS consists of a two-run block design with stress and control conditions. It prompts the subject to solve arithmetic and rotation tasks while being evaluated by an observation panel. To test the association between AA stress index scores and neural responses in the a priori defined regions of interest amygdala, hippocampus and medial prefrontal cortex (mPFC), generalized linear multilevel models with the respective mean beta values as fixed effects were calculated.

Our analysis revealed a significant increase of the AA stress index in the SG until the exam followed by a distinct decrease, while the stress index in the CG stayed relatively stable. Remarkably, we found significant interactions between AA stress index increases and neural stress responses in amygdala $(\beta=-0.20, \mathrm{p}=.01)$, hippocampus $(\beta=-0.22, p=.02)$ as well as $\operatorname{mPFC}(\beta=0.13, p=.02)$ in the SG.

These findings document that the preparation for the final state examination was related to a significant increase of perceived stress. Furthermore, our results suggest that neural responses to acute psychosocial stress in amygdala, hippocampus and mPFC significantly predict stress perception in daily live during a long-lasting stress phase.

\section{1}

\section{Threat belief determines the degree of costly safety behavior: assessing rule-based generalization of safety behavior with a dimensional measure of avoidance}

\author{
Dr. Alex Wong ${ }^{1}$, Prof. Dr. Andre Pittig ${ }^{2}$ \\ ${ }^{1}$ Julius-Maximilians-Universität Würzburg, Würzburg, Germany, \\ ${ }^{2}$ Friedrich-Alexander-Universität Erlangen-Nürnberg, Erlangen, \\ Germany
}

Excessive generalization of safety behavior to innocuous stimuli that resemble a feared stimulus is oftentimes maladaptive. Safety behavior is conventionally assessed dichotomously, requiring multiple presentations of each test stimulus and examines the proportion of safety behavior executed across all trials per stimulus. Thus, the generalization gradient confounds with ongoing extinction learning during non-reinforced test trials. The present study employed a recently developed dimensional measure of avoidance to examine the generalization of safety behavior, which can be seen as the extent of safety behavior engagement. Using a single-cue conditioning procedure, we found a range of distinct generalization gradients in safety behavior, which were highly consistent with participants' reported relational rules. Importantly, a dimensional measure of avoidance was able to examine the generalization gradients of safety behavior even when each test stimulus was presented once, thus minimizing the effect of ongoing extinction learning. In addition, the generalization rules parallel how clinically anxious individuals develop different threat beliefs after trauma exposure.
32

\section{The effect of median raphe region and its serotoninergic neurons on body temperature regulation in cold stress,} depressive-like behaviour and social behaviour

Csilla Lea Fazekas ${ }^{1,2}$, Manon Bellardie ${ }^{1,3}$, Bibiána Török ${ }^{1,2}, \mathrm{PhD}$ Eszter Sipos ${ }^{1}$, Mihály Dobos-Kovács ${ }^{1}$, Elodie Chaillou ${ }^{3}$, Dr. Dóra Zelena $^{1,4}$

${ }^{1}$ Institute of Experimental Medicine, Budapest, Hungary, ${ }^{2}$ János Szentágothai Doctoral School of Neurosciences, Semmelweis University, Budapest, Hungary, ${ }^{3}$ INRAE Centre Val de Loire, CNRS, IFCE, Université de Tours, UMR 85 Physiologie de la Reproduction et des Comportements, Tour, France, ${ }^{4}$ Centre for Neuroscience, Szentágothai Research Centre, Institute of Physiology, Medical School, University of Pécs, Pécs, Hungary

The midbrain median raphe region (MRR) has been linked to numerous different behaviours and stress adaptation, but its effect on vegetative functions is debated. Additionally, it is unknown if there is connection between them. The MRR is mostly known for its serotonergic $($ SERT +$)$ neurons, although they constitute a minor population of the nucleus. Our aim was to investigate the role of MRR, especially its SERT + neurons in different aspects of social and depressive-like behaviours in parallel with changes in core body temperature (BT) as a vegetative function upon cold stress.

Using pharmacogenetics control, excitatory and inhibitory designer receptors (DREADDs) were expressed in the mouse MRR. A biotelemetry system was implanted into the abdominal cavity to monitor changes in BT. Following injection of clozapine- $N$-oxide (CNO), the ligand for DREADDs, behavioural tests were performed. Social behaviour was investigated with sociability, social interaction (SIT) and resident intruder (RIT) tests. Depressive-like behaviour and cold stress reactivity was measured by the forced swim test (FST) at $23{ }^{\circ} \mathrm{C}$ water temperature. The same protocol was repeated in SERTCre mice, expressing the DREADD only in the SERT + cells of the MRR, but only with control and excitatory groups.

The manipulation of the MRR did not affect sociability. In the RIT the excitatory group showed increased friendly social behaviour. Additionally, in the FST, excitation increased floating, while marginally decreasing struggling. Only in the FST could we see a change in BT (cold exposure). The drop in BT was smaller after stimulating all neurones of the MRR, both during and after the test. In the SERTCre mice social behaviour was not affected in any of the tests. However, in the FST, excitation of MRR SERT + neurons marginally increased floating and significantly decreased struggling. Furthermore, the previously seen decrease in BT to FST after whole MRR stimulation was reproduced in the SERT-Cre excitatory group. Neither the stimulation of the whole MRR, nor its serotoninergic neurons affected plasma corticosterone and adrenocorticotropin releasing hormone levels.

While the MRR effectively regulates social and depressive-like behaviour as well as BT, SERT + neurons are only involved in part of these processes. Our results show that other populations of neurons might increase friendly social behaviour, while SERT + cells are responsible for the depressive-like behaviour, in parallel with decreased BT during cold exposure. This latter finding may have clinical relevance in human depressive disorders. 
33

\section{Differences in mental crises between children, adolescents, and their parents with and without migration background}

Arabella Bergmann ${ }^{1}$, Elina Mizgir ${ }^{1}$, Dr. Katharina Allgaier ${ }^{1}$, Dr. Daniela Hagmann ${ }^{1}$, Priska Schneider ${ }^{1}$, Hanna Brauner ${ }^{1}$, Dr. Gottfried Maria Barth $^{1}$, Dr. Jan Kühnhausen ${ }^{1}$, Prof. Dr. Annette Conzelmann ${ }^{1}$, Prof. Dr. Tobias Renner ${ }^{1}$

${ }^{1}$ Department of Child and Adolescent Psychiatry, Psychosomatics and Psychotherapy, University Hospital of Psychiatry and Psychotherapy, Tübingen, Germany

Objective: In recent years there has been a significant increase in emergency admissions at German child and adolescent psychiatries (CAP). Such emergencies are evoked by mental crises which are highly stress maintaining situations for all involved-especially children, adolescents and their parents. One subgroup of these emergencies are children and adolescents with migration background (MB). They face unique challenges throughout their development, compared to their native peers. Different factors have already been identified to explain differences in general mental health problems between children with and without MB (e.g. the process of migration, the ethnic minority position, the specific cultural background), but factors that are associated with mental crises are unknown so far. At the same time, almost $40 \%$ of all children and adolescents in Germany have MB. For these reasons we find the need to further examine how aspects of psychiatric emergencies regarding CAP differ within families with and without MB. Therefore, strain and psychological stress in both groups of emergencies - children and adolescents with and without MB as well as their parents-will be compared.

Method: In the ongoing study, data will be collected for one year until 1, July 2021. We aim to collect data of $\mathrm{N}=60$ children and adolescents with and without $\mathrm{MB}$ (each group $\mathrm{N}=30$; age maximum: $17 ; 11$ years) as well as their parents. The criteria for MB is met, if a child, an adolescent or at least one parent was born outside of Germany or born without the German citizenship. The questionnaires used for measuring the children's and adolescents stress level are YSR/11-18R (self-report) and CBCL/6-18R (parent report). These allow predications about internalizing and externalizing problems. The parental stress level will be assessed by using the EBI (German equivalent of the Parental Stress Index), which enables predictions about different stress maintaining areas of parenthood. The present study is part of an extensive research project that has been running at the CAP Tübingen since last year. This project aims to identify predisposing, provoking and maintaining factors for different indications at CAP Tübingen (planned outpatient, emergency outpatient, emergency inpatient and pupils) in order to improve and adapt psychological working methods.

Conclusion: With this research, we are planning to obtain substantial information about potential differences between emergency patients at CAP Tübingen with and without MB. By examining migration related specificities, we first intend to identify risk factors. Secondly, we intend to derive preventive and protective measures that allow a more specific and differentiated psychotherapeutic approach for mental crises which are adapted to the specific needs of children and adolescents with MB.

\section{4}

\section{Centralized gaze as a threat-specific component of defensive states in humans}

\author{
Alma-Sophia Merscher ${ }^{1}$, Prof. Dr. Matthias Gamer ${ }^{1}$ \\ ${ }^{1}$ University of Wuerzburg, Wuerzburg, Germany
}

Anxiety and trauma-related disorders place a huge burden on many individuals in our society. Exaggerated or persistent defensive reactions are thought to contribute to the development and maintenance of these disorders. However, it is still surprisingly unclear how to discern adaptive and dysfunctional responses to threat. This partly stems from conceptual confusion and methodological inconsistencies in the literature on defensive states and difficulties in translating results from animal studies to human participants and patients. In the current study, we aimed to increase conceptual clarity by characterizing the reliability and validity of individual components of defensive states in humans.

In animals, a particular defensive state upon real or anticipated threat consists of episodes of freezing accompanied by transient decreases in heart rate (bradycardia). This phenomenon has been interpreted as adaptive in that it helps to avoid predator detection and prepare fast action to approaching threat. Humans seem to show a similar defensive state that is paralleled by oculomotor inhibition. In a previous study, participants showed more centralized, fewer and longer fixations along with bradycardia and increases in skin conductance when anticipating an aversive stimulus that could be avoided by a fast button press.

The current two laboratory experiments inspected whether these action-preparatory responses are sensitive to demands in spatial attention and whether they depend on valence of the reinforcing stimulus. Subjects viewed naturalistic images while expecting a certain, no or a potential electrotactile stimulation (Experiment 1) or reward (Experiment 2) that could be avoided or gained by a fast joystick movement. In both experiments, eye movements (fixations), heart rate and skin conductance were recorded.

Experiment 1 replicated previously found centralized gaze, bradycardia, and skin conductance increases when subjects could avoid aversive stimulation. Increased centralization of gaze was related to shorter reaction times. Experiment 2 failed to find globally narrowed gaze behavior. In contrast, bradycardia and increases in skin conductance were also observed when participants could gain a reward by a quick movement.

While bradycardia and concomitant sympathetic activation reflect action-preparatory states independent of valence, centralization of gaze seems a robust and threat-specific phenomenon during the anticipation of avoidable threat. Thus, instead of relying on single readouts, translational research in animals and humans, should consider the multi-dimensionality of defensive states, especially when investigating ambivalent, conflicting situations. As a whole, the current experiments contribute to a more comprehensive and differentiated picture of defensive states, which seems pertinent to both animal and human research in the light of discrepancies in the previous literature. Its results might inspire future translational work in rodents and humans on shared mechanisms of threat processing, ultimately supporting the development of novel therapeutic approaches. 


\section{5}

\section{The potential impact of music listening style on pain and stress: an experimental approach}

Rosa M. Maidhof ${ }^{1}$, Alexandra Wuttke-Linnemann ${ }^{2,3}$, Mattes B. Kappert $^{4}$, Andreas Schwerdtfeger ${ }^{5}$, Gunter Kreutz ${ }^{6}$, Urs M. Nater ${ }^{1}$

${ }^{1}$ University of Vienna, Vienna, Austria, ${ }^{2}$ University Medical Hospital Mainz, Mainz, Germany, ${ }^{3}$ Center for Mental Health in Old Age, Landeskrankenhaus, Mainz, Germany, ${ }^{4}$ Philipps University of Marburg, Marburg, Germany, ${ }^{5}$ University of Graz, Graz, Austria, ${ }^{6}$ Carl von Ossietzky University of Oldenburg, Oldenburg, Germany

Objective: Music listening is an effective, easily applicable and inexpensive tool in pain and stress management. The processing of music is influenced by the music listening style. While music empathizers (ME) focus mainly on emotional aspects of music, music systemizers (MS) focus more on structural aspects of music. The role of the music listening style for pain and stress is widely unexplored. This study aimed to investigate whether pain and stress responses to acute pain differ in ME and MS. Men and women differ in their typical music listening styles: While men are more often MS than women, women are more often ME than men. We expected significant differences in pain and stress reactions between male and female $\mathrm{ME} / \mathrm{MS}$.

Methods: Four groups of healthy participants (female/male ME, female/male MS) listened to auditory stimuli during a cold pressor test (CPT) on three days each. On one day, researcher-selected music was presented. On the second day, participant-selected music was presented, and on the third day, the sound of lapping water was presented. The order of the three days was randomized. In order to measure pain responses, pain intensity and pain tolerance were repeatedly measured. For the investigation of stress-responsive systems, subjective acute stress, heart rate (HR), heart rate variability parameter RMSSD, salivary alpha-amylase (sAA) and salivary cortisol (sCort) were repeatedly measured. Data were analysed with multilevel analyses.

Results: Analyses $(\mathrm{N}=61$; age: $\mathrm{M}=24.2, \mathrm{SD}=3.89)$ showed that the CPT elicited pain and stress reactions in all investigated variables. The highest increase in pain intensity from a baseline measurement to the measurement directly after the CPT was found for ME compared to MS in participant-selected music in comparison to researcher-selected music and the sound of lapping water. This effect did not differ in men and women. Pain was tolerated for the longest time when participant-selected music was presented, but music listening style and gender did not modulate this effect. With regard to the stress parameters, there were interaction effects between music listening style, gender and time in HR and RMSSD, but not in subjective acute stress, sAA, or in sCort.

Conclusion: Our findings suggest that the music listening style differentially influences pain- and stress-related parameters. Music with high emotional relevance is associated with a high increase in pain intensity in ME, potentially due to an emotional overload. Music with less emotional relevance might be more beneficial for ME in reducing pain intensity. At the same time, pain tolerance is less influenced by music listening style and is highest when participant-selected music is used. The influence of music listening style and gender on parasympathetic activity might be stronger than on sympathetic and endocrine activity and on subjective acute stress. The study provides novel information on individual differences in coping with pain and stress.

\section{6}

\section{Comorbidity of anxiety and Alzheimer's disease in the triple transgenic mouse model}

\author{
Dorottya Várkonyi ${ }^{1,2}$, Dr. Eszter Sipos ${ }^{1}$, Bibiána Török ${ }^{1}$, Dr. Dóra \\ Zelena $^{1,3}$ \\ ${ }^{1}$ Institute of Experimental Medicine, Budapest, Hungary, ${ }^{2}$ Eötvös \\ Loránd University, Faculty of Science, Budapest, Hungary, ${ }^{3}$ Centre \\ for Neuroscience, Szentágothai Research Centre, Institute \\ of Physiology, Medical School, University of Pécs, Pécs, Hungary
}

Alzheimer's disease (AD) is a progressive neurodegenerative disorder, which is the most common cause of dementia in the elderly. Approximately $50 \%$ of $\mathrm{AD}$ patients develop depressive symptoms which can be triggered or aggravated by chronic stress. Anxiety also occurs in $\mathrm{AD}$ cases, contributing to cognitive decline. AD is considered as a major health issue which is still incurable, therefore appropriate animal models are needed for further preclinical studies.

The aim of the present study is to explore the appearance of anxiety-like symptoms in male triple-transgenic mouse model of AD (3xTg-AD, expresses human mutated PSEN1, APP and tau protein) before and after appearing plaque-like depositions (at the age of 4and 8-month-old) compared to age-matched controls. Cognitive decline was also tested to confirm if it is a relevant in vivo model of typical behavioural dysfunctions of AD. A test battery was used to assess anxiety-like behaviour consisting of open field (OF), elevated plus maze (EPM), light-dark box (LD) and social interaction (SI) tests. To examine cognitive decline, Morris Water Maze test (MWM) was applied.

During all tests AD mice moved significantly less than the controls therefore we were concentrating on locomotion-independent parameters. In the OF test, both age groups of $3 \times \mathrm{Tg}$-AD mice spent more time with self-grooming. Furthermore, $3 x \mathrm{Tg}$-AD spent less time with social interaction compared to control mice, but interestingly, this difference only appeared at 8 months of age in SI test. There was no significant difference in EPM and LD tests between control and 3xTg$\mathrm{AD}$ groups. In the MWM, the latency to find the platform was higher for 3xTg-AD mice compared to controls, both in 4-month and 8 -month-old cohorts.

We can conclude that $3 \times \mathrm{Tg}-\mathrm{AD}$ is a relevant in vivo mouse model of $\mathrm{AD}$ as memory decline can be observed in MWM. We confirmed the appearance of anxiety-like behaviour. However, only the SI test results showed significant progression in anxiety-like symptoms between the 4-months-old and the 8-months-old 3xTg-AD mice. All in all, our results provide further explanation about $3 \times \mathrm{Tg}-\mathrm{AD}$ mouse model and the comorbidity of $\mathrm{AD}$ and anxiety. This mouse model may be suitable for researching biomarkers that can cause these behavioural changes as well as for testing new treatment options for this comorbidity.

37

\section{Effects of social isolation on stress, mood, and motivation: evidence from laboratory and field studies}

MSc Ana Stijovic ${ }^{1}$, B.A. MSc PhD Paul Forbes ${ }^{1}$, Dr. Livia Tomova ${ }^{2}$, Dipl.-Psych. Dr. Nadine Skoluda ${ }^{1}$, BSc MSc Anja Feneberg ${ }^{1}$, Giulio Piperno $^{3}$, BSc MSc Ekaterina Pronizius ${ }^{1}$, Univ.-Prof. Dr. Urs Nater ${ }^{1}$, Univ.-Prof. Mag. Dr. Claus Lamm ${ }^{1}$, Assoz. Prof. Giorgia Silani ${ }^{1}$

${ }^{1}$ University Of Vienna, Vienna, Austria, ${ }^{2}$ University of Cambridge, United Kingdom, ${ }^{3}$ Sapienza University of Rome, Rome, Italy 
Introduction: Affiliative social contact is a fundamental need of social mammals. Even brief periods of social isolation were shown to induce an adaptive response in animals, including increased secretion of the stress hormone cortisol and increased motivation for social contact. From human research we know that long-term perceived social isolation predicts negative health outcomes. However, human adaptive responses to short-term objective social isolation are less understood. The aim of this study was to test the effects of experimentally induced acute social isolation on motivation, stress, and mood in humans and to assess the specificity of this deprived state against the more studied state of fasting. In order to test the ecological validity of the laboratory study, stress, mood and motivation during isolated and non-isolated days in the lab were compared to data gathered via ecological momentary assessment (EMA) in real life, during the first COVID-19 lockdown.

Methods: For the laboratory study, a repeated-measures experimental design was applied, in which 30 female participants came to the lab on three separate days: for a social isolation, a fasting and a baseline condition. Conditions were highly comparable, with the only difference being whether food and social contact were provided. Salivary cortisol and alpha-amylase were measured hourly, and heart rate was continuously measured throughout the session. Self-report measures assessing stress, mood, desire for social contact and food was collected every 1-2 h. For the field study, a subsample of individuals, participating in a large-scale EMA study during the first COVID-19 lockdown in Austria and Italy, and who reported at least one day without and one day with social contact, were selected and examined on the same self-report measures collected in the lab. The outcome measures were then compared between isolation and non-isolation days within-participant.

Results: Experimental induction of social isolation and fasting resulted in expected changes in self-reported motivation for the deprived stimulus, with need for social contact being higher in the isolation condition $(\mathrm{p}<0.001)$ and need for food higher in the fasting condition $(\mathrm{p}<0.001)$, compared to the baseline. Social isolation and fasting comparably led to lower reports of energetic arousal $(p<0.001$ and $p=0.003$, respectively) and higher fatigue $(p=0.004$ and $\mathrm{p}<0.001$, respectively), compared to baseline, as well as a higher desire to avoid the situation ( $\mathrm{p}=0.01$ and $\mathrm{p}=0.001$, respectively). The results from the EMA study partially replicated the results from the lab.

Conclusions: These preliminary findings indicate comparable modulation of energy, fatigue and self-reported motivation by social isolation and fasting in an experimental context, suggesting that acute social isolation in humans can be considered a deprived state with prompt affective and motivational adaptive responses. Furthermore, we will discuss similarities and discrepancies between experimental findings and findings from the context of forced social isolation during the COVID-19 lockdown.

\section{8 \\ Pharmacological vasopressin deficiency might influence maternal-separation-induced ultrasonic vocalization and stress-hormone levels in Wistar rat pups}

\author{
Bibiána Török ${ }^{1,2}$, M.D., PhD Anna Fodor ${ }^{1}$, PhD Sándor Zsebők ${ }^{3}$, \\ PhD Eszter Sipos ${ }^{1}$, M.D., PhD, DSc Dóra Zelena ${ }^{1,4}$ \\ ${ }^{1}$ Institute of Experimental Medicine, Budapest, Hungary, ${ }^{2} J a ́ n o s$ \\ Szentágothai School of Neurosciences, Semmelweis University, \\ Budapest, Hungary, ${ }^{3}$ Institute of Ecology and Botany, Centre \\ for Ecological Research, Vácrátót, Hungary, ${ }^{4}$ Centre
}

for Neuroscience, Szentágothai Research Centre, Institute of Physiology, Medical School, University of Pécs, Pécs, Hungary

Vasopressin can affect the appearance of anxiety in adulthood, but less is known about the perinatal period in this regard. Applying an appropriate animal model to explore the early postnatal age is essential. Previous studies have shown that rat pups emit ultrasonic vocalizations induced by maternal separation (MS-USV) as a sign of distress. Interestingly, in our previous results reduced MS-USV was reported in 7- to 8-day-old Brattleboro rat, which is genetically vasopressin-deficient because of a frameshift mutation in the gene coding this neuropeptide.

In this study our aim was to investigate the contributing vasopressin-receptor subtypes in this phenomenon.

7- to 8-day-old male and female Wistar rat pups were used. MSUSV was recorded for $10 \mathrm{~min} 30 \mathrm{~min}$ after intraperitoneal V1a-, V1b- or V2-receptor antagonist treatment (V1aR antagonist: SSR49059, V1bR antagonist: SSR149415, V2R antagonist: SSR121463B). Three different concentrations were applied to study dose-dependence in each type of antagonist treatment: 3,10 or $30 \mathrm{mg} /$ $\mathrm{kg}$. Sedation was studied by righting reflex and negative geotaxis, and finally, stress-hormone levels were measured by radioimmunoassay.

The genetically vasopressin-deficient Brattleboro pups showed decreased MS-USV even after a saline injection, accompanied by reduced adrenocorticotropin and unchanged corticosterone levels. In terms of the pharmacological vasopressin deficiency, $30 \mathrm{mg} / \mathrm{kg} \mathrm{V} 1 \mathrm{aR}$ antagonist increased corticosterone levels. All V1bR antagonist doses decreased MS-USV and adrenocorticotropin, while when we treated with $10-10 \mathrm{mg} / \mathrm{kg}$ of V1aR-V1bR antagonists, it decreased MS-USV without influencing stress-hormones. Three $\mathrm{mg} / \mathrm{kg}$ V2R antagonist enhanced MS-USV, while $30 \mathrm{mg} / \mathrm{kg}$ increased stress-hormone levels.

We confirmed that genetic vasopressin-deficiency is anxiolytic already in pups. V1b receptors are the most important players in connection with their ACTH-regulatory role, but a combination of V1aR-V1bR antagonist might be also beneficial through other mechanism, reducing the possibility of side effects. In contrast, antagonizing the V2 receptors may be stressful due to an induction of imbalance in salt-water homeostasis.

In conclusion, we confirmed the involvement of both V1bRs and V1aRs in the anxiolytic effect of vasopressin, without contribution of V2Rs. Stress axis changes might contribute to the observed anxiolysis, but only in part. Take into consideration the possible side effects, a mixed V1aR-V1bR antagonist treatment might be more beneficial than either antagonist alone.

\section{9}

\section{Can peer-to-peer psychoeducation be a useful support} for people with anxiety disorders?

Yvonne Kussinger ${ }^{1}$, Kerstin Schäffer ${ }^{2}$, PD Dr. rer. biol. hum. Dipl. Psych. Gabriele Pitschel-Walz ${ }^{1}$

${ }^{1}$ Technical University of Munich, School of Medicine, Klinikum rechts der Isar, Department of Psychiatry and Psychotherapy, Munich, Germany, ${ }^{2}$ Münchner Angstselbsthilfe MASH, Munich, Germany

Background: In Germany as well as worldwide, anxiety disorders have a high prevalence, cause great costs and represent a considerable impairment in quality of life for those affected. Although cognitive behavioural therapy is an effective method of treatment, the proportion of people who make use of it is low. One reason is the lack of therapy places and the resulting long waiting lists. The stigma associated with mental illness is another impediment. Both professionally led psychoeducation and self-help groups have been shown to support people with anxiety disorders. From the combination of these two 
techniques, a low-threshold offer for people with anxiety disorders can be created.

Method: In cooperation with the Münchner Angstselbsthilfe (MASH), a self-help association for anxiety disorders, we developed and implemented a peer-to-peer psychoeducation programme. People who are affected by anxiety disorders themselves were trained as group leaders for psychoeducational groups. In six sessions, they taught their peers psychoeducation on the topic of anxiety. In a prepost intervention study, we tested whether anxiety and depression scores had decreased after participation in this programme, as well as whether self-efficacy expectations had increased. For this purpose, we used the State Anxiety Scale (STAI-G X1) for anxiety scores, the Beck Depression Inventory (BDI-II) for depression and the Self-Efficacy Scale (SWE) for self-efficacy expectations. In addition, we conducted a qualitative evaluation of the programme to assess possible weaknesses and areas for improvement.

Results: A total of 22 people participated in the peer-to-peer psychoeducational groups; complete data sets were available for 18 people. Anxiety scores decreased in $39 \%$ of the participants $(\mathrm{t}(17)=0.53, \mathrm{p}=.602$, effect size $\mathrm{d}=0.13)$. Depression scores decreased for $78 \%$ of the participants $(\mathrm{t}(17)=-3.27, \mathrm{p}=.004$, effect size $d=0.77)$. In addition, self-efficacy expectations increased in $61 \%$ of the participants $(\mathrm{t}(17)=2.43, \mathrm{p}=.027$, effect size $\mathrm{d}=0.57)$. The formative evaluation showed that all participants experienced the groups as 'helpful' (67\%) or 'very helpful' (33\%). Furthermore, most participants stated that attending the group has increased their knowledge on the topic of anxiety 'very much' (16.7\%) or 'much' $(66.7 \%)$, while $16.7 \%$ of respondents stated that their knowledge has 'hardly increased'. The majority of participants also rated the length $(55.6 \%)$ and number $(83.3 \%)$ of the group meetings as appropriate. Conclusions: The peer-to-peer psychoeducation on anxiety was well received by the participants and brought statistically significant improvements in depression and self-efficacy expectations. The changes in anxiety scores were not statistically significant; a followup study with a larger sample and a follow-up after a longer period would be useful here. In the qualitative evaluation, the response of the group was predominantly positive. Overall, peer-to-peer psychoeducation provides a good low-threshold offer for people with anxiety disorders. Limiting factors in this study were the pre-post intervention design without a control group, and the small sample size.

\section{0}

\section{Detection of stress and anxiety with state-of-the-art sensor technology in the telemedical treatment of obsessive-compulsive disorder in children and adolescents (SSTeP-KiZ)}

Carolin Hohnecker ${ }^{1}$, Karsten Hollmann ${ }^{1}$, Prof. Dr. Annette Conzelmann $^{1}$, Dr. Jan Kühnhausen ${ }^{1}$, Anna Haigis ${ }^{1}$, Annika Alt ${ }^{1}$, Dr. Winfried $\mathrm{Ilg}^{2}$, Prof. Dr. Martin Giese ${ }^{2}$, Helene Dick ${ }^{3}$, Prof. Dr. Christian Ernst ${ }^{3}$, Prof. Dr. Enkelejda Kasneci ${ }^{4}$, Björn Severitt ${ }^{4}$, Dr. Dr. Martin Holderried ${ }^{5}$, Prof. Dr. Wolfgang Bethge ${ }^{6}$, Martin Schurer ${ }^{7}$, Sven Sender ${ }^{7}$, Dr. Heinrich Lautenbacher ${ }^{8}$, Ursula Wörz ${ }^{8}$, Jonas Primbs $^{9}$, Prof. Dr. Michael Menth ${ }^{9}$, Dr. Gottfried Barth ${ }^{1}$, Prof. Dr. Tobias Renner ${ }^{1}$

${ }^{1}$ Child and Adolescent Psychiatry, Tübingen, Germany, ${ }^{2}$ Department of Cognitive Neurology, Hertie Institute for Clinical Brain Research, Tübingen, Germany, ${ }^{3}$ Chair of Economics and Management of Social Services within the Institute of Health Care and Public Management, Hohenheim, Germany, ${ }^{4}$ Department of Computer Science, HumanComputer Interaction, Tübingen, Germany, ${ }^{5}$ Central Division Medicine: Structure, Process and Quality Management, Tübingen,
Germany, ${ }^{6}$ Center for Clinical Studies, Tübingen, Germany, ${ }^{7}$ Data Protection, Tübingen, Germany, ${ }^{8}$ Department of Information Technology, Tübingen, Germany, ${ }^{9}$ Department of Computer Science, Tübingen, Germany

Background: Telemedicine interventions enable the enhancement of state-of-the-art behavioral treatment for OCD by allowing therapy to be delivered in the patient's immediate environment. The goal of SSTeP-KiZ is to further develop online-based treatment by using advanced sensor technology during exposure exercises conducted at patients' homes, through which a more valid symptom actualization is made possible. Of critical importance here is the recording of relevant emotional states such as stress and anxiety during the therapy session, which are reliably quantified by combining different sensor modalities. This will allow the therapy process to be directly and individually adapted to the patient and the situation, thus optimizing the success of the treatment. In addition, access to experts is made possible even in rural areas and the inhibition threshold to seek therapy can be reduced. In a preliminary study, our research group has already been able to demonstrate the efficiency of using telemedical access.

Methods: It is planned to establish the therapy system on a sample of 10 healthy children and 6 children with obsessive-compulsive disorder aged 12-18 years and to subsequently evaluate it by treating 20 children with obsessive-compulsive disorder aged 12-18 years. Patients receive weekly therapy sessions over a 14 -week period via teleconferencing with the children and parents. During the sessions and exposures, patients' field of view is recorded via eye trackers, measures of stress responses via heart rate and pupillometry, and movement measures for approach-avoidance behaviors. Using an AI approach, these indicators are integrated and reported back to the therapist online to optimize the therapy process. Accompanying appbased daily symptoms will also be collected by the children and parents and processed for use in the therapy process.

Expected results: We expect a good feasibility and significant symptom reduction by this therapeutic approach and the chance to make this system usable for broad clinical application.

Discussion: This approach can overcome limitations of internet-based interventions, as state-of-the-art sensor technology can compensate for difficult visual conditions or patient problems in naming emotional experience. The inhibition to access psychotherapeutic help due to shame or difficult access to experts can also be lowered. An adaptation to other disorders is planned.

\section{1}

\section{Threat belief determines the degree of costly safety behavior: assessing rule-based generalization of safety behavior with a dimensional measure of avoidance}

\author{
Dr Alex Wong ${ }^{1}$, Prof. Dr. Andre Pittig ${ }^{2}$ \\ ${ }^{1}$ Julius-Maximilians-Universität Würzburg, Würzburg, Germany, \\ ${ }^{2}$ Friedrich-Alexander-Universität Erlangen-Nürnberg, Erlangen, \\ Germany
}

Excessive generalization of safety behaviour to innocuous stimuli that resembled a feared stimulus is oftentimes maladaptive. Safety behaviour is conventionally assessed dichotomously, requiring multiple presentations of each test stimulus and examines the proportion of safety behaviour executed across all trials. Thus the generalization gradient confounds with ongoing extinction learning during non-reinforced test trials. The present study employed a recently developed dimensional measure of avoidance to examine the generalization of safety behaviour, which can be seen as the extent of safety behavior 
engagement. After acquiring fear and safety behaviour to a fear-related conditioned stimulus (CS + ), participants were presented with novel generalization stimuli (GSs) that were perceptually similar to the $\mathrm{CS}+$ to different extents. Safety behavior, threat expectancy ratings, and skin conductance responses were measured. We found a range of distinct generalization gradients in safety behaviour and threat expectancy ratings, which were highly consistent with participants' reported relational rules. Importantly, a dimensional measure of avoidance was able to examine the generalization gradients of safety behaviour even when each test stimulus was presented once, thus minimizing the effect of ongoing extinction learning. In addition, the generalization rules parallel how clinically anxious individuals develop different threat beliefs after trauma exposure.

\section{2}

\section{The riskiest psychological intervention? Positive and negative side effects of exposure for anxiety disorders}

Ingmar Heinig $^{1}$, Prof. Dr. Susanne Knappe ${ }^{1}$, Prof. Dr. Hans-Ulrich Wittchen $^{2}$, Prof. Dr. Jürgen Hoyer ${ }^{1}$, Prof. Dr. Volker Arolt ${ }^{3}$, Prof. Dr. Jürgen Deckert ${ }^{4}$, Prof. Dr. Katharina Domschke ${ }^{5}$, Prof. Dr. Tilo Kircher $^{6}$, Prof. Dr. Jürgen Margraf ${ }^{7}$, Prof. Dr. Benjamin Straube ${ }^{6}$, Prof. Dr. Andreas Ströhle ${ }^{8}$, Prof. Dr. Alfons Hamm ${ }^{9}$, Prof. Dr. Andre Pittig $^{10}$

${ }^{1}$ TU Dresden, Dresden, Germany, ${ }^{2}$ Ludwig-Maximilians-Universität München, Department of Psychiatry and Psychotherapy, Munich, Germany, ${ }^{3}$ Institute for Translational Psychiatry, University of Münster, Münster, Germany, ${ }^{4}$ Department of Psychiatry, Psychosomatics and Psychotherapy, Center of Mental Health, University Hospital Würzburg, Würzburg, Germany, ${ }^{5}$ Department of Psychiatry and Psychotherapy, Medical Center-University of Freiburg, Facul-ty of Medicine, Freiburg, Germany, ${ }^{6}$ Department of Psychiatry and Psychotherapy \& Center for Mind, Brain and Behavior-CMBB, Philipps-University Marburg, Marburg, Germany, ${ }^{7}$ Mental Health Research and Treatment Center, RuhrUniversität Bochum, Bochum, Germany, ${ }^{8}$ Department of Psychiatry and Psychotherapy, Campus Charité Mitte, CharitéUniversitätsmedizin Berlin, Berlin, Germany, ${ }^{9}$ Department of Psychology, Biological and Clinical Psychology, University of Greifswald, Greifswald, Germany, ${ }^{10}$ Translational Psychotherapy, Department of Psychology, Friedrich-Alexander University Erlangen-Nürnberg (FAU), Erlangen, Germany

Despite striking empirical support, exposure-based treatments for anxiety disorders are underused. This is in part driven by clinicians' concerns that patients may experience severe side effects, particularly in intensive forms of exposure. Yet, empirical data on side effects of exposure are scarce.

We therefore examined positive and negative side effects of exposure in a randomized controlled trial comparing standard vs. temporally intensified exposure ( 1 vs. 3 sessions/week). We analyzed data from 607 patients with panic disorder, agoraphobia, social anxiety disorder and multiple specific phobias who completed on average 7.9 exposure exercises during the trial. Exercises were designed to maximize prediction error in order to increase inhibitory learning. Side effects included treatment-emergent effects as well as therapeutic malpractice and were measured with the Inventory for the Balanced Assessment of Negative Effects of Psychotherapy (INEP).

$94.1 \%$ of patients reported positive side effects; $42.2 \%$ reported negative effects, most frequently treatment stigma (16.6\%), negative feelings $(14.8 \%)$ and the experience to depend on the therapist $(10.9 \%) .3 .3 \%$ of patients reported therapeutic malpractice.
Intensified exposure was not associated with higher negative effects. Negative effects were mostly of minor intensity and were frequently unrelated to exposure (e.g., treatment stigma, consequences for insurance policies). Negative effects were not associated with demographic variables or subsequent dropout, but with pre-treatment and anxiety severity. Additionally, the presence of negative effects predicted lower symptom reduction at post and 6-month follow-up. In sum, results encourage the use of exposure in general, even in temporally condensed variants.

The present content can alternatively be presented as a poster or e-poster.

\section{3}

\section{Diurnal dynamics of stress and mood in the risk groups during COVID-19 lockdown: a large binational ecological momentary assessment study}

\section{Bsc Msc Ekaterina Pronizius ${ }^{* 2}$, BSc MSc Anja Christine} Feneberg*1,3 , B.A. MSc PhD Paul A. G. Forbes ${ }^{2}$, Univ.-Prof. Mag. Dr. Claus Lamm*2,3 , Univ.-Prof. Dr. Urs Markus Nater*1,3, BSc MSc Giulio Piperno*1,4, Assoz. Prof., Privatdoz. PhD Giorgia Silani*1,3, Dipl.-Psych. Dr. Nadine Skoluda ${ }^{1,3}$, BSc MSc Ana Stijovic*1

${ }^{1}$ University of Vienna, Faculty of Psychology, Department of Clinical and Health Psychology, Vienna, Austria, ${ }^{2}$ University of Vienna, Faculty of Psychology, Department of Cognition, Emotion, and Methods in Psychology, Vienna, Austria, ${ }^{3}$ University of Vienna, Faculty of Psychology, University of Vienna Research Platform „The Stress of Life-Processes and Mechanisms underlying Everyday Life Stress “ (SOLE), Vienna, Austria, ${ }^{4}$ Sapienza University of Rome, Faculty of Medicine and Psychology, Department of Psychology, Rome, Italy

The global pandemic of COVID-19 is accompanied by nationwide constraints, which disrupt the daily routine to a great extent. As many countries are struggling to prevent the spread of the virus, further lockdowns may still occur. It is therefore of both theoretical and practical importance to identify factors that make people vulnerable to the negative psychological effects of lockdown. The present study is part of an ongoing larger preregistered project (osf.io/gsvdf), which contains multiple bursts. In the first study burst, we measured momentary fluctuations in stress and mood during the acute phase of the lockdown in Austria and Italy in April 2020 using an ecological momentary assessment (EMA) approach. 731 participants (70.5\% females, Mage $=31.7$, SDage $=11.7$, Austria $n=480$, Italy $n=225$ ) entered their stress, mood levels, and recent behaviours into a smartphone app (movisensXS) five times per day for seven days. In addition to subjective data, we assessed hair cortisol concentration $(\mathrm{HCC})$ in a sample subset from Austria $(\mathrm{n}=136,75.47$ females, Mage $=34.45$, SDage $=12.64)$. We used $\mathrm{HCC}$ as a valid and reliable biological marker for chronic stress.

We showed that higher trait loneliness, higher scores in chronic stress and depressive symptoms were associated with momentary increases in stress and/or lower mood (valence, calmness, or energy) across the study period. As the day progressed, the participants generally showed a decline in stress. However, this reduction in stress was attenuated in younger participants and less financially secure individuals. We observed a flattened diurnal curve for energy in participants with more depressive symptoms and higher trait loneliness, who were hypo-aroused earlier in the day and hyper-aroused in the evenings. Having similar diurnal dynamics for energy, they showed an opposite pattern in mood valence. As the day progressed, the participants with more depressive symptoms reported a slight increase in mood valence, whereas more lonely participants showed a 
decline in mood valence and tended to be less calm. A comparison of the HCC levels from before to during the lockdown revealed a decrease in HCC levels. None of the risk factors was significantly associated with the change in HCC from pre- to during the pandemic.

Our study is in line with previous work showing that the psychological wellbeing of young and isolated individuals and those suffering from psychiatric conditions could be most affected by COVID-19 restrictions. The study highlights the need for tailored support to these vulnerable groups when the restrictions are in place but also for the period after that. A decrease of HCC levels from before to during the pandemic, found in our study contradicts previous empirical findings, which reported an increase of the HCC levels during the same period. A possible confound of the effects with seasonal changes and/or reduced physical activity in our sample might explain this conflicting finding. Our results emphasize the need for future studies to include additional physiological measures of stress and to address its relationship with psychological measures in different risk groups during times of uncertainty.

\section{4 \\ Age related exacerbation of innate fear in the 3xTg-AD mouse model of Alzheimer's disorder}

Adrienn Szabó ${ }^{1,2}$, Bibiána Török ${ }^{1,2}$, Csilla Lea Fazekas ${ }^{1,2}$, Krisztina Bánrévi $^{1}$, Pedro Correia ${ }^{1,2}$, Tiago Chaves ${ }^{1,2}$, M.D. Ph.D. DSc. Dóra Zelena $^{1,3}$

${ }^{1}$ Institute of Experimental Medicine, Budapest, Hungary, ${ }^{2} J a ́ n o s$ Szentágothai Doctoral School of Neurosciences, Semmelweis University, Budapest, Hungary, ${ }^{3}$ Centre for Neuroscience, Szentágothai Research Centre, Institute of Physiology, Medical School, University of Pécs, Pécs, Hungary

Alzheimer's disorder (AD) is a serious health and societal problem with unsolved treatment. It is often comorbid with other psychiatric illnesses, like anxiety. In order to develop new treatment options, it is necessary to find a proper animal model of these comorbidities. Transgenic mice bearing human mutation known to induce AD are the most widely used models for this disorder.

Our present aim was to follow the appearance of innate fear in the triple-transgenic mouse model of $\mathrm{AD}(3 \mathrm{x}-\mathrm{Tg} \mathrm{AD})$ comparing 2-4-6-812-15-18-month-old animals with age-matched controls. First, we used open field test to check locomotor activity, as well as to reveal if they spend less time in the central part of the test box as a sign of anxiety. Next, we assessed the innate fear with fox odour test.

Locomotor impairment was already present in 2-month-old animals (both males and females) and remained detectable during the entire spectrum of the studied 18 month. Interestingly, the animals spent more (and not the expected less) time in the central part of the open arena. However, this is likely due to the reduced locomotor activity, which was reflected by the lower frequency of centrum entries. Fox odour induced freezing and avoidance behaviour in all animals. However, with aging the AD animals were more distressed than their controls, suggesting a gradual exacerbation of innate fear. There were no significant differences between the groups in the resting adrenocorticotropic hormone and corticosterone levels measured during the beginning of the active phase, thus, stress-hormone changes is unlikely to contribute to this phenomenon.

In summary, despite motor impairment in open field test, the freezing and avoidance behaviour during fox odour test seem to be a reliable parameter to examine the comorbidity of anxiety in $3 \mathrm{x}-\mathrm{Tg}$ $\mathrm{AD}$ mice. The symptoms develop gradually, which might resemble the human situation.
45

\section{Daily life stress and the cortisol awakening response over a 14-months stress phase: findings from the LawSTRESS project}

Hannah L. Peter ${ }^{1}$, Marina Giglberger ${ }^{1}$, Sandra Zänkert ${ }^{1}$, Dr. GinaIsabelle Henze ${ }^{1}$, Christoph Bärt ${ }^{1}$, Julian Konzok ${ }^{1}$, Prof. Dr. Brigitte M. Kudielka ${ }^{1}$, Prof. (apl.) Dr. Stefan Wüst ${ }^{1}$

${ }^{1}$ University of Regensburg, Regensburg, Germany

The objective of the prospective-longitudinal and quasi-experimental LawSTRESS project is to contribute to the understanding of the biopsychological mechanisms mediating the well-known association between chronic stress and the risk for several disorders. In the present analysis, we focused on the association between daily life stress and the cortisol awakening response (CAR) over a long-lasting stress phase. The CAR is a well-established marker of cortisol regulation in psychoneuroendocrinology.

In this project, law students from Bavarian universities (total $\mathrm{n}=471$ ) have been studied over a 14-months period. The stress group (SG) consists of students experiencing a long-lasting and significant stress phase, namely the preparation for the final state examination for law students. Participants assigned to the comparison group (CG) were studied over an equally long period without particular stress exposure. The study protocol included six sampling points with the first assessment (T1) taking place twelve months, the second (T2) three months and the third (T3) one week prior to the exam. Time point four (T4) took place during the exam and time points five and six (T5, T6) one week and one month after the exam.

To investigate stress-related alterations in the CAR, we examined a subsample of the LawSTRESS project consisting out of 204 students, 97 subjects from the SG $(69.1 \%$ female, mean age $=22.84 \pm 1.82)$ and 107 from the CG $(78.5 \%$ female, mean age $=20.95 \pm 1.93)$. At each sampling point, the CAR was assessed via saliva samplings directly after awakening and 30 as well as $45 \mathrm{~min}$ later. Stress perception in daily life was measured via repeated ambulatory assessments of the AA stress index, a newly developed scale consisting of 5 items.

Subjective stress and the CAR were predicted with two-level linear mixed models. The time course of the stress perception in the two groups differed significantly, with the SG showing an increase in the AA stress index until the exam and a decrease thereafter. Regarding the CAR, cortisol levels after awakening showed the expected increase with no significant difference between groups at the first sampling point. Remarkably, we found significant alterations in the linear time trend of the CAR in SG and CG over the 14 monthperiod. In the SG, the increase of cortisol after awakening was flattened at $\mathrm{T} 2, \mathrm{~T} 3$ and $\mathrm{T} 4$ compared to $\mathrm{T} 1$. This alteration in the CAR was not associated with the increase of the AA stress index in the SG.

Our results document that the preparation for the final state examination was related to a significant increase of perceived stress. Furthermore, the findings support the view that chronic stress is related to a blunted CAR. We did not find a significant relation between stress response domains, i.e., between the CAR as index of hypothalamic-pituitary-adrenal axis regulation and the AA stress index measuring perceived stress in everyday life. 
46

\section{Daily life stress and the cortisol awakening response over a 14-months stress phase: findings from the LawSTRESS project}

Hannah Peter ${ }^{1}$, Marina Giglberger ${ }^{1}$, Sandra Zänkert ${ }^{1}$, Gina-Isabelle Henze $^{1}$, Christoph Bärtl ${ }^{1}$, Julian Konzok ${ }^{1}$, Prof. Dr. Brigitte M. Kudielka $^{1}$, Prof. (apl.) Dr. Stefan Wüst ${ }^{1}$

${ }^{1}$ University of Regensburg, Regensburg, Germany

The objective of the prospective-longitudinal and quasi-experimental LawSTRESS project is to contribute to the understanding of the biopsychological mechanisms mediating the well-known association between chronic stress and the risk for several disorders. In the present analysis, we focused on the association between daily life stress and the cortisol awakening response (CAR) over a long-lasting stress phase. The CAR is a well-established marker of cortisol regulation in psychoneuroendocrinology.

In this project, law students from Bavarian universities (total $\mathrm{n}=471$ ) have been studied over a 14-months period. The stress group (SG) consists of students experiencing a long-lasting and significant stress phase, namely the preparation for the final state examination for law students. Participants assigned to the comparison group (CG) were studied over an equally long period without particular stress exposure. The study protocol included six sampling points with the first assessment (T1) taking place twelve months, the second (T2) 3 months and the third (T3) 1 week prior to the exam. Time point four (T4) took place during the exam and time points five and six (T5, T6) 1 week and 1 month after the exam.

To investigate stress-related alterations in the CAR, we examined a subsample of the LawSTRESS project consisting out of 204 students, 97 subjects from the SG $(69.1 \%$ female, mean age $=22.84 \pm 1.82)$ and 107 from the CG $(78.5 \%$ female, mean age $=20.95 \pm 1.93$ ). At each sampling point, the CAR was assessed via saliva samplings directly after awakening and 30 as well as $45 \mathrm{~min}$ later. Stress perception in daily life was measured via repeated ambulatory assessments of the AA stress index, a newly developed scale consisting of 5 items.

Subjective stress and the CAR were predicted with two-level linear mixed models. The time course of the stress perception in the two groups differed significantly, with the SG showing an increase in the AA stress index until the exam and a decrease thereafter. Regarding the CAR, cortisol levels after awakening showed the expected increase with no significant difference between groups at the first sampling point. Remarkably, we found significant alterations in the linear time trend of the CAR in SG and CG over the 14 monthperiod. In the SG, the increase of cortisol after awakening was flattened at T2, T3 and T4 compared to T1. This alteration in the CAR was not associated with the increase of the AA stress index in the SG.

Our results document that the preparation for the final state examination was related to a significant increase of perceived stress. Furthermore, the findings support the view that chronic stress is related to a blunted CAR. We did not find a significant relation between stress response domains, i.e., between the CAR as index of hypothalamic-pituitary-adrenal axis regulation and the AA stress index measuring perceived stress in everyday life.
47

\section{Coping and social support as predictors of efficient control of psychosocial stress in underage unaccompanied refugees in Würzburg, Germany}

\author{
Dr. Phil. Hina Ghafoor ${ }^{1,4}$, Mr Markus Schulz ${ }^{1}$, Prof. Dr. Paul \\ Pauli $^{1,3}$, Prof. Dr. Stefan Schulz ${ }^{1,2}$ \\ ${ }^{I}$ Department of Psychology I, Biological Psychology, Clinical \\ Psychology and Psychotherapy, Würzburg, Germany, \\ ${ }^{2}$ Comprehensive Heart Failure Center, Würzburg, University \\ Hospital of Würzburg, Würzburg, Germany, ${ }^{3}$ Center of Mental \\ Health, University of Würzburg, Würzburg, Germany, ${ }^{4}$ Riphah \\ International University, Islamabad, Pakistan
}

Coping with psychosocial stress caused by forced migration among underage unaccompanied refugees may benefit from family support and emotional intelligence. Germany has recently witnessed a surge of underage unaccompanied refugees $(35,939)$ between the year 2015 and 2016.

The current study has examined the roles of social support and coping mechanisms on the performance efficiency of underage unaccompanied refugees. For this purpose, a sample of $\mathrm{N}=32$ underage unaccompanied refugees (age; $\mathrm{M}=18.84$ years) was recruited from German Youth Welfare Centers in Würzburg, Ochsenfurt, and Aschaffenburg for a cross-sectional survey. Mixed method analysis was applied to evaluate both quantitative and qualitative responses.

The results indicated that more positive coping $(\mathrm{p}=0.03)$ and higher social support $(\mathrm{p}=0.04)$ predicted greater creativity and productivity in controlling distressing aspects of their experiences (house-tree-person test: efficiency quotient). Social support provided by mentors in the Youth Welfare Centers enhanced processes of acculturation (integration and assimilation).

It can be concluded from the current study that supporting positive coping and providing social support (regardless of whether it is provided by family or institutional professionals (mentors)) helps underage unaccompanied refugees to better utilize their maximum potentials in support of adjusting to a new culture.

Keywords: Underage unaccompanied refugees, Psychosocial Stress, Social support, Positive coping, Performance efficiency

\section{8}

\section{Anxiety and cardiovascular responses in everyday social interactions}

Marthe Gründahl $^{1}$, Dr. Martin Weiß ${ }^{1}$, Prof. Dr. Jürgen Deckert ${ }^{1}$, Prof. Dr. Grit Hein ${ }^{1}$

${ }^{1}$ University Clinic Würzburg, Department of Psychiatry, Psychosomatics and Psychotherapy, Translational Social Neuroscience Unit, Würzburg, Germany

Interactions with others affect our physical and mental well-being and functioning. Despite their relevance in psychological research, it is a challenge to authentically reconstruct social interactions in the laboratory. Ecological Momentary Assessment (EMA) allows us to investigate the effects of social interactions in the most realistic setting: everyday life.

In an on-going study, we investigate the effects of different interaction partners and settings on state anxiety and related changes in heart rate (HR) and heart rate variability (HRV) via smartphones and portable electrocardiogram (ECG) sensors. On five consecutive 
days, participants (aged 18-35) answer six randomly timed surveys a day while continuously wearing an ECG chest belt.

Based on our previous findings in the laboratory, we expected a positive correlation of anxiety (state and trait) with HR, and a negative correlation with HRV. Moreover, we hypothesized that less anxious individuals would benefit from more similar and more familiar interaction partners as indicated by decreased HR and increased HRV. The opposite was expected for anxious individuals. These effects should be stronger in direct, face-to-face contacts compared to virtual interactions. However, for individuals with high social interaction anxiety, virtual contacts should lead to stronger stress-reducing effects.

Preliminary results $(\mathrm{N}=32$ by May 2021) suggest that social interaction anxiety is associated with higher HR during social interactions. We found no effect of anxiety on HRV. In contrast, similarity and familiarity have a substantial stress-reducing effect on both HR and HRV. High social interaction anxiety is related to stronger physiological effects of virtual contacts. However, contrary to our expectations, highly anxious individuals benefit more from highly similar interaction partners. We look forward to exploring the stability of these effects in a bigger sample.

\section{9}

\section{The role of social processing abilities in acute psychosocial stress.}

\section{Jost Blasberg $^{1}$ \\ ${ }^{1}$ University Clinic Jena, Jena, Germany}

The role of social processing abilities in acute psychosocial stress Jost U. Blasberg

Institute of Psychosocial Medicine, Psychotherapy and Psychooncology, Jena University Hospital, Friedrich-Schiller University, Jena, Germany

Symposium of Neuroscience of stress

Chair: J. Prüssner, Konstanz and V. Engert, Jena

By activating the sympathetic nervous system and hypothalamic-pituitary-adrenal axis over a long period of time, chronic psychosocial stress can have detrimental effects on mental and bodily health. If social interactions are perceived as ego-threatening, an assessment driven by individual social capacities, psychosocial stress ensues. In the current study, we investigated whether basic aspects of social processing are associated with the stress response to an acute psychosocial stressor. Empathy, compassion, and Theory of Mind (ToM) ability were appraised using a state-of-the-art paradigm, the EmpaToM. Self-reported other-and self-compassion were measured by means of conventional paper-pencil questionnaires. Subjects $(\mathrm{N}=119)$ also experienced the Trier Social Stress Test (TSST), a standardized psychosocial laboratory stressor. Stress reactivity and recovery were assessed in terms of salivary cortisol and alpha-amylase, heart-rate, high-frequency heart-rate variability (HF-HRV), and subjective experience. Hierarchical regression models were calculated in a Bayesian framework. The analysis showed that different aspects of social processing were linked to different aspects of psychosocial stress experience, with stress-buffering effects of ToM on HF-HRV reactivity, and EmpaToM-derived compassion on subjective stress recovery. Concerning questionnaire data, self-compassion was related to reduced subjective stress reactivity, and, in individuals with low levels of other-compassion, concordantly low EmpaToM-derived empathy ratings went along with increased cortisol reactivity, compared to subjects with higher empathy. We conclude that greater social processing capabilities reduce the experience of psychosocial stress. Actively increasing the tendency to understand and react to others' affections not only benefits the ones closest to us, but may protect ourselves from ego-threatening social situations, and, longterm, from the development of stress-related disease.

\section{0}

\section{The effect of reappraisal on subjective and neuroendocrine stress responses in women with varying exposure to early-life maternal care}

Ulrike U. Bentele ${ }^{1}$, Maria Meier ${ }^{1}$, Annika B. E. Benz ${ }^{1}$, Bernadette Denk $^{1,3}$, Stephanie Dimitroff ${ }^{1}$, Eva Unternaehrer ${ }^{1,2}$, Jens C. Pruessner ${ }^{1,3}$

${ }^{1}$ Department of Psychology, University of Konstanz, Konstanz, Germany, ${ }^{2}$ Child- and Adolescent Research Department, Psychiatric University Hospitals Basel (UPK), University of Basel, Basel, Switzerland, ${ }^{3}$ Centre for the Advanced Study of Collective Behaviour, University of Konstanz, Konstanz, Germany

Introduction: Appraisal processes play an important role in the regulation of subjective and endocrine responses to stressful or emotional situations. Cognitive reappraisal has been proven an efficient strategy in attenuating subjective emotional responses to stress. However, results regarding the effect on endocrine stress responses are more heterogenous and point to additional moderating factors. One such factor that has repeatedly been associated with altered hypothalamic-pituitary-adrenal (HPA) axis responses is exposure to adverse early-life experiences, such as low extent of maternal care (MC). Thus, the aim of the current study was to investigate the effects of instructed reappraisal and $\mathrm{MC}$ on subjective and cortisol responses to psychosocial stress.

Methods: Sixty healthy young women $($ mean $($ age $)=20.53$, sd $($ age $)=2.49$ ) participated in the study. Prior to the experiment, participants were assigned to either a low $(n=18)$ or high $(n=42)$ MC group, based on the self-reported extent of MC during childhood and adolescence using the Parental Bonding Instrument. Upon arrival, participants either received written instructions to use cognitive reappraisal (reappraisal group, $\mathrm{n}=29$ ) or to focus on their senses (control group, $\mathrm{n}=31$ ) during the subsequent stress task. Thereafter, participants underwent the Trier Social Stress Test. Salivary cortisol and subjective stress levels were measured repeatedly throughout the experimental session. Analyses were conducted using multilevel mixed models.

Results: We found that subjective stress ratings increased prior to the TSST and decreased during the recovery period. However, results did not show a main effect of or an interaction between reappraisal and MC. Pending results on cortisol responses will be reported and discussed at the time of poster presentation.

Discussion: These preliminary results do not confirm any beneficial effect of reappraisal on subjective stress responses. However, final results will allow for a more global interpretation and better understanding of the complex association between reappraisal and HPA axis responses. Moreover, they might provide insight into whether specific cognitive interventions might prove beneficial for people with a history of early-life adversity. 
51

\section{Effects of the latest version of the FRIENDS for LIFE prevention program in children at high risk for the development of an anxiety disorder}

\author{
Katharina Kneer ${ }^{1}$, Prof. Dr. Marcel Romanos ${ }^{1}$, Prof. Dr. Katharina \\ Domschke $^{2}$, PD Dr. Susanne Neufang ${ }^{3,4}$ \\ ${ }^{1}$ German Center of Prevention Research in Mental Health; Julius- \\ Maximilians-University Würzburg; c/o Center of Mental Health; \\ Department of Child and Adolescent Psychiatry, Psychosomatics \\ and Psychotherapy; University Hospital of Würzburg, Würzburg, \\ Germany, ${ }^{2}$ Department of Psychiatry and Psychotherapy; Medical \\ Center-University of Freiburg; Faculty of Medicine; University \\ of Freiburg, Freiburg, Germany, ${ }^{3}$ Department of Psychiatry \\ and Psychotherapy; Medical Faculty; Heinrich-Heine University, \\ Düsseldorf, Germany, ${ }^{4}$ Comparative Psychology; Institute \\ of Experimental Psychology; Heinrich-Heine University, Düsseldorf, \\ Germany
}

Background: Depression and Anxiety disorders are the most prevalent groups of psychiatric disorders (Stein 2017; Kessler 2012) and go along with personal suffering and high socioeconomic burden (Guo 2019; Kessler 2012). Since half of all mental disorders show their onset before 14 years of age (Kessler et al. 2007) development and evaluation of effective preventative approaches targeting children at risk are urgently warranted to reduce the incidence of both disorder groups.

In this study, we evaluated the effect of a shortened version of the FRIENDS for LIFE prevention program on anxiety measures as well as on depressive symptoms in children at high risk to develop anxiety disorders in an indicated prevention approach.

Methods: 21 typically developing, but highly anxiety sensitive children (aged from 8 to 12) underwent a shortened version of the most recent adaptation of the FRIENDS for LIFE prevention program. To quantify the effect of the prevention program, anxiety sensitivity (Childhood anxiety sensitivity index, [CASI]), trait anxiety (StateTrait-Anxiety-Inventory for children, Trait version, [STAIC]), separation anxiety (Trennungs-Angst Inventar, [TAI]) as well as depressivity (Depressionsinventar für Kinder und Jugendliche, $[\mathrm{DIKJ}])$ were used. Anxiety and depression assessment were performed before (T0), directly after (T1) and 6-month following (T2) the intervention. The prevention success for all measures was analyzed using repeated measures analysis of variance.

Results: Preliminary results of the effects of the prevention revealed a reduction of all anxiety parameters and depressivity, which was statistically significant for anxiety parameters (CASI: $(\mathrm{F}(2,40)=3.7$, $\mathrm{p}=.03$; STAIC: $(\mathrm{F}(2,40)=3.6, \mathrm{p}=.03$; TAI $(\mathrm{F}(2,40)=\mathrm{T}=6.3$, $\mathrm{p}=.00)$, but not depressivity (DIKJ: $(\mathrm{F}(2,40)=0.6, \mathrm{p}=.55)$. Posthoc pairwise comparisons revealed that for STAIC and CASI the reduction was significant from pre (T0) to post (T1) intervention assessment and for TAI from pre (T0) to 6 month follow-up (T2) assessment.

Conclusions: We conclude that in our small sample the shortened version of the FRIENDS for LIFE prevention program is effective in reducing anxiety symptoms in highly anxious children. While the effect was not statistically significant for depressive symptoms, short term improvements in anxiety sensitivity and trait anxiety, as well as long term effect for separation anxiety could be found.

\section{2}

Online-based acceptance and commitment therapy, and mindfulness-based cognitive therapy improves depression and anxiety in healthcare workers during COVID-19 outbreak

PsyD Amir Jahanian Najafabadi ${ }^{1}$, PsyD Shabnam Borhanizad ${ }^{2}$, PsyD Hadis Imani ${ }^{1}$, PysD Babak Vojoudi ${ }^{3}$, PsyD Nastaran Otared ${ }^{4}$

${ }^{1}$ Department of Psychology and Methods, Jacobs University Bremen, Bremen, Germany, ${ }^{2}$ Department of Psychology, University of Porto, Porto, Portugal, ${ }^{3}$ Department of Psychology, Tabriz University, Tabriz, Iran, ${ }^{4}$ Department of Psychology, University of Mohaghegh Ardebili, Ardebil, Iran

The aim of the current study was to examine the effect of acceptance and commitment therapy (ACT) and Mindfulness-Based Cognitive Therapy (MBCT) on anxiety and depressive symptoms of health care workers (HCWs) during the pressure of the COVID-19 Outbreak and whether their quality of life improves after group therapy. ACT has been developed as a behavioral therapy that cultivates mindfulness, acceptance, cognitive defusion, and consists of strategies to increase psychological flexibility and promote behavior changes consistent with personal values. MBCT as a cognitive behavioral therapy with mindfulness meditation practices helps a person to observe and recognise negative reactions and their surroundings in the present moment. This approach is to develop a greater sense of self-awareness and understanding of negative thoughts and it has been used on a wide range of psychological disorders. To examine our hypothesis, we recruited $40 \mathrm{HCWs}$ who had struggled with anxiety and depression and approached psychotherapy clinics located in Iran (Tabriz and Urmia city) during COVID-19 outbreak. Participants were divided randomly into two groups. One group received an online group-based ACT program, and one had received an online-based MBCT program. Participants were assessed by the Beck Depression Inventory (BDI, Second Edition), Beck Anxiety Inventory (BAI), Quality of Life Index (QOLI), Global Assessment of Functioning Scale (GAF), and The Acceptance and Action Questionnaire-II (AAQ-II).

Results of ANCOVA revealed significant effects of MBCT and ACT treatment on anxiety $(\mathrm{F}=14.03, \mathrm{p}<0.01, \mathrm{pEta} 2=0.27)$, depression $(\mathrm{F}=4.23, \mathrm{p}<0.05, \mathrm{pEta} 2=0.10)$ and acceptance and action $(\mathrm{F}=8.61, \mathrm{p}<0.05, \mathrm{pEta} 2=0.19)$, but no significant changes were obtained in quality of life scores. Results further revealed a significant difference between two groups in the variable of acceptance and action $(\mathrm{F}=7.65, \mathrm{p}<0.05)$ by comparing the modified means of the two methods. Moreover, ACT treatment showed greater effectiveness than MBCT treatment in the acceptance and action component. Here we conclude that ACT and MBCT support HCWs to improve their anxiety and depression symptoms during the COVID19 Outbreak and based on participant feedback, these approaches can be used in different settings for reduction of occupational stress and eliminate related negative thoughts in the workplace.

Keyword: Acceptance and Commitment Therapy, MindfulnessBased Cognitive Therapy, Anxiety, Depression, Quality of Life, Healthcare Workers, Covid-19. 
53

\section{The impact of play therapy to reduce anxiety related symptoms in school-age children during COVID-19 pandemic}

\author{
Sohrab Abdi Zarrin ${ }^{2}$, PsyD Amir Jahanian Najafabadi ${ }^{1}$ \\ ${ }^{1}$ Jacobs University Bremen, Bremen, Germany, ${ }^{2}$ Department \\ of Educational Sciences, University of Qom, Qom, Iran
}

COVID-19 pandemic is more than just a medical emergency; it has an impact on individuals and society, generating disruption, concern, stigma and fear. As recent studies revealed a strong impact on children and families especially when comes to an online teaching and stressful situations. Therefore, a non-pharmaceutical treatment is necessary to use for children at home. The aim of this experimental study was to investigate whether play therapy improve anxiety of school-age children during COVID-19 pandemic. Sixteen children were recruited from waiting list rereferred to psychological and counseling centers by psychiatrists in Iran. Participants were randomly assigned to the experimental (play therapy) and control group (no intervention) each consisted 8 children. Spence Children Anxiety Scale (SCAS) was used as a pre-test and post-test to assess the level of anxiety before and after the intervention. Each therapeutic session lasted $45 \mathrm{~min}$. Results showed the mean scores of pre-test and posttest were significantly different in the two groups, and play therapy were effectively reduced the level of anxiety in different dimensions (separation anxiety, social phobia, obsessive-compulsive disorder, panic disorder/agoraphobia, personal injury fears, generalized anxiety) in children $(p<0.01)$. Further analyses revealed a significant difference between the mean scores of anxiety disorders in experimental group compared with control group $(\mathrm{p}<0.01)$. Here we suggest play therapy as a promising scientific approach and low-cost treatment to be used to reduce anxiety symptoms in children.

Keywords: play therapy, anxiety, children, Covid-19,

\section{4}

\section{Anxiety related disorders are correlated with substance use: a clinical study}

MD Shiva Soraya ${ }^{1}$, MD Mahdieh Saeidi ${ }^{1}$, MD Seddigh Ruohollah ${ }^{2}$, MD Mehri Mahdavi ${ }^{1}$, MD Sara Nooraeen ${ }^{3}$, PsyD Amir Jahanian Najafabadi $^{3}$

${ }^{1}$ Research Center for Addiction and Risky Behaviors, Iran University of Medical Sciences, Tehran, Iran, Tehran, Iran, ${ }^{2}$ Spiritual Health Research Center, Iran University of Medical Sciences, Tehran, Iran, Tehran, Iran, ${ }^{3}$ Mental Health Research Center, School of Behavioral Sciences and Mental Health, Iran University of Medical Sciences, Tehran, Iran, ${ }^{4}$ Department of Psychology \& Methods, Jacobs University Bremen, Bremen, Germany (Corresponding author a.jahaniannajafabadi@jacobs-university.de),Bremen, Germany

In this study we investigated the prevalence of anxiety related disorders and its correlation with different substances used by patients diagnosed with substance use disorder referred to the Iran Psychiatric Hospital located in Tehran. We recruited 292 male patients aged 18-65 (Mean age $=36.11$, SD $=10.55$ ). Structured Clinical Interview for DSM Disorders (SCID) was used to investigate their anxiety related disorders and then correlated with the different substances used during a year before our study. Based on clinical evaluation and structured psychiatric interviews, we investigated panic disorder (PD), agoraphobia, society anxiety disorder (SAD), and general anxiety disorder (GAD). We also further investigated Diabetes
Mellitus, Thyroid Disease, Coronary Artery Disease, Convulsion, human immunodeficiency virus (HIV) and Hepatitis B virus (HBV), Hepatitis C, Other Medical Disease. Results revealed that SAD is significantly correlated with opioids $(p=0.005)$, methadone $(p=0.007)$, tramadol $(p<0.001)$ benzodiazepines $(p<0.01)$. Correlation analyses revealed PD was significantly correlated with LSD ( $\mathrm{p}<0.001)$, cannabis $(\mathrm{p}=0.047)$, and marginally correlated with alcohol and heroin $(\mathrm{p}<0.06)$. Agoraphobia was significantly correlated with IV drugs $(p=0.004)$. Further statistical analyses also revealed that GAD was significantly correlated with tramadol, daily smoking cigarettes and other street drugs $(p<0.01)$. Additional results and prevalence will be presented according to the secondary data analysis and will be discussed accordingly.

\section{5 \\ Hypothalamic-pituitary-adrenal axis reactivity to traumatic stress to predict the development of posttraumatic stress disorder symptoms: a systematic review of experimental studies}

Sinha Engel ${ }^{1}$, Sebastian Laufer ${ }^{1}$, Hannah Klusmann ${ }^{1}$, Lars Schulze ${ }^{1}$, Sarah Schumacher ${ }^{2}$, Christine Knaevelsrud ${ }^{1}$

\section{${ }^{1}$ Freie Universität Berlin, Berlin, Germany, ${ }^{2}$ Health and Medical University Potsdam, Potsdam, Germany}

Background: Most individuals who experience a traumatic event do not develop clinically significant posttraumatic stress disorder (PTSD) symptoms, but those who do suffer from intense strain and impairment. Identifying risk factors for PTSD development can help to provide individuals at risk for adverse outcome after trauma with targeted, early interventions and prevent symptom chronification. A growing body of research is using biomarkers to predict PTSD risk, with the hypothalamic-pituitary-adrenal (HPA) axis being the most frequently studied system. Cortisol concentrations, as measured before, as well as shortly after trauma have already been investigated as such prognostic biomarkers. Cortisol reactivity to a trauma cannot be assessed in real-life settings, but experimental paradigms can provide us with access to the biological underpinnings of trauma processing. Therefore, cortisol reactivity to experimentally-induced traumatic stress can be used as a prognostic marker for PTSD symptom development in laboratory settings. This is the first systematic review summarizing all empirical, experimental studies that related cortisol concentrations before, during or after exposure to stimuli with traumatic content to PTSD symptom development.

Methods: We included published and unpublished reports of studies which investigated humans without prior trauma exposure, exposed them to visual or auditory, traumatic stimuli (e.g. words, texts, pictures or movies), measured cortisol concentrations before, during or after stimulus presentation and subsequently measured PTSD symptoms. Reports in German or English language, of any year, were included. Exclusion criteria were: reports written in any other language; animal studies; studies explicitly investigating individuals with prior trauma exposure; studies using olfactory, haptic or nontraumatic stimuli; studies without cortisol assessment before PTSD symptom assessment; non-experimental studies and systematic reviews. To identify all eligible studies, we searched PubMed, PubPsych, PsychINFO, PsycArticle, Web of Science and EMBASE up to December, 21st 2020. We additionally searched for dissertations (ProQuest, February 15th 2021), unpublished studies (Clinicaltrials.gov, February, 16th 2021), examined the grey literature and applied snowball search methods. Data for the qualitative and quantitative analysis are currently being extracted, by means of standardized procedures. Eligible studies will be systematically described, 
including a quality assessment by a cortisol assessment checklist. For meta-analysis, correlations between indicators of cortisol reactivity to the traumatic stimuli and PTSD symptoms will be calculated. All methods are in accordance with the current PRISMA guidelines (Page et al. 2021).

Results: Data extraction and analysis is in progress. The results presentation will include a descriptive overview of the included studies (regarding their design, experimental stimuli, biological and clinical assessments), an illustration of cortisol reactivity to the traumatic stimuli, as summarized across studies, as well as the metaanalytic effect sizes and related measures.

Discussion: This systematic review will provide a comprehensive overview of the existing experimental studies that investigated cortisol reactivity as a prognostic biomarker for PTSD symptom development. It will point out current research practices, summarize existing knowledge, thereby advance our understanding of the biological mechanisms underlying differential PTSD risk, and identify scientific gaps to be addressed by future research.

Further details: The Open Science Framework preregistration of this systematic review can be accessed here: https://osf.io/9yb7s

\section{6}

\section{Experience of maternal childhood maltreatment predicts impairment of postnatal mother-child bonding}

PD Dr. med. Thorsten Mikoteit ${ }^{1}$, Joelle Pais Sava ${ }^{1}$, Prof. Dr. Sibil Tschudin $^{2}$, PD Dr. phil. Serge Brand ${ }^{3}$, Prof. Dr. med. Martin Hatzinger ${ }^{1}$

${ }^{I}$ Psychiatric Services Solothurn And University Of Basel, Solothurn, Switzerland, ${ }^{2}$ University Hospital Basel, Clinics of Gynecology and Obstetrics, Basel, Switzerland, ${ }^{3}$ University Clincs of Psychiatry, University of Basel, Basel, Switzerland

Objectives: Approximately $30 \%$ of women report having experienced maltreatment during their childhood, many of whom developed depression or difficulties in emotion recognition later in life. The aim of this study was to investigate whether impaired mother-child bonding is a consequence of mother's experience of child maltreatment $(\mathrm{CM})$ or if maternal symptoms of psychopathology (e.g. depression or anxiety) that arise from previous experience of abuse and neglect can be hold responsible for this phenomenon. Furthermore, the impact of emotion recognition and emotional intelligence on mother-child bonding are being discussed.

Methods: Thirty-six pregnant women (age: $33.3 \pm 4.4$ years) were recruited. At enrolment ( $\mathrm{t} 0$ : before 32 nd week of gestation) experience of childhood maltreatment was assessed by the German version of the Childhood Trauma Questionnaire Short Form (CTQ-SF). 12 weeks postpartum (t4), mothers were asked to rate their motherchild bonding by the Postpartum Bonding Questionnaire (PBQ). The Edinburgh Postnatal Depression Scale (EPDS) and Hamilton Depression Rating Scale (HAM-D) measured levels of depression at enrolment ( $\mathrm{t} 0), 32 \mathrm{nd}$ week of gestation ( $\mathrm{t} 1), 2$ days postpartum ( $\mathrm{t} 3$ ) and 12 weeks postpartum (t4). Anxiety was assessed by the StateTrait Anxiety Inventory (STAI) at t0, t 1 and $\mathrm{t} 4$. Measures of emotion recognition and emotional intelligence were determined by the Reading the Mind in the Eyes Test (RME) and Emotionale Kompetenz Fragebogen (EKF), respectively, both at t0. Regression and mediation analysis were performed in order to confirm the model.

Results: Direct effects of emotional abuse $(\mathrm{F}=4.699, \mathrm{p}=.045)$, emotional neglect $(\mathrm{F}=9.956, \mathrm{p}=.006)$, physical neglect $(\mathrm{F}=5.388$, $\mathrm{p}=.032)$, experience of inconsistency $(\mathrm{F}=13.642, \mathrm{p}=.002)$ and sexual abuse $(\mathrm{F}=12.247, \mathrm{p}=.003)$ on impaired bonding were found. Furthermore, direct effects of emotional abuse $(\mathrm{F}=5.707, \mathrm{p}=.029)$, emotional neglect $(\mathrm{F}=5.945, \mathrm{p}=.026)$, physical neglect $(\mathrm{F}=9.234$, $\mathrm{p}=.007)$, experience of inconsistency $(\mathrm{F}=12.616, \mathrm{p}=.002)$ and sexual abuse $(\mathrm{F}=13.105, \mathrm{p}=.002)$ on rejection and anger towards the child could have been detected. Direct effect of experience of inconsistency on anxiety about care was found $(\mathrm{F}=7.735, \mathrm{p}=.012)$. Neither emotion recognition nor emotional intelligence were related to the experience of childhood maltreatment $(\mathrm{F}=3.950, \mathrm{p}=.058$, resp. $\mathrm{F}=1.529, \mathrm{p}=.228$ ) or had an impact on mother-child bonding $(\mathrm{F}=2.919, \mathrm{p}=.106$, resp. $\mathrm{F}=.757, \mathrm{p}=.396)$. Mediation analysis revealed no indirect effect of maternal depressive or anxious symptoms on mother-child bonding overall.

Conclusion: In our sample it could have been shown that the experience of $\mathrm{CM}$ has rather direct than indirect adverse effects on the mother-child relationship quality. Therefore it should be concluded that an intervention would need to aim at both, trauma-related as well as depression therapy, whereas it remains unclear from the current sample size if symptoms of depression potentially affect bonding, too. There was no evidence for improved mother-child bonding when mothers showed higher levels of emotion recognition and emotional intelligence. Moreover, unlike hypothesized, experience of $\mathrm{CM}$ did not predict disrupted emotion recognition nor lower emotional intelligence.

\section{7}

\section{Psychophysiological factors affecting emotion recognition}

Stephanie J. Dimitroff ${ }^{1}$, Bernadette Denk ${ }^{1,2}$, Annika B. E. Benz ${ }^{1}$, Ulrike U. Bentele $^{1}$, Maria Meier ${ }^{1}$, Eva Unternaehrer ${ }^{1,3}$, Jens C. Pruessner ${ }^{1,2}$

${ }^{1}$ Department of Psychology, University Of Konstanz, Konstanz, Germany, ${ }^{2}$ Centre for the Advanced Study of Collective Behaviour, University of Konstanz, Konstanz, Germany, ${ }^{3}$ Child- and Adolescent Research Department, Psychiatric University Hospitals Basel (UPK), University of Basel, Basel, Switzerland

Across most models of emotions, our ability to recognize emotions in others is thought to be crucial for successfully navigating social environments, and for maintaining and forming social relationships. Emotion recognition is a complex phenomenon which is highly variable between individuals. According to the somatovisceral afferent model of emotion (SAME), recognition of emotions in others depends not only on external inputs, but also internal physiological processes, which are variable between individuals. Furthermore, psychosocial variables, like loneliness, and social support are thought to affect how we process emotional inputs. We designed three studies to investigate how various key psychosocial and physiological variables affect emotion recognition (ER) abilities. All studies used the Emotion Recognition Task (ERT). In study one, 40 women were recruited, half of which were taking oral contraceptives (OCs), while the other half was not. We found that OC-users performed significantly worse on the ERT. In study two, an online study, we asked participants $(n=207)$ about their living situations before and during the COVID-19 pandemic, and how many hours per day they spent time interacting with others in person, via video or on the phone. Living situation significantly affected ERT performance, in that individuals who reported living with family members prior to the COVID-19 pandemic performed the worst. No differences were found across subjects either living with romantic partners, with friends, or alone. Furthermore, amount of time spent interacting with others, independent of medium, did not predict ERT scores. Finally, in study three, 50 participants completed an interoceptive accuracy task by counting heartbeats, as well as a social network size questionnaire. 
Interoceptive accuracy of participants was not predictive of ERT scores, and neither was the size of their social networks. Altogether, these results indicate that psychosocial factors like size of social network, and time spent interacting with others, seem to have little effect on ER. Interoceptive accuracy, which according to the SAME model, should improve ER, also had no relationship to accuracy on the ERT in this study. In contrast, the findings from OC users add to building evidence that hormonal changes due to oral contraceptive intake negatively impact ER. Given the importance of ER in social relationships, further research is necessary to establish which physiological and psychosocial factors are related to ER, as such work can eventually help determine how to increase ER abilities in individuals, and thus improve their social outcomes.

\section{8}

\section{Mice deficient for sialyltransferase ST3GAL5 exhibit a spectrum of ADHD/ASD-like behaviours}

Ekaterina Veniaminova ${ }^{1}$, Evgeniy Svirin ${ }^{1,2,3}$, Tatyana Veremeyko ${ }^{4}$, Amanda W.Y. Yung ${ }^{4}$, Daniel Anthony ${ }^{1,5}$, Lee Wei Lim ${ }^{6}$, Susanne Walitza $^{7}$, Klaus-Peter Lesch ${ }^{1,2,8}$, Tatyana Strekalova ${ }^{1,2,3}$, Eugene D. Ponomarev6,9

${ }^{1}$ I.M. Sechenov First Moscow State Medical University, Moscow, Russia, ${ }^{2}$ Maastricht University, Maastricht, Netherlands, ${ }^{3}$ Institute of General Pathology and Pathophysiology, Moscow, Russia, ${ }^{4}$ the Chinese University of Hong Kong, Shatin, Hong Kong, ${ }^{5}$ Oxford University, Oxford, United Kingdom, ${ }^{6}$ University of Hong Kong, Hong Kong, ${ }^{7}$ the University of Zurich and the University Hospital of Psychiatry Zurich, Zurich, Switzerland, ${ }^{8}$ University of Würzburg, Würzburg, Germany, ${ }^{9}$ Kunmin Institute of Zoology, Chinese University of Hong Kong Joint Laboratory of Bioresources and Molecular Research of Common Diseases, Kunmin-Hong Kong, China

Genetic lack of lactosylceramide-alpha-2,3-sialyltransferase (ST3GAL5) in humans leads to severe neuropathology owing to the loss of production of the ganglioside GM3. ST3Gal5 knock-out (st3gal5 - / -) mice lack all a- and b-series gangliosides, which results in LacCer accumulation and a compensatory increase of o-series gangliosides. Surprisingly the St3gal5 - / - mice exhibit no overt neurological phenotype, other than deafness. Further testing has revealed that these mutants are hyperactive, but no further characterisation of ADHD associated behaviours was reported. Here, st3gal5 - / - mice were investigated for endophenotypes of ADHD / ASD (for behavioural signs of hyperactivity, cognitive deficits, compromised social behaviour, and anxiety/depression like changes), and ST3GAL5-related human neuropathology (myelin abnormalities and altered insulin signalling and EEG activity). Mutants of both sexes exhibited anxiety-like behaviour, altered sociability, motor abnormalities and impaired conditioning of taste aversion; males displayed aggression and hyperlocomotion. St3gal5 - / - mice showed increased amplitude of EEG peaks, decreased gene and protein expression of proteolipid protein-1 (Plp1), a major myelin component. As GM3 gangliosides regulate neuroinflammatory mechanisms, the expression of pro-inflammatory cytokines was examined in the brain and spleen, also under conditions of systemic inflammation. Interleukin (IL)-1 $\beta$ expression, in particular, was upregulated in the spleen and, most markedly, in the brain of all mutants. Altered spleen expression of insulin receptor isoforms A and $\mathrm{B}$ was found in st3gal5 $-/-$ mice. Thus, st3gal5 $-/-$ mice exhibit a spectrum of ADHD/ASD-like behaviours, and, as such, provide a useful platform on which to better understand the contribution of the a- and b-series gangliosides to neuronal function and behaviour.

\section{9}

Predation stress causes excessive aggression in female mice with partial genetic inactivation of tryptophan hydroxylase-2: neurochemical and behavioral correlates

Evgeniy Svirin $^{1,2,3}$, Dr. Ekaterina Veniaminova ${ }^{1}$, Dr. Joao CostaNunes $^{4}$, Prof. Daniel Anthony ${ }^{5}$, Prof. Lee Wei Lim ${ }^{6}$, Prof. Susanne Walitza $^{7}$, Prof. Klaus-Peter Lesch ${ }^{1,2}$, Prof. Tatyana Strekalova ${ }^{1,2,3}$

${ }^{1}$ Department of Psychiatry and Neuropsychology, School for Mental Health and Neuroscience (MHeNS), Maastricht University, Maastricht, The Netherlands, ${ }^{2}$ Division of Molecular Psychiatry, Center of Mental Health, University of Würzburg, Würzburg, Germany, ${ }^{3}$ Laboratory of Psychiatric Neurobiology, Sechenov First Moscow State Medical University, Moscow, Russian Federation, ${ }^{4}$ Institute of Molecular Medicine, New University of Lisbon, Lisbon, Portugal, ${ }^{5}$ Department of Pharmacology, Oxford University, Oxford, United Kingdom, ${ }^{6}$ School of Biomedical Sciences, Faculty of Medicine, University of Hong Kong, Hong Kong, Hong Kong, ${ }^{7}$ Department for Child and Adolescent Psychiatry and Psychotherapy, University of Zurich, Switzerland

Gene-environment interaction $(\mathrm{G} \times \mathrm{E})$ determines the vulnerability of an individual to a spectrum of stress-related neuropsychiatric disorders. Increased impulsivity, excessive aggression, and other behavioral characteristics are associated with variants within the tryptophan hydroxylase-2 (Tph2) gene, a key enzyme in brain serotonin synthesis. This phenotype is recapitulated in naïve mice with complete, but not with partial Tph2 inactivation. Tph2 haploinsufficiency in animals reflects the allelic variation of $\mathrm{Tph} 2$ facilitating the elucidation of respective $\mathrm{G} \times \mathrm{E}$ mechanisms. Recently, we showed excessive aggression and altered monoamine brain metabolism in heterozygous Tph2-deficient male and female mice $\left(\mathrm{Tph} 2+I_{-}\right)$ after predator stress exposure. Predation stress procedure increases measures of aggression, dominancy, and suppresses sociability in $\mathrm{Tph} 2+/-$ mice, which was the opposite of that observed in control mice. Stressed mutants displayed altered gene expression of 5-HT receptors, markers of stress and inflammation, as well as several myelination-related molecules: myelin basic protein (Mbp), proteolipid protein 1 (Plp1), myelin-associated glycoprotein (Mag), and myelin oligodendrocyte glycoprotein (Mog). Naïve Tph $2+/-$ mice exposed to environmental novelty or to the ModFST exhibited increased novelty exploration and no increase in floating behavior compared to controls, which is suggestive of resilience to stress and despair. In conclusion, environmentally challenged Tph $2+/-$ mice exhibit behaviors that resemble the behavior of non-stressed null mutants, which reveals how $\mathrm{G} \times \mathrm{E}$ interaction studies can unmask latent genetically determined predispositions.

\section{0}

HPA axis activity in the course of the menstrual cycle a meta-analysis of longitudinal studies

Hannah Klusmann ${ }^{1}$, Dr. Lars Schulze ${ }^{1}$, Dr. Sinha Engel ${ }^{1}$, Prof. Dr. Christine Knaevelsrud ${ }^{1}$, Prof. Dr. Sarah Schumacher ${ }^{2}$

${ }^{1}$ Freie Universität Berlin, Berlin, Germany, ${ }^{2} \mathrm{HMU}$ Health and Medical University Potsdam, Potsdam, Germany 
Background: Due to its influence on behavior and cognition, the hypothalamic-pituitary-adrenal (HPA) axis plays an important role in psychological stress-related disorders, such as depression or posttraumatic stress disorder. Many of these disorders show sex-specific differences in prevalence and severity. Women appear to be at increased risk during phases of increased sex steroid fluctuations, such as puberty, postpartum or menopause. This suggests that changes in gonadal hormones, which are regulated by the hypothalamic-pituitary-gonadal (HPG) axis, interact with cortisol, the end product of the HPA axis. Therefore, the interaction of the HPA axis and the HPG axis might inform about important determinants in the development and maintenance of stress-related disorders.

In addition to periods of the mentioned severe hormonal fluctuation, gonadal hormones also fluctuate during the menstrual cycle. To what extend these fine-tuned fluctuations between different cycle phases influence the HPA axis is not fully understood. The aim of this metaanalysis is to investigate possible changes in basal cortisol concentrations as a marker for HPA axis activity in relation to menstrual cycle phases.

Methods: A systematic literature search was performed in six databases (PsycINFO, Pubpsych, PsycARTICLES, PubMed, Web of Science, ProQuest Dissertations and Theses Global). Published and unpublished studies were additionally searched for in the grey literature. Because the menstrual cycle is an intraindividual process and hormonal fluctuations differ not only interindividually (between cycle phases) but also intraindividually (between women), only longitudinal studies that assessed cortisol in at least two different cycle phases (within-subjects design) were included.

Inclusion criteria for the studied samples were female sex and a mean age between 18 and 45 years. Exclusion criteria for the samples were pregnancy, breastfeeding, the post-partum phase or peri- and postmenopause. Only healthy samples were included and therefore, somatic diseases and samples with psychological disorders were excluded.

We included cortisol measured in blood, saliva or urine, which reflect daily circadian rhythms (only blood and saliva) and changes in the finer grained regulation of the HPA axis. All available parameters of cortisol (AUC, CAR, single measures, diurnal means, 24-h means) were extracted. We plan to meta-analytically summarize differences in cortisol concentrations between the luteal and follicular phase and additionally, pairwise between five finer grained cycle phases: the menstrual phase, the mid- to late follicular phase, the ovulatory phase, the early- to midluteal phase and the premenstrual phase. The standardized mean change will be used as meta-analytic effect measure of these comparisons. Quality assessments of cortisol and menstrual cycle parameters will be performed.

Results/discussion: 121 studies were included in the meta-analysis. The data analysis is ongoing. The results aim to inform decisions on how to incorporate the menstrual cycle in cortisol research. Furthermore, they will provide a basis for future studies regarding the interaction of the HPA and HPG axes and their possible role in stressrelated disorders. The results will be presented and discussed at the conference.

\section{1}

\section{Nx4 attenuated stress-induced activity of the anterior cingulate cortex: a post-hoc analysis of a placebo- controlled cross-over trial}

Tara Chand ${ }^{1,2}$, Luisa Herrmann ${ }^{1,2}$, Vanessa Kasties ${ }^{1}$, Cindy Boden ${ }^{1}$, Meng $\mathrm{Li}^{2}$, Yan $\mathrm{Fan}^{3}$, Johan Van der Meer ${ }^{4}$, Johannes C. Vester ${ }^{5}$, Bernd Seilheimer ${ }^{6}$, Myron Schultz ${ }^{6}$, Sarah Alizadeh ${ }^{1}$, Martin Walter ${ }^{1,2,7,8}$
${ }^{I}$ Department of Psychiatry and Psychotherapy, Jena University Hospital, Jena, Germany, ${ }^{2}$ Department of Psychiatry and Psychotherapy, University of Tübingen, Tübingen, Germany, ${ }^{3}$ Leibniz Research Centre for Working Environment and Human Factors, Dortmund, Germany, ${ }^{4}$ QIMR Berghofer Medical Research Institute, Brisbane, Australia, ${ }^{5}$ idv Data Analysis and Study Planning, Krailling, Germany, ${ }^{6}$ Biologische Heilmittel Heel GmbH, BadenBaden, Germany, ${ }^{7}$ Medical Faculty, Otto von Guericke University of Magdeburg, Magdeburg, Germany, ${ }^{8}$ Leibniz Institute for Neurobiology, Department of Behavioral Neurology, Magdeburg, Germany

Background: Stress-related symptoms are associated with significant health and economic burden. The chronic exposure to stressors is linked to the occurrence of mental disorders such as anxiety and depression. Neurexan ( $\mathrm{Nx} 4)$, a natural multicomponent medicinal product, was shown to have a beneficial effect on reducing stressrelated symptoms such as nervousness and restlessness (Hubner et al. 2009) and also decreasing the neuroendocrine response to an acute stressor (Doering et al. 2009). Here, we hypothesized that the stressrelieving properties of $\mathrm{Nx} 4$ are based on a direct effect on neural processing linked to a predefined stress network which has been associated with a variety of stress-related symptoms in patients. More specifically, we hypothesized that $\mathrm{Nx} 4$ would significantly dampen the effect of acute stressors on the neural stress network, including anterior cingulate cortex (ACC), medial orbitofrontal cortex, hippocampus, amygdala, and hypothalamus compared to placebo.

Methods: In a randomized, placebo-controlled, double-blind, twosequence, cross-over trial (NEURIM study; ClinicalTrials.gov identifier: NCT02602275), 39 mild to moderate stressed healthy males took a single dose (3 tablets) of placebo or $\mathrm{Nx} 4$ before exposure to acute psychosocial stress. The ScanSTRESS paradigm, which uses demanding arithmetic calculations and mental rotation tasks with negative feedback, was used to induce psychosocial stress inside a Philips 3-T MRI scanner (Streit et al., 2014). In total, 225 volumes of echo-planar images were acquired during the ScanSTRESS paradigm. The data were preprocessed with minimal processing, including slicetiming, realignment, co-registration, normalization, and smoothing (8 mm FWHM kernel) steps using SPM-12 and MATLAB-19a. The stress network activation was analyzed by fMRI in brain regions, including ACC, hippocampus, amygdala, medial orbitofrontal cortex defined by the Automated Anatomical Labeling (AAL) Atlas, and hypothalamus identified following prior literature (Kroemer et al. 2013). Five separate regressors were constructed to evaluate the task and drug effect, one for each of the four conditions of interest (control subtraction, control mental rotation, stress subtraction, stress mental rotation) and one for the instruction. For each participant, the contrasts of interest for stress versus control conditions were calculated. Results: Using the placebo data only, significantly higher activations in the anterior insula (bilaterally), premotor area (left), angular gyrus (bilaterally), inferior frontal gyrus (bilaterally), posterior medial frontal gyrus (left), occipital lobe, cerebellum, supplementary motor area and deactivations in the default mode network regions were found when comparing stress and control blocks, which could validate the induction of a distinct neural stress activation pattern by the ScanSTRESS paradigm. For Nx4, we could provide evidence of an attenuating effect on the stress response in this stress network. The rotating stress task of the ScanSTRESS paradigm showed a statistically significant decrease in differential stress-induced activity in the right supracallosal ACC. No significant differential activation was observed for the overall stress task and the subtraction task comparing the placebo and $\mathrm{Nx} 4$ conditions.

Limitations: The effect was localized to a specific brain region in a particular stress task in an exploratory analysis. 
Conclusions: Our findings support the hypothesis that $\mathrm{Nx} 4$ modulates the stress response by lowering activity in the neural stress network, especially in the ACC.

\section{2}

\section{Too nervous to sleep? Novel behavioral aspects of the intracerebroventricular streptozotocin-induced rat model of sporadic Alzheimer's disease}

Jan Homolak ${ }^{1}$, Davor Virag ${ }^{1}$, Ana Babic Perhoc ${ }^{1}$, Ivan Kodvanj ${ }^{1}$, Mihovil Joja ${ }^{1}$, Ana Knezovic ${ }^{1}$, Jelena Osmanovic Barilar ${ }^{1}$, Melita Salkovic-Petrisic ${ }^{1}$

\section{${ }^{1}$ University of Zagreb School Of Medicine, Zagreb, Croatia}

Introduction: Alzheimer's disease (AD) has been described more than 115 years ago, however, its etiopathogenesis remains unknown to date. Consequently, modeling of the disease in laboratory animals remains a challenge and the development of novel treatment solutions have been unsuccessful so far. Cognitive impairment is the most pronounced behavioral alteration present in both patients and animal models of the disease, nevertheless accompanying neuropsychiatric symptoms such as anxiety are often neglected and the behavioral complexity of current $\mathrm{AD}$ models remains largely unexplored. Considering neurobehavioral phenomena provide a unique insight into the pathophysiology of the central nervous system, acknowledgment of subtle behavioral changes may provide additional information indispensable for understanding the development of neuropathology in animal models. The present aim was to explore behavioral alterations accompanying deficits of learning and memory in the widely used intracerebroventricular streptozotocin (STZ-icv)-induced rat model of sporadic AD with a special focus directed to circadian alterations, stress response, and attention/impulsivity recently recognized as neglected behavioral and psychological symptoms often present in the early stage of the disease.

Materials and methods: Independent cohorts of male Wistar rats treated with STZ-icv $(3 \mathrm{mg} / \mathrm{kg})$ and vehicle-treated controls have been studied. Acute and sub-acute circadian changes have been assessed by a custom-made circadian tracker-multi-cage infra-red open-source locomotor activity viewer (MIROSLAV). Acoustic startle and prepulse inhibition sensorimotor gating have been examined using the custom-made griPASTA platform. Open field test, social preference, novel object recognition, and passive avoidance have been used for assessment of general (anxiety, cognition, social preference, recognition memory, and associative learning) and subtle (attention dynamics, hesitancy/impulsivity) behavioral patterns.

Results: Preliminary evidence suggests STZ-icv rats suffer from pronounced circadian dysrhythmia that develops already upon administration of the first dose of STZ-icv. Apart from well-known cognitive deficits, STZ-icv rats suffer from increased acoustic startle response with unaltered prepulse inhibition sensorimotor gating, increased anxiety accompanied by paradoxically increased exploration drive, and increased social preference. Furthermore, analysis of subtle behavioral patterns uncovers increased attention shifting and impulsivity in the STZ-icv rats.

Conclusion: Deficits of learning and memory are well-known behavioral alterations present in the STZ-icv rats. Nevertheless, thorough exploration of the behavioral phenotype uncovers a complex pattern characterized by overlapping neurobehavioral phenomena that might provide a unique insight into the etiopathogenesis of STZ-icvinduced neurodegeneration with anxiety, altered stress response, and circadian dysrhythmia indicating the potential importance of the hypothalamus. Furthermore, acknowledgment of the behavioral complexity may uncover potential sources of bias and significantly contribute to the robustness and reliability of the results from the experiments exploiting the STZ-icv rat model of AD.

This work was funded by the Croatian Science Foundation (IP-201801-8938). Research was co-financed by the Scientific Centre of Excellence for Basic, Clinical and Translational Neuroscience (project "Experimental and clinical research of hypoxic-ischemic damage in perinatal and adult brain"; GA KK01.1.1.01.0007 funded by the European Union through the European Regional Development Fund).

\section{3}

\section{$\mathrm{Nx} 4$ attenuates stress-induced brain activity patterns in anxiety-prone individuals}

Melanni Nanni-Zepeda ${ }^{1,2}$, Sarah Alizadeh $^{1}$, Tara Chand ${ }^{1,2}$, Vanessa Kasties ${ }^{1}$, Yan Fan $^{3}$, Johannes Vester ${ }^{4}$, Bernd Seilheimer ${ }^{5}$, Myron Schultz $^{5}$, Luisa Herrmann ${ }^{1}$, Martin Walter ${ }^{1,2}$

${ }^{1}$ University of Tübingen, Department of Psychiatry and Psychotherapy, Division for Translational Psychiatry, Tuebingen, Germany, ${ }^{2}$ Jena University Hospital, Department of Psychiatry and Psychotherapy, Jena, Germany, ${ }^{3}$ Leibniz Research Centre for Working Environment and Human Factors, Germany, ${ }^{4} \mathrm{idv}$ Data Analysis and Study Planning, Krailling, Germany, ${ }^{5}$ Biologische Heilmittel Heel GmbH, Baden-Baden, Germany

Background: One of the most relevant characteristics contributing to shape the stress response is anxiety as a personality trait (TA). It is well known that the amygdala and its widespread cortical and subcortical connections play an important role in psychosocial stress and anxiety. Previous research suggested that Neurexan $(\mathrm{Nx} 4)$ attenuates the neuroendocrine stress response, alleviates stress-related symptoms, and alters stress reactivity in healthy volunteers. In this study we investigated how the inter-individual variability in TA influences the response to $\mathrm{Nx} 4$ treatment. We measured stress-induced changes in amygdala-centered resting-state functional connectivity (rsFC) in mildly to moderately stressed healthy volunteers.

Methods: In a randomized, placebo-controlled, double-blind, twosequence, cross-over trial (NEURIM study; ClinicalTrials.gov identifier: NCT02602275), 39 healthy males experiencing mildly to moderately chronic stress took a single dose (3 tablets) of either placebo or $\mathrm{Nx} 4$ before exposure to acute psychosocial stress, which was induced using the ScanSTRESS paradigm inside a Philips 3-T MRI scanner. The oral intake took place 60-80 min before the fMRI scan, which consisted of the ScanSTRESS paradigm as well as 12 min pre-stress (RS1) and post-stress (RS2) resting-state scans. Trait anxiety was assessed through the State-Trait Anxiety Inventory (STAI), approximately 3-7 days before the first experimental day. $\mathrm{rsFC}$ analysis was centered around the left and right amygdala as seed regions by the Automated Anatomical Labeling atlas. Six subjects were excluded due to excessive motion artifacts, resulting in 33 subjects. To build the regression model, we first calculated $\mathrm{Nx} 4$ efficacy measure as Verum(FCRS2 -FCRS1)-Placebo(FCRS2FCRS1), which represents the drug effect on stress-induced rsFC changes from the seed (left or right amygdala) to any region in the brain. Next, correlations between $\mathrm{Nx} 4$ 's efficacy and trait anxiety scores were calculated with SPM by adding it as a covariate, as well as age and drug sequence.

Results: Regression analysis revealed that Nx4's effect on stressinduced changes in rsFC between right amygdala (rAmg) and prefrontal areas, namely the perigenual anterior cingulate cortex (pgACC) and ventro-medial prefrontal cortex (vmPFC) is modulated by TA $(r=-0.716 ; p<3 E-06)$. Higher individual scores of TA are associated with reduced stress-induced changes in verum compared to placebo, indicating stronger $\mathrm{Nx} 4$ efficacy on subjects with more 
pronounced TA. In participants with higher TA (compared to the normative value STAI $>34.89$ ), the rAmg-pgACC/vmPFC rsFC increases significantly after stress for placebo $(p=0.040)$ whereas it decreases significantly in verum condition $(p=0.016)$. A significant $\mathrm{Nx} 4$ effect on Amg-prefrontal rsFC changes was only found in the group of subjects who scored higher in TA $(p<0.004)$.

Conclusion: Results suggest that the effect of $\mathrm{Nx} 4$ on stress-induced changes in rsFC between rAmyg, and pgACC-vmPFC varies depending on TA levels. This connectivity pattern reflected a higher amygdala-prefrontal rsFC level in individuals with higher TA, in the placebo condition after stress induction. In the $\mathrm{Nx} 4$ condition, there was a decrease in the Amg-prefrontal rsFC, suggesting a modulatory role of $\mathrm{Nx} 4$ in reducing the stress response in subjects with high TA. This adds further evidence to the stress reducing effects of $\mathrm{Nx} 4$ in different populations from other studies.

\section{4}

\section{Antagonistic effects of neuropeptide $Y$ and corticotropin-releasing factor on glutamatergic synaptic transmission in corticotropin-releasing factor- expressing neurons of the bed nucleus of the stria terminalis}

Dr. med. Julia Constance Bartsch ${ }^{1}$, Robert Schumacher ${ }^{1}$, Prof. Dr. rer. nat. Hans-Christian Pape ${ }^{1}$, PD Dr. rer. nat. Kay Jüngling ${ }^{1}$

${ }^{1}$ Westfälische Wilhelms-Universität Münster, Münster, Germany

The anterior bed nucleus of the stria terminalis (BNST) and the endogenous neuropeptide systems of corticotropin-releasing factor (CRF) and neuropeptide Y (NPY) contribute to shaping stress and anxiety responses. To date, largely antagonistic behavioral effects of NPY and CRF on stress and anxiety have been reported. The BNST also contains NPY, CRF and their specific G-protein-coupled receptors. So far, a bidirectional modulation of inhibitory synaptic transmission in BNST by NPY and CRF at the Y2 receptor (Y2R) and the CRF receptor 1 (CRFR1) has been shown. Here, we used wholecell patch-clamp recordings in acute slices of anterior dorsal BNST from transgenic CRF reporter mice to characterize the impact of both neuropeptides on excitatory synaptic activity. While selective pharmacological activation of $\mathrm{Y} 2 \mathrm{R}$ inhibited glutamatergic synaptic transmission in both CRF-positive and CRF-negative neurons via a presynaptic mechanism, selective activation of CRFR1 preferentially enhanced glutamatergic synaptic transmission in CRF-positive neurons, likely through a postsynaptic mechanism. Taken together, our results suggest potential anatomical and cellular substrates for a functional antagonism of NPY and CRF in the anterior dorsal BNST. Thus, BNST outputs from CRF-expressing neurons in this critical hub could form a distinct part of an anxiety- and stress-modulating circuitry governed by NPY- and CRF-driven neuromodulation.

\section{5}

\section{The effect of fatigue on HPA axis functioning in chronically stressed individuals}

\author{
Dr. Nida Ali ${ }^{1}$, Dr. Nadine Skoluda ${ }^{1}$, Dr. Jana Strahler ${ }^{1}$, Dr. Urs \\ Nater $^{1}$ \\ ${ }^{1}$ University Of Vienna, Vienna, Austria
}

Background: Individuals suffering from chronic fatigue have been found to experience significantly more stress compared to healthy individuals. However, not everyone experiencing chronic levels of stress becomes fatigued, indicating that inherent individual differences exist in stress sensitivity and risk for fatigue. While both chronically stressed and fatigued patients show hypoactivity of the hypothalamic-pituitary-adrenal (HPA) axis, it is not known whether differential patterns of HPA axis dysfunction exist in chronically stressed individuals with varying levels of fatigue.

Methods: 61 chronically stressed (CS; $38.31 \pm 14$ years) and 55 low-stress (LS; $37.90 \pm 14.37$ years) women were exposed to a psychosocial laboratory stressor. Salivary cortisol measures were assessed throughout the study. Fatigue was assessed using the Multidimensional Fatigue Inventory.

Results: The results revealed overall that CS had significantly lower cortisol levels and higher fatigue compared to LS (both $\mathrm{p}<.05$ ). Within the CS group, increasing levels of fatigue were not associated with changes in cortisol. In the LS group, however, there was an inverse relationship between fatigue and cortisol, with increasing levels of fatigue associated with lower cortisol $(\mathrm{p}<.05)$.

Conclusions and implications: Our findings indicate that in the CS group, stress-related hypoactivity of the HPA axis may have resulted in a floor effect for cortisol, such that increasing levels of fatigue, in addition to existing chronic stress, did not have cumulative effects on the already blunted HPA axis. This was in contrast to the LS group, where fatigue had important effects on HPA axis functioning, such that decreases in cortisol were observed as fatigue levels increased.

\section{6}

\section{A laboratory model of human exposure therapy in posttraumatic stress disorder: temporal considerations and brain mechanisms}

Pedro Correia ${ }^{1,2}$, Kornél Demeter ${ }^{1,3}$, János $\operatorname{Varga}^{1}$, Eszter Urbán ${ }^{1}$, Bibiána Török ${ }^{1,2}$, Diána Balázsfi ${ }^{1}$, Nikolett Bakos ${ }^{1}$, József Haller ${ }^{1}$, Dóra Zelena ${ }^{1,4}$

${ }^{1}$ Department of Behavioral Neurobiology, Institute of Experimental Medicine, Budapest, Hungary, ${ }^{2}$ Janos Szentagothai School of Neurosciences, Semmelweis University, Budapest, Hungary, Budapest, Hungary, ${ }^{3}$ Behavioral Studies Unit, Institute of Experimental Medicine, Budapest, Hungary, Budapest, Hungary, ${ }^{4}$ Centre for Neuroscience, Szentágothai Research Centre, Institute of Physiology, Medical School, University of Pécs, Pécs, Hungary, Pécs, Hungary

Posttraumatic stress disorder (PTSD) is a serious mental disorder developing after traumatic events. Unfortunately, its prevalence is increasing worldwide, especially due to COVID-19 pandemic. Exposure therapy is one of the treatment options, which can be combined with pharmacological treatment. Testing efficacy requires preclinical animal models. However, there are no established protocols and the efficacy of particular protocols as well as the underlying brain mechanisms remain also unknown.

The present study examined three different extinction protocols with various starting time (one or twenty-eight days after trauma) and investigated the efficacy as well as the durability of the fear extinction. Male Wistar rats subjected to inescapable electric foot shocks were used as animal models. Behavior (freezing in trauma-context) and stress hormone levels (adrenocorticotropin and corticosterone in serum) were followed during extinction. Subsequent to the termination of the extinction training a c-Fos immunohistochemistry was performed to identify the neuronal activation patterns. We were focusing on three brain areas implicated in PTSD (prefrontal cortex (PFC), hippocampus (HC) and amygdala). 
A spontaneous recovery of extinguished recent (starting 1 day after trauma) fear memory was detected three weeks after training. Opposite to that, the extinction of remote fear memory (extinction started 28 days after trauma) was significantly more long lasting, as no recovery was observed at the same period. Hormone measurements performed at the end of extinction trials suggest that adrenocorticotropin, but not corticosterone responses resemble behavioral extinction without any sign of relapse. Furthermore, after the extinction of recent fear memories, re-exposure to the conditioning cage increased the activity of the mPFC, and decreased the activation of the central and medial amygdala as compared to rats that were not exposed to extinction training. In this specific situation, no differences were observed in the $\mathrm{HC}$ activity. However, in the case of remote conditioned fear, extinction decreased fear-induced neuronal activation in the $\mathrm{HC}$ and basolateral amygdala, but no major effects were observed in the $\mathrm{mPFC}$.

These findings demonstrate that the extinction of remote fear memories is more effective than that of recent ones, possibly because of the temporal changes in the neuronal networks underlying fear expression.

67

\section{Fear training induces neuropeptide $Y$ receptor type 2-dependent metaplasticity in local inhibitory synaptic networks of anteroventral bed nucleus of the stria terminalis}

Dr. med. Julia Constance Bartsch ${ }^{1}$, PhD Sara Jamil ${ }^{1}$, Dr. rer. nat. Dilip Verma ${ }^{1}$, Prof. Dr. rer. nat. Hans-Christian Pape ${ }^{1}$

\section{${ }^{1}$ Westfälische Wilhelms-Universität Münster, Münster, Germany}

Recently, neuropeptide $\mathrm{Y}$ receptors type 2 (Y2R) within local GABAergic networks in anteroventral bed nucleus of the stria terminalis (BNSTav) have been revealed as key elements in facilitating extinction and reducing return of remote fear memories. Here, we combined fear training and behavioral studies in vivo with electrophysiological analysis of BNSTav synaptic plasticity ex vivo, to identify potentially Y2R-regulated fear-induced synaptic plasticity within these local GABAergic circuits. We chose to investigate two time points of fear training: $24 \mathrm{~h}$ after fear acquisition (potentially related to early fear memory) and 14 days after extinction training (potentially related to remote fear memory). Basal stimulus-induced synaptic transmission did not differ between control animals (handling only) and fear-trained animals ( $24 \mathrm{~h}$ after fear acquisition and $14 \mathrm{~h}$ after extinction training). Only fear acquisition induced Y2Rdependent alterations in short-term synaptic plasticity within GABAergic BNSTav circuits. Concerning potential fear-induced changes in long-term synaptic plasticity, high-frequent stimulation elicited long-term depression (LTD) in fear-trained animals (24 h after fear acquisition and $14 \mathrm{~h}$ after extinction training) by shifting the response frequency distribution towards LTD compared to control animals. Bath-application of a specific Y2R antagonist in slices from fear-trained mice prevented LTD of inhibitory transmission in BNSTav neurons ( $24 \mathrm{~h}$ after fear acquisition and $14 \mathrm{~h}$ after extinction training) and changed back response frequency distribution upon high-frequency stimulation after fear acquisition, indicating a Y2Rdependent shift towards inhibitory LTD in BNSTav circuits. Together our data suggest that GABAergic BNSTav circuits might contribute to encoding of fear memory through Y2R-modulated shift in inhibitory synaptic plasticity at both early and remote time points after fear conditioning. Notably, unmasking a Y2R-modulated alteration of long-term synaptic plasticity selectively by a high-frequency stimulation protocol is well in line with the idea of neuropeptide release requiring higher level of neuronal activity. Our observation of an enhanced GABAergic LTD in BNSTav circuits after stressful fear conditioning can be considered as a form of metaplasticity.

\section{8}

HPA axis and ANS activity in the course of an internetbased psychotherapeutic intervention for depression

Sebastian Laufer ${ }^{1}$, Dr. Sinha Engel ${ }^{1}$, Hannah Klusmann ${ }^{1}$, Dr. Lars Schulze $^{1}$, Dr. Nadine Skoluda ${ }^{2}$, Prof. Dr. Urs Nater ${ }^{2}$, Prof. Dr. Christine Knaevelsrud $^{1}$, Prof. Dr. Sarah Schumacher ${ }^{3}$

${ }^{1}$ Freie Universität Berlin, Berlin, Germany, ${ }^{2}$ Universität Wien, Wien, Austria, ${ }^{3}$ Health and Medical University, Potsdam, Germany

Background: Depression has been related to a dysregulation of the biological stress systems, that is, the hypothalamic-pituitary-adrenal (HPA) axis and the autonomic nervous system (ANS). In particular, evidence points towards enhanced basal cortisol concentration as well as higher salivary alpha-amylase concentration in depressed compared to healthy individuals. Successful psychotherapeutic treatment might be accompanied with a re-regulation of the systems. Thus far, only few studies have examined HPA axis or ANS activity over a full course of depression treatment and have usually focused on only one of these systems. The aim of the current study was to examine markers of both stress systems in parallel over the course of an internet-based psychotherapeutic intervention for depression. It was hypothesized that pre- to post-intervention decreases in salivary cortisol and alpha-amylase would be more pronounced in treatment responders as compared to non-responders. Furthermore, responders were expected to have lower cortisol and alpha-amylase levels preintervention compared to non-responders.

Methods: $\mathrm{N}=47$ patients with a major depressive episode underwent a seven-week therapist-guided internet-based cognitive-behavioraltherapy-based intervention for depression. At pre-, mid- and postintervention, participants provided diurnal profiles of saliva samples over two consecutive days, respectively. Furthermore, depressive symptoms were assessed by the Patient Health Questionnaire (PHQ9) and the Beck Depression Inventory (BDI-II) at each time point. Results: The data are currently still analyzed and will be presented and discussed at the conference.

\section{9}

\section{The behavioral role of the GABAergic neurons in the median raphe region}

\author{
Tiago Chaves ${ }^{1,2}$, Bibiána Török ${ }^{1,2}$, Csilla Fazekas ${ }^{1,2}$, Pedro \\ Correia $^{1,2}$, Dr. Eszter Sipos ${ }^{1}$, Dr Dora Zelena ${ }^{1,3}$ \\ ${ }^{1}$ Laboratory of Behavioural and Stress Studies, Institute

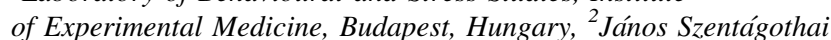 \\ School of Neurosciences, Semmelweis University, Budapest, Hungary, \\ ${ }^{3}$ Centre for Neuroscience, Szentágothai Research Centre, Institute \\ of Physiology, Pécs, Hungary
}

Gamma-aminobutyric acid (GABA) is a well-known inhibitory neurotransmitter implicated in numerous physiological and pathological behavior including social interest and anxiety. Dysregulation of a serotoninergic nucleus, median raphe region (MRR) is also characterized by the rise of social problems. Because the vast majority of the MRR cells are GABAergic our aim was to clarify the behavioral role of these cells. 
We used pharmacogenetic technology in mice containing Cre enzyme under the promoter of the GABA transporter. With the help of an adenoassociated virus, artificial receptors (DREADD, stimulatory, inhibitory as well as control mCherry) were injected into the MRR. Few weeks later they were stimulated by the artificial ligand (clozapine-N-oxide, $1 \mathrm{mg} / 10 \mathrm{ml} / \mathrm{kg}$ ) given $30 \mathrm{~min}$ before the experiments, where social behavior (sociability; social interaction; residentintruder) was studies. As possible confounding factors, locomotion (open field/OF), anxiety (elevated plus maze/EPM), and short-term memory (y-maze) were also evaluated.

Stimulation of the GABAergic cells of the MRR induced no difference in locomotion nor memory, however, it displayed an increased social interested in both sociability and social interaction test. In the EPM test, inhibitory group displayed high latency when entering the open arm, suggesting an anxiolytic-like tendency.

All in all, our experiments confirm the role of MRR GABAergic cells in promoting social interest. We can assume that these cells are not homogenous and different subpopulations (e.g. long vs short projecting, various neuropeptide containing) might have different role in the observed behaviours, which may remain hidden in our experiments. Further studies with more specific subgroups are required in this field.

\section{0}

\section{Perceived stress, anxiety, depression, and the cortisol awakening response: a longitudinal study in young German adults during the COVID-19 pandemic}

\author{
Maria Meier ${ }^{1}$, Hannah Rentschler ${ }^{1}$, Annika B E Benz ${ }^{1}$, Ulrike U \\ Bentele $^{1}$, Stephanie J Dimitroff ${ }^{1}$, Bernadette F Denk ${ }^{1}$, Prof. Dr. Jens \\ C Pruessner ${ }^{1}$, Dr. Eva Unternaehrer ${ }^{2}$ \\ ${ }^{1}$ Department of Psychology, University of Konstanz, Konstanz, \\ Germany, ${ }^{2}$ Child and Adolescent Research Department, Psychiatric \\ University Hospitals Basel, University of Basel, Basel, Switzerland
}

Introduction: The Sars-CoV-2 pandemic resulted in a worldwide health crisis, which has a strong impact on individuals and the society, and unknown long-term effects on psychophysiological health. Chronic stress, anxiety and depressive symptoms have all been linked to dysregulations of the endocrine stress system in previous research. In this study, we monitored perceived stress (PS), anxiety symptoms (AS) and depressive symptoms (DS) during the ongoing Sars-CoV-2 pandemic and investigated how they relate to the cortisol awakening response (CAR), an integral marker of the endocrine stress system. Methods: Using an online survey, we measured PS (Perceived Stress Scale), AS and DS (Brief Symptoms Inventory subscales) in German adults just prior to the onset of the pandemic ( $\mathrm{t} 0=$ autumn 2019), and at three follow-up timepoints $(\mathrm{t} 1=$ spring $2020, \mathrm{t} 2=$ autumn 2020 , $\mathrm{t} 3=$ spring 2021). We measured the CAR by assessing salivary cortisol levels at awakening and $+30,+45,+60$ min thereafter on two consecutive workdays in March/April 2021. The CAR was analyzed in accordance to previously published expert consensus guidelines (Stalder et al., 2016) and operationalized using the area under the curve with respect to increase. Additionally, post-awakening cortisol concentrations were computed using the area under the curve with respect to ground. We hypothesized that PS increased significantly after $\mathrm{t} 0$, and that this increase is linked to an increase in AS and DS. Further, we tested whether PS, AS, DS, and/or dynamic changes thereof predict the CAR at $\mathrm{t} 3$.

Results: A subsample of $\mathrm{N}=33(\mathrm{n}=26$ female, meanage $=26.24$, SDage $=9.46)$ participants completed all components of the study. PS increased by $14.75 \%$ (difference $\mathrm{t} 0-\mathrm{t} \mathrm{t}: \mathrm{t}(32)=-1.37, \mathrm{p}=.090$, $\mathrm{d}=-0.24$ ) and remained heightened throughout $\mathrm{t} 2$ and $\mathrm{t} 3$. AS did not change significantly throughout the assessment (e.g. difference t0-t1: $\mathrm{t}(32)=1.55, \mathrm{p}=.935, \mathrm{~d}=0.27$ ), but DS increased by $20.18 \%$ (difference t0-t1: $\mathrm{t}(32)=-2.34, \mathrm{p}=.013, \mathrm{~d}=-0.41)$ and remained heightened thereafter. PS increase from to to $t 1$ was significantly related to DS increase in the same interval, $\mathrm{r}(31)=.37, \mathrm{p}=.032$, however it was not linked to changes in AS from t0 to $\mathrm{t} 1, \mathrm{r}(31)=.33$, $\mathrm{p}=.061$. Higher average levels of PS at $\mathrm{t} 0$ and $\mathrm{t} 1$ predicted higher average levels of AS $(b=0.19, \mathrm{SE}=0.00, \mathrm{t}(31)=3.10, \mathrm{p}<.001)$ and DS $(b=0.38, S E=0.10, t(31)=3.74, p<.001)$ at timepoints $\mathrm{t} 2$ and $\mathrm{t} 3$. Changes in DS over time were explained by changes in PS in a multilevel mixed model. Finally, higher PS at t1 and higher PS levels over the course of the pandemic (mean t 1 to 3 ) predicted higher postawakening cortisol concentrations, 95\%CI [13.03; 42.80], [1.24; 29.97], [12.88; 52.29], but not the CAR.

Discussion: Our results show an increase in depressive symptoms after the onset of the Sars-Cov-2 pandemic. Higher chronic stress levels at the beginning of the pandemic were prospectively related to more depressive and anxiety symptoms. We found partial evidence for a relation between chronic stress and the regulation of the endocrine stress axis. Due to a lack of statistical power and the young, well-educated sample, our results need to be interpreted with caution. Overall, our findings illustrate the impact of chronic stress on mental health, and depressive symptoms in particular.

\section{1}

\section{How depressive symptoms in targets can influence perceivers' empathic accuracy-importance of emotional valence}

Jonas P. Nitschke ${ }^{1}$, Amy J.P. Gregory ${ }^{1}$, Dr. Melanie Dirks ${ }^{1}$, Dr. Lauren Human $^{1}$, Dr. Jennifer A. Bartz ${ }^{1}$

${ }^{1}$ Mcgill University, Montreal, Canada

Background: Depression has been linked to many interpersonal problems-in part due to difficulties in perceiving and understanding emotions of oneself and others. Indeed, depressive symptoms have been associated with lower levels of understanding the cause and course of one's own emotions, and have also been associated with decreased empathic abilities. In addition, depression have also been shown to inhibit emotion expression, thus impacting how accurately others (i.e., perceivers) can read the depressed individual's (i.e., target's) emotions.

Aim: Here, we aimed to examine how depressive symptoms of targets influence perceivers' ability to understand and accurately track those targets' emotions over time-or empathic accuracy. We predicted that higher levels of depressive symptoms exhibited by the targets while they recall autobiographical events, would impede the perceivers' empathic accuracy for the targets' emotions.

Method: Targets completed a depression measure before being videotaped describing emotionally salient autobiographical events $(n=75)$. Targets rated their own emotion valence throughout each video. Perceivers then watched those videos and rated the targets' emotional valence. Empathic accuracy scores were created for each video by calculating the correlation between targets' own ratings of their affect and perceivers' ratings of the target's emotions.

Results: Mixed-effects model analyses found that perceivers demonstrated higher empathic accuracy when rating positively valenced videos compared to negatively valenced videos. However, targets' depression moderated this link, such that perceivers had lower empathic accuracy when watching depressed targets describe positively-valenced events.

Summary: Our results show that targets' depression influences perceiver empathic accuracy for positively but not negatively valenced 
emotional events. It is possible that depressed targets may simply convey less positive affect. Alternatively, depressed targets may have a skewed perception of their own positive emotions and not subjectively report the positive affect they appear to express. This work provides insights into the interpersonal consequences of depression, specifically how perceivers experience and understand depressed individuals.

\section{2}

\section{Identification and characterisation of neuronal populations specifically activated by emotional stressors in the medial prefrontal cortex}

Patricia Molina $^{1,2}$, Xavier Belda ${ }^{1,2}$, Silvia Fuentes ${ }^{1,3}$, Humberto Gagliano $^{1,2}$, Roser Nadal ${ }^{1,3}$, Raül Andero ${ }^{1,3}$, Antonio Armario ${ }^{1,2}$

${ }^{1}$ Institut de Neurociències, Universitat Autònoma De Barcelona, Barcelona, Spain, ${ }^{2}$ Departament de Biologia Cel-lular, Fisiologia $i$ Immunologia, Universitat Autònoma De Barcelona, Barcelona, Spain, ${ }^{3}$ Departament de Psicobiologia i Metodologia de les Ciències de la Salut, Universitat Autònoma De Barcelona, Barcelona, Spain

Exposure to stress can contribute to the development of psychiatric disorders, but its consequences may differ depending on the characteristics of stressors. Unfortunately, little is known about how stressor aspects impact their processing in the brain. The medial prefrontal cortex (mPFC) has been shown to play a key role in processing emotional stressors and regulating responses to these stimuli, but experimental results are controversial. In this line, our aims are to identify neuronal populations in the $\mathrm{mPFC}$ specifically activated by different emotional stressors and to manipulate them to elucidate their role in the regulation of stress response.

To identify stressor-specific neurons, we have performed double in situ hybridization in rats exposed to two sequential stressors (immobilization, IMO, and footshock, FS) based on the dynamics of expression of the intronic and mature c-fos RNA. We have found that distinct emotional stressors might recruit overlapping but partially distinct neuronal ensembles in the mPFC. We are interested in further characterizing these neuronal populations and for this aim, we are using a phosphoRiboTrap method that allows the molecular profiling of activated neurons based on the phosphorylation of ribosomal protein S6 (pS6). We have already shown that animals exposed to $1 \mathrm{~h}$ IMO show significantly more pS6 + neurons and higher pS6 levels than basal animals, and that there is a high colocalization between pS6 and Fos. Further experiments with this approach will enable us to perform RNA-sequencing of activated neurons and identify the molecular signatures of different stressors.

Furthermore, we have designed a viral vector expressing either the excitatory or inhibitory DREADD under control of the activity-dependent promoter c-fos, which allows us to reactivate or inhibit stressactivated mPFC neurons. Firstly, we have validated that the vector is appropriately induced $4 \mathrm{~h}$ after FS. Secondly, behavioural experiments have been conducted with both vectors. Animals were exposed to FS and $4 \mathrm{~h}$ later to open field (OF) with an object in the centre to evaluate activity and exploration, and one week later, re-exposed to FS and then to forced swim test (FST) to evaluate coping strategies. In both cohorts, FS-exposed animals showed hypoactivity and less object interaction in the OF than controls. With the excitatory DREADD, CNO did not have effects, but with the inhibitory DREADD, CNO increased object interaction time in basal animals. Moreover, in the excitatory cohort, $\mathrm{CNO}$ reduced corticosterone (CORT) levels in both control and stress groups, while with the inhibitory vector there was only an effect of stress, reducing CORT response to OF. Regarding FST, in the excitatory cohort, FS animals injected with vehicle showed less immobility than controls, and remarkably, this effect was reverted by CNO. In terms of the hormonal response to stress, $\mathrm{CNO}$ reduced CORT levels in both control and stress groups. In contrast, no effects of stress or CNO were observed in coping strategies in the inhibitory cohort. Moreover, FSexposed animals showed a lower CORT response to the FST. Further neuronal manipulation experiments are needed to improve our understanding of the role of the $\mathrm{mPFC}$ in the regulation of the stress response.

\section{3}

\section{Prevention of anxiety disorders}

\author{
Prof. Dr. Dr. Katharina Domschke ${ }^{1}$ \\ ${ }^{I}$ Department of Psychiatry, University of Freiburg, Freiburg, \\ Germany
}

Anxiety disorders constitute the most common mental disorders with a 12-month global prevalence of $\sim 14 \%$, they are highly disabling and socioeconomically burdening, and function as precursors of other mental disorders such as depression or substance abuse disorders. Thus, preventive measures are urgently warranted to reduce the incidence of this disorder group and their sequential comorbidities.

The present talk provides an overview of existing preventive interventions aiming at reducing the incidence or severity of anxietyrelated phenotypes which are primarily based on cognitive-behavioral principles and - given that most anxiety disorders first manifest early in life - have so far mainly been directed at children/adolescents (e.g., 'FRIENDS', 'Cool Little Kids', 'REACH for RESILIENCE', 'Coping and Promoting Strength'), while only a few studies addressed adults at risk (e.g., 'Anxiety Sensitivity Amelioration Training', 'Anxiety Sensitivity Education and Reduction Training', 'Cognitive Anxiety Sensitivity Treatment [CAST]') (for review see Fisak et al. 2011; Moreno-Peral et al. 2017). Exemplarily, preliminary results of an own study showing the German version of CAST to be effective in reducing anxiety sensitivity, while at the same time indirectly decreasing separation anxiety, will be presented.

The integration of biological markers beyond psychological risk states might open up new avenues in the targeted prevention of anxiety by identifying high-risk individuals and thus informing indicated interventions and potentially also by inspiring innovative interventions directly targeting altered biological mechanisms underlying the onset of anxiety disorders such as bio- and fMRI(neuro)feedback-based methods or pharmacological approaches (cf. Domschke 2021a; Domschke 2021b).

References

Domschke, K., 2021. Prevention in psychiatry: a role for epigenetics? World Psychiatry 20, 227-228.

Domschke, K., 2021. Targeted prevention of anxiety disorders. Eur Neuropsychopharmacol 46, 49-51.

Fisak, B.J. Jr., Richard, D., Mann, A., 2011. The prevention of child and adolescent anxiety: a meta-analytic review. Prev Sci 12, 255-268.

Moreno-Peral, P., Conejo-Cerón, S., Rubio-Valera, M., Fernández, A., Navas-Campaña, D., Rodríguez-Morejón, A., Motrico, E., Rigabert, A., Luna, J.D., Martín-Pérez, C., Rodríguez-Bayón, A., BallestaRodríguez, M.I., Luciano, J.V., Bellón, J.Á., 2017. Effectiveness of psychological and/or educational interventions in the prevention of anxiety: a systematic review, meta-analysis, and meta-regression. JAMA Psychiatry 74, 1021-1029. 


\section{4}

PACAP-PAC1R system in females: contributions to fear learning and adaptations to stressors

\section{Prof. Raul Andero ${ }^{1}$ \\ ${ }^{I}$ Autonomous University of Barcelona, ICREA, Barcelona, Spain}

Posttraumatic stress disorder (PTSD) is more prevalent in women and has been associated with sex-specific mechanisms such as sex hormones and the PACAP-PAC1R (pituitary adenylate cyclaseactivating polypeptide and its type I receptor) system which confers risk in women but not men. Here, we show how the menstrual/estrous cycle at the moment of trauma does not predict PTSD-like symptoms in both women and female mice. However, chemogenetic manipulation of the medial amygdala projections to the dorsomedial part of the ventromedial hypothalamus during stress immobilization modulates the PACAP neurons preventing the impaired fear extinction phenotype. Our results highlight the role of a novel neural projection that may be relevant for PTSD prevention.

\section{5}

\section{Stress-induced changes in DNA-methylation: focus on anxiety disorders}

\section{Prof. Angelika Erhardt-Lehmann ${ }^{2}$ \\ ${ }^{1}$ Psychiatric Clinic University Würzburg, Würzburg, Germany, ${ }^{2}$ Max Planck Institute for Psychiatry, Germany}

Anxiety disorders (AD) are the most prevalent psychiatric conditions in the general population with an estimated lifetime prevalence up to $20 \%$. According to the existing evidence, genetic and environmental factors contribute to the etiology of AD. Approximately $40 \%$ of disease etiology is assigned to genetics, consisting of common and rare variations across the genome. Following this, environmental influences are etiologically highly relevant on shaping biological processes which lead to the risk for clinical symptomatology. Stress is one of the candidate environmental triggers associated with higher risk for $\mathrm{AD}$ in general. Environmental influences can induce longlasting alterations in neurobiological systems, such as endogenous stress system, e.g. mediated by epigenetic mechanisms. One of the epigenetic mechanisms increasingly studied in psychiatric research is DNA methylation, which occurs on cytosines through addition or removal of a methyl-group. In consequence, this process modulates gene expression by regulating the accessibility of transcription factors to their binding sites. There is first positive evidence of DNAmethylation changes in stress system related candidate genes, e.g. Corticotropin Releasing Hormone Receptor 1 (CRHR1) and Pituitary Adenylate Cyclase Activation Polypeptide (PACAP) genes, in various $\mathrm{AD}$ as disease category as well as in dimensional analysis including the environmental stress information. Further research with integration of detailed environmental life event information, such as timepoint and intensity/quality of stressors as well as positive environmental influences, are needed to better understand the DNAmethylation dynamics and their specificity for various $\mathrm{AD}$.

\section{6}

The endocannabinoid system in social anxiety

Mashal Ahmed ${ }^{1,3}$, Isabelle Boileau ${ }^{1,2,3,4,5}$, Bernard Le Foll ${ }^{1,2,3,4,5}$, Prof. Dr. Stefan Kloiber ${ }^{1,2,3,4,5}$

${ }^{1}$ Department of Psychiatry, University Of Toronto, Toronto, Canada, ${ }^{2}$ Campbell Family Mental Health Research Institute, Centre for Addiction and Mental Health, Toronto, Canada, ${ }^{3}$ Institute of Medical Science, University of Toronto, Toronto, Canada, ${ }^{4}$ Brain Health Imaging Centre, Centre for Addiction and Mental Health, Toronto, Canada, ${ }^{5}$ Department of Pharmacology and Toxicology, University of Toronto, Toronto, Canada

Social anxiety disorder (SAD) is among the most prevalent mental disorders worldwide and is characterized by early age of onset, chronic course, frequent psychiatric comorbidities and high levels of social dysfunction and disability. A large proportion of patients with SAD do not respond to currently available conventional treatments such as antidepressants and psychotherapy, which indicates the need for investigation of novel neurobiological mechanisms to identify targets for new treatments.

Emerging preclinical evidence suggests a potential role of the Endocannabinoid System (ECS) in the regulation of anxiety and social behavior, though only a few clinical studies have investigated the role of ECS signaling in SAD. In addition, cannabinoids are increasingly proposed as treatment and used by individuals with anxiety symptoms or anxiety disorders despite very limited evidence from clinical studies.

This presentation will include an overview of current knowledge on the potential role of the ECS in SAD, including discussion of prominent preclinical and clinical findings. Results from a comprehensive systematic literature review evaluating the current status of preclinical evidence on how pharmacological and genetic manipulations of components of the ECS such as cannabinoid receptors and enzymes degrading endocannabinoids, e.g. fatty acid amide hydrolase (FAAH), may affect social behaviour in different animal models will be presented. In addition, initial findings from a clinical neuroimaging study evaluating the ECS will be discussed. FAAH levels in the brain were measured in individuals with $\mathrm{SAD}$ using positron emission tomography (PET) imaging with the FAAH radioligand [C11]CURB. Preliminary findings indicate that high FAAH levels in the brain are associated with social anxiety and severity of SAD symptoms.

Current evidence indicates a potential role of the ECS in the regulation of social anxiety. Further preclinical and in particular clinical research is required to improve our understanding of function and effect of specific ECS components in anxiety and social behaviour. Findings of up-regulated brain FAAH activity as a potential pathophysiological mechanism in SAD could inform ECS- or specifically FAAH-targeted interventions.

77

\section{Selective mutism: The "unsafe world model"}

PD. Dr. Siebke Melfsen ${ }^{1}$, PD Thomas Jans ${ }^{2}$, Prof. Marcel Romanos ${ }^{2}$, Prof. Susanne Walitza ${ }^{1}$

${ }^{1}$ University of Zurich, Zurich, Switzerland, ${ }^{2}$ University Hospital of Wuerzburg, Wuerzburg, Germany

Selective mutism (SM) is still a challenging disorder. It is characterized by an absence of speech in selected situations in which children are expected to speak. However, the children do not show a physical disability to speak. The most common explanation for SM is 
an underlying anxiety disorder. However, there are several findings that cannot fully be explained by anxiety. In order to broaden the scope of other factors that might play a role in the aetiology of SM, a new model is proposed: the "unsafe world model". According to this model, SM is a stress reaction to situations erroneously experienced as 'unsafe'. It assumes a high sensitivity to unsafety, whereby the nervous system triggers dissociation or freeze mode at relatively low thresholds.

We examine whether there is a correlation between SM, sensoryprocessing sensitivity and dissociation. In a first study, we compared a sample of children and adolescents with SM to controls without SM. In a second study, we compared two groups of preschool children with and without SM. The groups were compared using different questionnaires. The results indicate that sensory-processing sensitivity was significantly higher in the SM-groups. Furthermore, dissociative symptoms were more common in SM groups than in controls. The results indicate that sensory-processing sensitivity and dissociation are relevant parameters of SM that may hold important implications for the treatment.

\section{8}

\section{An ecological momentary music intervention for the reduction of stress in the daily life of Turkish immigrant women}

\author{
Stefanie Hirsch ${ }^{1}$, BSc MSc Anja Feneberg ${ }^{1}$, Professor, PhD Urs \\ Nater ${ }^{1}$, PhD Ricarda Mewes ${ }^{2}$ \\ ${ }^{1}$ Department of Clinical and Health Psychology, Faculty \\ of Psychology, University Of Vienna, Vienna, Austria, ${ }^{2}$ Outpatient \\ Unit for Research, Teaching and Practice, Faculty of Psychology, \\ University of Vienna, Vienna, Austria
}

Research context: The experience of unfair treatment because of one's ethnicity, i.e., so-called perceived ethnic discrimination, is not a marginal problem. While both men and women are affected by this phenomenon, women may be additionally confronted with intersectional stigmata, i.e., both ethnic-related and gender-related, and thus constitute a particularly vulnerable group. Repeated exposure to such stressors, i.e., chronic discrimination, might lead to dysregulations in psychobiological stress systems. Considering the resulting negative health consequences, it is of great importance to provide adequate interventions. With regard to the occurrence of discrimination in everyday life and the acute psychobiological reactions to it, interventions need to be applied as early as possible. Recognizing this necessity, the pilot study investigates the feasibility and the effectiveness of a music-based ecological momentary intervention to reduce stress as a low-threshold strategy in the everyday lives of affected individuals.

Methods: 20 Turkish immigrant women who experience chronic ethnic discrimination take part. The whole study consists of a baseline period (week 1), an intervention period (weeks 2, 3, 4), and a post period (week 5). Using a smartphone-based app, participants are signaled 3 times a day (i.e., time-contingent data entries) to report their momentary stress levels and perceived discrimination. Additionally, participants initiate event-based data entries after the occurrence of every discriminatory or other stressful event. Subsequently, two additional data entries are triggered automatically after 20 and $35 \mathrm{~min}$. Every data entry is accompanied by the collection of a saliva sample for the analysis of biological stress markers (cortisol, alpha-amylase). The described sampling protocol is maintained during the whole study period. Additionally, during the intervention period, participants are randomly assigned (50:50) to either the intervention condition (i.e., listening to self-selected, personally relaxing music for $20 \mathrm{~min}$ ) or the control condition (i.e., no musiclistening for $20 \mathrm{~min}$ ) after every self-initiated event-based data entry. Using this intraindividual-randomized design, the immediate effects of music-listening on psychobiological stress parameters can be determined comparing psychological and biological data across different measurement time points (before vs. after music-listening, intervention-event vs. control-event). The intermediate effect of the whole intervention on psychobiological stress parameters can be examined comparing psychological and biological data from baseline vs. post period. Data will be analyzed using multilevel modeling. To investigate the feasibility of the intervention, qualitative data from semi-structured interviews on user-experience, burden, and satisfaction with the intervention will be gathered. Therefore, participants are invited into the laboratory after completion of the whole study period. Results: By the time of the congress, information on the current progress of the project will be available.

Implications: This study aims to promote health by empowering immigrant women to cope with discriminatory and stressful events in their everyday life. Additionally, significant input will be provided concerning the feasibility and effectiveness of a music-based ecological momentary intervention to reduce stress due to perceived ethnic discrimination in everyday life.

Keywords: Alpha-amylase, cortisol, ecological momentary intervention, ethnic discrimination, immigration, music, stress reduction

\section{9}

\section{Kynurenines, anxiety, and dementia}

\section{Professor László Vécsei, Dr. Masaru Tanaka}

${ }^{1}$ MTA-SZTE, Neuroscience Research Group, Szeged, Hungary, ${ }^{2}$ Department of Neurology, Interdisciplinary Excellence Centre, Albert Szent-Györgyi Medical School, University of Szeged, Szeged, Hungary

The tryptophan (TRP)-kynurenine (KYN) metabolic pathway is the major route of TRP metabolism, which produces a wide variety of bioactive molecules, including oxidants, antioxidants, neurotoxins, neuroprotectants, and immune modifiers. The activation of the pathway is observed in patients with cancer or immunologic, neurodegenerative, or psychiatric diseases. Anxiety is one of the most common manifestation of the spectrum of symptoms in comorbidity. The alteration of the TRP metabolism was reported in generalized anxiety disorder. Dementia is major neurocognitive disorders which dominate in patients who suffer from Alzheimer's disease and after a stroke. The disturbance of the TRP-KYN pathway was also reported in dementia. The TRP-KYN metabolism is upregulated by bacterial lipopolysaccharides, stress hormone cortisol, proinflammatory cytokines, and KYN metabolites such as L-KYN, 3-hydroxykynurenine, and nicotinamide adenine dinucleotide. Quinolinic acid is known as an excitotoxic ligand for the N-methyl-D-aspartate receptors. Intriguingly, the TRP-KYN pathway synthesizes various protective biomolecules such as kynurenic acid, anthranilic acid, picolinic acid, and cinnabarinic acid. However, none of them have been characterized well. The beneficial use of protective KYN derivatives is under extensive study. The blood-brain-barrier permeable halogenated kynurenic acid analogues have been designed. L-4-Chlorokynurnine, AV-101 entered phase 2 clinical trials, but its efficacy against major depressive disorder remains inconclusive. Depression, anxiety, and cognitive impairment are common symptoms linked to stressful conditions such as in inflammatory response, headache, dementia, and post-stroke. More novel kynurenic acid analogues have been synthesized to study their biological properties in search of new lead compounds for inflammation, migraine, and 
stroke. This presentation reports the most recent advances in KYN research, including the design of $\mathrm{KYN}$ analogues and their possible application for the treatment of stress, anxiety, and dementia.

Funding: This research was funded by GINOP 2.3.2-15-201600034, GINOP 2.3.2-15-2016-00048, TUDFO/47138-1/2019-ITM, TKP2020 Thematic Excellence Programme 2020, and OTKA 138125.

\section{0}

\section{Heart rate variability biofeedback for stress management supported by virtual reality and wearable technology}

MSc Jasmine Kerr ${ }^{1}$, Raphael Weibel ${ }^{1}$, Mara Naegelin ${ }^{1}$, Victor Schinazi $^{2}$, Andrea Ferrario ${ }^{1}$, Urs Nater ${ }^{3}$, Roberto La Marca ${ }^{4}$, Christoph Hoelscher ${ }^{1}$, Florian von Wangenheim ${ }^{1}$

${ }^{1}$ ETH Zürich, Zurich, Switzerland, ${ }^{2}$ Bond University, Gold Coast, Australia, ${ }^{3}$ University of Vienna, Vienna, Austria, ${ }^{4}$ Clinica Holistica Engiadina, Susch, Switzerland

Background: Heart rate variability biofeedback (HRV-BF) can be used for stress management as it regulates the activity of the autonomic nervous system. This is achieved by increasing HRV through slow and deep breathing. Virtual reality and head-mounted displays (HMD) for HRV-BF have gained attention, since they are believed to be associated with higher immersion, motivation and attentional focus than screen solutions. This study aims to test the feasibility of a selfdeveloped system and to compare the effects of HRV-BF and paced breathing only (PB) in combination with HMDs and screens.

Methods: One hundred and seven healthy students (48 female) were randomly assigned to one of four conditions: HRV-BF on a screen, HRV-BF via HMD, PB on a screen, or PB via HMD. Following psychoeducation, all participants practiced paced breathing for a duration of $2 \times 10$ min guided by a virtual pacer. The BF conditions were paced to breathe at their individual resonance frequency and received feedback based on HRV changes. Increases in HRV were computed every $10 \mathrm{~s}$ with the coherence ratio $(\mathrm{CR}=$ peak power/total spectral power between 0.04 and $0.26 \mathrm{~Hz}$ ). A CR $\geq 1$ increased the score by one unit and led to discrete changes in the virtual environment, whereas a $\mathrm{CR}<1$ had no influence. The breathing pace of $\mathrm{PB}$ conditions was fixed to 6 breaths per minute. We continuously measured cardiac activity with a chest belt and asked participants to fill out psychological state questionnaires pre, during and post intervention. Following the intervention, participants also rated their user experience (UX).

Results: Both HRV-BF (compared to PB) and HMD (compared to screen) led to the highest increases of the CR during the intervention (both $\mathrm{p}<.05$ ). Similarly, the low-frequency component of the HRV (LF) was significantly higher overall for HRV-BF compared to PB $(p=0.02)$. Nevertheless, all configurations were able to significantly increase the standard deviation of the NN (R-R) intervals (SDNN), $\mathrm{LF}$, and the CR, as well as decrease mean heart rate over time (all $\mathrm{p}<.001$ ). On a psychological level, all configurations led to significant yet indistinguishable changes. Specifically, increases in relaxation, calmness, good mood and wakefulness (all $\mathrm{p}<.001$ ), and decreases in perceived stress $(p<.001)$ and exhaustion $(p=.01)$ were observed over time. As for UX measures, participants were able to immerse themselves and get involved in either configuration, perceived that the required efforts were in line with their abilities, and felt competent and autonomous with regards to all four stress management interventions (all $\mathrm{p}<.05$ ).

Discussion: While this study showed that all technique and technology combinations are suitable for stress management purposes, practicing HRV-BF, instead of PB only, seems to be more promising with regard to increases in the $\mathrm{CR}$ and LF. In addition, opting for an HMD might even amplify the positive effects on the CR further. Future studies should consider investigating the effects of HRV-BF delivered via an HMD on psychophysiology and UX measures in a longitudinal setting.

81

\section{How do young people deal with a pandemic? Coping strategies used by Polish students during the COVID-19 lockdown}

Alicja Forma ${ }^{1}$, MD Elżbieta Sitarz ${ }^{2}$, Kaja Hanna Karakuła ${ }^{1}$, MD, PhD Jacek Baj ${ }^{3}$, MD, PhD Dariusz Juchnowicz ${ }^{4}, \mathrm{MD}$, PhD Jacek Bogucki $^{5}$, Professor, MD, PhD Hanna Karakuła-Juchnowicz ${ }^{2}$

${ }^{1}$ Psychiatry Student Research Group I Department of Psychiatry, Psychotherapy and Early Intervention, Medical University of Lublin, 20-439 Lublin, Poland, Lublin, Poland, ${ }^{2}$ Chair and I Department of Psychiatry, Psychotherapy, and Early Intervention, Medical University of Lublin, 20-439 Lublin, Poland, Lublin, Poland, ${ }^{3}$ Chair and Department of Anatomy, Medical University of Lublin, 20-090 Lublin, Poland, Lublin, Poland, ${ }^{4}$ Department of Psychiatric Nursing, Medical University of Lublin, 20-093 Lublin, Poland, Lublin, Poland, ${ }^{5}$ Chair and Department of Organic Chemistry, Faculty of Pharmacy Medical University of Lublin, 20-093 Lublin, Poland, Lublin, Poland

Background: COVID-19 affects lifestyle, well-being, and mental health for over a year now. The major fears of contracting the infection are mainly related to psychological, behavioral, and cognitive impairments. Students are reported to be the most vulnerable group characterized by one of the highest depression, anxiety, and stress levels. Stress coping strategies are mainly dependent on personality traits and are in a close relationship with the severity of mental and emotional distress. Furthermore, choices regarding coping strategies may imply the development as well as the severity of emotional disorders.

Purpose: The aim of the study is to present different coping strategies used by Polish students during the first wave of the COVID-19 pandemic and the relationship between them and the severity of the emotional distress. We also aimed to indicate which were the most effective, lowering the intensity of the anxiety, depression, and stress symptoms.

Methods: We conducted an online cross-sectional survey that was distributed amongst Polish students $(n=2172)$ from 87 universities on 20th-26th April 2020. We used Polish adaptations of two scalesthe Depression, Anxiety, and Stress Scale-21 (DASS-21) and the Carver Brief COPE Inventory to assess mental health and coping strategies respectively.

Results: We found that students more frequently choose 'approach' coping strategies $(\mathrm{M}=29.60 \pm 6.89)$ compared to 'avoidant' coping strategies $(\mathrm{M}=22.82 \pm 5.78)$. Using strategies as emotional support $(\mathrm{p}<0.001)$, positive reframing $(\mathrm{p}<0.001)$, acceptance $(\mathrm{p}<0.001)$, religion $(p=0.012)$, and humor $(p<0.001)$, was negatively correlated with the overall level of emotional distress and reduced its intensity. Contrarily, behavioral disengagement $(\mathrm{p}<0.001)$, venting $(p<0.001)$, self-blame $(p<0.001)$, and planning $(p=0.007)$ strengthen its severity. The results showed that the intensification of distress in women causes a turn to religion ( $p=0.001)$, while men use substances $(p<0.001)$ and a sense of humor $(p<0.001)$.

In relation to the field of study the greatest differences were found between medical and science students regarding DASS total score $(\mathrm{z}=3.312, \quad \mathrm{p}=0.009)$ and depression $(\mathrm{z}=3.25, \quad \mathrm{p}=0.012)$. Regardless of the students' health status, the respondents more often 
chose acceptance. However, those who suffered from mental disorders used avoidant coping strategies-self-distraction $(\mathrm{M}=1.78 \pm 0.76)$ and venting $(\mathrm{M}=1.67 \pm 0.85)$ —in addition to acceptance which was also most frequently chosen.

Conclusions: Medical students coped best with emotional distress, which is very encouraging for their future profession related to the need to work in a pandemic. Prior use of the help of a psychiatrist or psychologist, a sense of loneliness, and the usage of avoidant coping strategies were associated with the highest level of DASS total score. Identification of factors contributing to increased emotional distress is an essential factor in planning interventions preventing emotional disorders in the group of students.

Keywords: mental health; COVID-19 pandemic; coping strategies; depression; anxiety; stress;

The authors declare no conflict of interest.

82

Facial emotion recognitions are associated with suicide ideation in drug-naïve patients with major depressive disorder

\section{Yuting Wang ${ }^{1}$, Nanage Guobule ${ }^{1}$, Meijuan $\mathrm{Li}^{1}$, Dr. Jie $\mathbf{L i}^{1}$ \\ ${ }^{I}$ Tianjin Anding Hospital, Mental Health Center Of Tianjin Medical University, Tianjin, China}

Background: The ability of facial emotion recognition is often impaired in Major depressive disorder (MDD). The impairment of recognizing facial emotions may contribute to the disruption of interpersonal interaction. Perceived social isolation and social rejection are risk factors for suicide. The purpose of the present study is to investigate the ability of facial emotion recognition in drug-naïve MDD patients with first-episode with suicidal ideation.

Methods: Ninety-eight drug-naïve outpatients with first-episode of depression were recruited, including 53 patients with suicidal ideation (SI) and 45 patients without suicidal ideation (NSI). The Beck Scale for Suicide Ideation-Chinese Version was adopted to evaluate the suicidal ideation and suicidal risk in the latest week. Depressive symptoms were assessed with the Hamilton Depression Rating Scale17 (HDRS-17). Facial Emotion Recognition Test and the repeatable battery for the assessment of neuropsychological status (RBANS) were administered to assess the ability of emotion recognition and cognitive function. The Binary Logistic Regression was employed to investigate the relationship between facial emotion recognition and suicidal ideation and Forward stepwise linear regression was used to examine the suicidal risk in patients with suicidal ideation. $\mathrm{P}$ value $<0.05$ was considered statistically significant based on the two-tailed design-based test, with Bonferroni corrections for multiple comparisons.

Results: Our finding indicated that recognizing fear emotions was more accurate in the SI than the NSI group $(\mathrm{OR}=1.142 ; 95 \% \mathrm{CI}=$ $1.020,1.280 ; \mathrm{p}=0.022)$. Patients with high suicidal risk may have more difficulties recognizing the neutral facial expressions $(\mathrm{B}=-$ $0.310,95 \% \mathrm{CI}=-5.031,-0.845, \mathrm{p}=0.007)$.

Conclusions: Our results indicated that compared with patients without suicidal ideation, first-episode drug-naïve MDD patients with suicidal ideation recognized fear emotions more accurately. In addition, the increased suicide risk is associated with the impaired recognition of neutral emotions. The present study supports that changes of the abilities to recognize facial expressions of emotions may represent a risk factor for suicide. Given the effects of social cognition in suicide, facial emotion processing should be included in comprehensive, multifactorial models in future studies to identify suicidal ideations and to prevent suicidal behaviors in MDD patients.
Acknowledgements: This study was supported by Tianjin Key Project for Chronic Disease Prevention (No. 17ZXMFSY00070) and Tianjin Key Discipline Project for Psychiatry.

\section{3}

\section{COMT and CREB1 polymorphisms are associated with treatment-resistant major depressive disorder in Han Chinese}

Yuting Wang ${ }^{1}$, Dr. Shen $\mathrm{Li}^{2}$, Yanyan $\mathrm{Ma}^{1}$, Yuying Qiu ${ }^{1}$, Shuhua $\mathrm{Li}^{1}$, Nanage Guobule ${ }^{1}$, Haiyan Cao ${ }^{1}$, Dr. Jie $\mathbf{L i}^{1}$

${ }^{I}$ Tianjin Anding Hospital, Mental Health Center Of Tianjin Medical University, Tianjin, China, ${ }^{2}$ Tianjin Medical University, Tianjin, China

Background: Genetic factors play a crucial role for the pathophysiology of treatment-resistant depression (TRD). Three genome-wide association studies (GWAS) of TRD found that the genes related to cytoskeleton regulation and neural plasticity may be associated with TRD. It has been reported that Catechol-O-methyltransferase (COMT) and cyclic amp-response element-binding protein (CREB) are related to antidepressant response. To our knowledge, no study has investigated the role of CREB 1 and COMT genes in TRD in a Chinese population. The aims of the study are: (1) to investigate the relationship between genes COMT and CREB1 SNPs and TRD; (2) to explore the association between the SNPs of the genes COMT and CREB1 and the severity of symptoms of TRD; (3) to examine the interaction effects of the genes COMT and CREB1 as they related to the risk for TRD.

Methods: A total of 181 MDD patients (male/female: 63/118, average age: $44.22 \pm 11.44$ years, average duration: $25.20 \pm 32.00$ months) were recruited from the outpatient clinic of Tianjin Anding Hospital. Patients met the DSM-IV diagnostic criteria of MDD, confirmed by two experienced psychiatrists through the Structured Clinical Interview for DSM-IV (SCID). Among them, 81 patients met treatment-resistant depression, which is not responding to at least two different classes of antidepressants of adequate dose for at least 6 weeks. A total of 80 gender- and age-matched healthy controls (male/female: $25 / 55$, age: $40.39 \pm 11.589$ years) were also recruited from the the local community of Tianjin. Depressive symptoms were assessed with the Hamilton Depression Rating Scale17 (HDRS). Genotyping was performed using Mass Spectrometry. Genetic analyses were conducted by PLINK Software.

Results: The study showed that the distribution of COMT SNP rs4818 allele and genotypes were significantly different between TRD and controls $(\mathrm{P}=0.003)$, but no significant differences in SNPs of COMT and CREB1 genes between the non-TRD and the control groups. Statistical differences in allele frequencies were observed between TRD and non-TRD groups, including rs11904814 $(\mathrm{P}=0.001)$ and $\operatorname{rs6740584}(\mathrm{P}=0.031)$ in $\mathrm{CREB} 1$ gene, as well as rs4680 $(\mathrm{P}=0.036)$ and $\mathrm{rs} 4818(\mathrm{P}=0.016)$ in COMT gene. There were differences in the distribution of HDRS total scores among different phenotypes of CREB1 rs11904814, CREB1 rs6740584, COMT rs4680 and rs4818. Gene-gene interaction effect of COMTCREB1 (rs4680 $\times$ rs6740584) revealed significant epistasis in TRD $(\mathrm{P}<0.001)$.

Conclusions: The SNPs of CREB 1 and COMT increased the susceptibility of TRD and influenced the severity of depressive symptoms of MDD patients in a Chinese Han population. Our data support the idea that COMT and CREB1 genes have a genetic interaction that may increase the risk for developing TRD. Our data provides preliminary evidence to support a possible interaction effect 
between COMT and CREB1 genes in patients with TRD and points toward a potential mechanism of epistasis.

Acknowledgements: This study was supported by Tianjin Key Project for Chronic Disease Prevention (No. 17ZXMFSY00070) and Tianjin Key Discipline Project for Psychiatry.

\section{4 \\ The effect of berberine as an add-on treatment for weight control in patients with schizophrenia}

\author{
Yuying Qiu ${ }^{1}$, Meijuan $\mathrm{Li}^{1}$, Yonghui Zhang ${ }^{1}$, Ying Liu ${ }^{1}$, Yongping \\ Zhao $^{1}$, Jing Zhang ${ }^{1}$, Qiong Jia ${ }^{1}$, Dr. Jie $\mathbf{L i}^{1}$ \\ ${ }^{1}$ Tianjin Anding Hospital, Mental Health Center Of Tianjin Medical \\ University, Tianjin, China
}

Background: Berberine is an isoquinoline alkaloid extracted from Chinese herbs including coptis, cortex phellodendron, and rhizoma coptidis, which has been used for the treatment of gastrointestinal infections for thousands of years. In recent decades, berberine has been proved to present various pharmacological actions, including anti-diabetic, anti-dyslipidemia, against cancer, alleviating cardiovascular diseases and reducing the proinflammatory status. Berberine could improve antipsychotic-induced weight gain in obese cells lines and animal models. Furthermore, the effect of berberine on weight loss has also been replicated in the non-psychiatric population. However, there is no clinical trial for the application of berberine in patients with schizophrenia. In this study, the effects of berberine on body weight and body mass index (BMI) were examined, and the role of serum leptin and adiponectin were explored.

Methods: This was a randomized, double-blind, placebo-controlled trial. Patients with schizophrenia were recruited, who had taken a stable dose of antipsychotics for more than 1 month. Berberine (900 mg/day) or placebo were given as an add-on treatment for 8 weeks. Anthropometric parameters were measured at baseline, week 4 , and week 8 . The levels of peripheral blood leptin and adiponectin were detected at each time point.

Results: Sixty-five patients from Tianjin Anding Hospital were recruited, 49 of which completed the 8-week trial. Forty-five patients completed the 8 -week treatment, 27 patients $(77.1 \%)$ in the berberine group, and 22 patients $(73.3 \%)$ in the placebo group. At the end of 8th week, patients in the berberine group lost $1.10 \mathrm{~kg}$ weight, while $1.45 \mathrm{~kg}$ weight gain in the placebo group. There was a significant difference on body weight $(\mathrm{F}=10.493, \mathrm{p}=0.001)$, $\mathrm{BMI}(\mathrm{F}=9.344$, $\mathrm{P}=0.002)$ and serum leptin $(\mathrm{F}=6.265, \mathrm{P}=0.003)$. Further, the partial correlation analysis showed significant positive relationships between the changes of leptin and the decrease of body weight and body mass index. There was no significant difference in adverse events between the two groups $(\mathrm{P}>0.05)$. No patient experienced a serious adverse event during the study.

Discussion: Our results are consistent with previous studies with cell lines or animal models, that berberine inhibited adipogenesis and suppressed white adipose tissue accumulation. Decreased leptin levels after 8 weeks in berberine group suggested an improvement of leptin resistance by berberine, which is consistent with studies in rodents. Current doses of berberine have been used as an anti-inflammatory for decades without any toxicity, but whether it can be used in the long term remains to be confirmed.

Conclusion: This study suggests that berberine is a potential weight loss and weight maintenance drug for patients with schizophrenia. The effect of berberine on weight gain may be related to the regulation of leptin, but not adiponectin.
Acknowledgements: This study was supported by Tianjin Key Project for Chronic Disease Prevention (No. 17ZXMFSY00070) and Tianjin Key Discipline Project for Psychiatry.

\section{5}

\section{Prediction of anxiety and depression from polygenic scores in Swedish twins}

Mr Fara Tabrizi ${ }^{1}$, Victor Rahimzadeh William-Olsson ${ }^{1}$, Dr Jörgen Rosén ${ }^{1}$, Hampus Grönvall ${ }^{1}$, Prof. Erik Arner ${ }^{1}$, Prof. Patrik Magnusson $^{2}$, Dr Camilla Palm ${ }^{2}$, Prof. Henrik Larsson ${ }^{2}$, Dr Alexander Viktorin $^{2}$, Jens Bernhardsson ${ }^{1}$, Dr Billy Jansson ${ }^{1}$, Prof. Fredrik Åhs ${ }^{1}$

${ }^{1}$ Mittuniversitetet, institutionen för Psykologi och Socialt arbete, Östersund, Sweden, ${ }^{2}$ Karolinska Institutet, Stockholm, Sweden

Recent genome-wide association studies (GWAS) have identified several common variants associated with depression (Howard etal. 2019; Levey et al. 2021) and anxiety disorders (Levey et al. 2020; Meier et al. 2019; Purves et al. 2020), and these findings have been harnessed to develop polygenic scores (PGS) in order to provide an overall measure of individuals' genetic liability to develop disease (Torkamani et al. 2018). Research on the utility of PGSs as predictors of risk for disease is gaining traction, with studies on somatic illness showing that disease risk increases sharply in the right tail of the PGS distribution (Khera et al. 2018). Thus, PGS stratification could be of clinical relevance if it provides an opportunity to target those in need of preventive interventions with increased precision. The current potential of PGS stratification for depression and anxiety disorders remains an open question. In the current study, we applied 36 predefined PGSs from the polygenic index repository (Becker et al. 2021) on a target sample of 11,210 genotyped twins. Cases were defined as those with prescribed medication, where the prescription explicitly stated that a drug was ordinated for indication of depression or anxiety, respectively. Drugs included antidepressants (SSRI and SNRI), Benzodiazepines, Antihistamines, Buspirone, and Betablockers. As there were no indications of significant nesting within monozygotic (MZ) or dizygotic (DZ) twins, we used binary logistic fixed-effects models and change in pseudo-R2 above a baseline model with sex, age at survey, the first 20 ancestral principal components (PCs), and cohort to estimate the predictive performance of PGSs. In addition to the predictive power of individual PGSs, we estimated the conditional effect of all PGSs on our outcome measures, in order to examine whether each PGS had a unique contribution to the estimated risk of having anxiety- or depression medication. Finally, PGSs were divided at the 90th percentile to examine the possible increase in risk of depression- or anxiety medication in the right tail of the PGS continuum. The best predictive performance was estimated to $\Delta \mathrm{R} 2=0.029$ and 0.033 for depression- and anxiety disorders, respectively, when all PGSs were included in the same model. PGS for depression was the single best predictor of disease, where individuals in the top $10 \%$ of the PGS distribution had greater odds of having depression $(\mathrm{OR}=1.62 ; 95 \% \mathrm{CI}=1.37,1.91)$ or anxiety disorders $(\mathrm{OR}=1.66 ; 95 \% \mathrm{CI}=1.43,1.94)$ compared to the bottom $90 \%$ of the continuum. In conclusion, while PGS stratification does provide additional information regarding the identification of risk groups for depression or anxiety disorders, they are of limited clinical utility in their current form. 


\section{6}

\section{Sleep EEG as biomarker of emotional regulation and depression across the lifespan}

Prof. Dr. Martin Hatzinger ${ }^{1}$, Serge Brand, Edith HolsboerTrachsler, Ulrich M. Hemmeter, Diana Spieker, Axel Steiger, Marcel Zeising, Thorsten Mikoteit

${ }^{1}$ Psychiatric Services Solothurn and University of Basel, Basel, Switzerland

Sleep regulation is associated with psychiatric disorders such as depression. These sleep disturbances are related to hypothalamic-pituitary-adrenocortical (HPA) system functioning. In order to characterize sleep as biomarker, a first longitudinal study over several years in depressed patients was carried out by using polysomnography and HPA axis measurements. Results showed that sleep EEG variables were predictive for long-term outcome of the disorder. In a further step, sleep EEG markers reflected by the prefrontal theta cordance in REM sleep were investigated in the guidance of acute antidepressant treatment. First results confirm that these markers may be a reliable tool to tailor antidepressant therapy and, thus, improve acute treatment outcome significantly. However, most of these neurobiological data available so far are from studies after the disorder's onset. Therefore, a project was started in children in order to investigate sleep regulation, HPA axis function, and psychological/ behavioral variables. After the baseline examination at the age of 5 years participants were re-evaluated at the age of 6,9 , and 14 years as well. It could be demonstrated that some neurobiological markers were predictive for psychological/behavioral abnormalities later in life. In conclusion, sleep investigation by the use of objective sleep EEG markers is a promising approach in the prediction and in the guidance of treatment of affective disorders.

\section{7}

\section{Hippocampal activation predicts lower future PTSD symptoms}

\section{Dr. Tanja Jovanovic ${ }^{1}$, Sanne van Rooij \\ ${ }^{1}$ Wayne State University, Detroit, United States}

Understanding the neurobiological mechanisms that predict severity of posttraumatic stress disorder (PTSD) symptoms in trauma survivors is important for developing optimal early interventions. Impaired inhibition is central to PTSD symptomatology, but its role in predicting PTSD is unknown. Prior neuroimaging studies have identified lower ventromedial prefrontal cortex (vmPFC) and hippocampal activation during inhibition paradigms as contributors to the development of PTSD. Further, studies have identified clear sex differences in risk for PTSD, but most imaging studies are underpowered to test for interactions with sex. We examined whether brain function during response inhibition early after a civilian trauma predicted future PTSD symptoms in two studies that recruited patients from the Emergency Department (ED) within $24 \mathrm{~h}$ of trauma exposure. The first study was conducted at a single site $(\mathrm{N}=58)$, while the second study was part of the multi-site AURORA study $(\mathrm{N}=261)$. PTSD symptoms were assessed in the ED and 1, 3 and 6-months posttrauma. A Go/NoGo procedure in a $3 \mathrm{~T}$ MRI scanner was used to measure neural correlates of response inhibition post-trauma. Regression was used to define the most optimal model to predict PTSD symptoms at 6-months after trauma controlling for demographic, clinical and imaging measures. Results of the first study included an original and replication dataset; both showed that less hippocampal activation was a significant predictor in the model predicting PTSD symptoms at 6-months $(\mathrm{F}=4.96, \mathrm{p}=0.01$ and $\mathrm{F}=5.74, \mathrm{p}=0.007$, respectively). This finding may contribute to early identification of at-risk individuals and reveals potential targets for intervention or symptom prevention in the aftermath of trauma. Linear regression analyses including sex in the multi-site study showed a significant model for the vmPFC $(\mathrm{F}=4.33, \mathrm{p}=.014)$, with lower vmPFC activation $(\mathrm{B}=-.123, \mathrm{p}=.046)$ predicting 6 -month PTSD symptoms. A significant model for the hippocampus $(\mathrm{F}=4.61$, $\mathrm{p}=.033)$ showed that hippocampal activation and sex interacted in predicting 6-month PTSD symptoms $(\mathrm{B}=-.132, \mathrm{p}=.033)$. Specifically, lower hippocampal activation in men was related to greater 6-month PTSD symptoms $(\mathrm{r}=-.234, \mathrm{p}=.025)$. Taken together, these studies show that lower hippocampus activation during a response inhibition task is predictive of higher PTSD symptoms after trauma. Further, lower hippocampal activation was specifically related to later PTSD symptoms in men, whereas vmPFC activation predicted PTSD symptoms in both men and women. These results suggest response inhibition as a potential mechanism for post-trauma psychopathology that could be a target for early interventions.

\section{8}

\section{Peripheral biomarkers of depression vulnerability during adolescence and their potential impact on brain functioning.}

\section{Prof. Dr. Annamaria Cattaneo ${ }^{1}$ \\ ${ }^{1}$ University of Milan/IRCCS Fatebenefratelli Brescia, Milan, Italy, ${ }^{2}$ IRCCS Fatebenefratelli Brescia, Brescia, Italy}

Childhood trauma and emotional dysregulation represent two risk factors for the development of depression later in life. Childhood trauma is the experience of trauma during the infancy whereas emotional dysregulation is the ability of an individual to modify the emotional state in order to adapt to goal-oriented behaviors (Beauchaine TP, 2015, J Clin Child Adolesc Psychol. 44:875-896). Childhood trauma has been already associated with alterations in the peripheral pro-inflammatory cytokines, such as TNF-alpha, C-reactive protein (CRP) and IL-6 (Baumeister et al., 2016, Mol Psychiatry, 21(5):642-9), suggesting their possible utility as biomarkers for an early identification of adolescents at high-risk for developing depression. Also transcriptomic analyses have been proposed as useful tool to identify peripheral biomarkers and pathways associated with depression development or with the risk for it.

A group of 182 adolescents aged between 17 and 22 years old were recruited in Italian high-school and were clinically assessed by inviting them to fill-in self-report questionnaires. Saliva samples were collected by chewing cotton swab (Salivette, Sarsted) for one minute. The levels of the pro-inflammatory cytokine CRP were measured by ELISA immuno-essay (Salimetrics, USA), formatted to minimize cross reactivity for related cytokines. Other groups of adolescents characterized for childhood trauma and depressive symptoms have been also asked to provide blood samples that have been then used to run transcriptomic analyses. The statistical analysis were performed by using SPSS statistical analysis software or with Partek Genomic Suite Software.

Out of the 182 adolescents (age $17.86 \pm 0.91$ ), $57,1 \%$ experienced at least one type of trauma during childhood and the most common are emotional neglect (37.2\%) and emotional abuse (32.8\%), followed by sexual abuse $(18 \%)$, physical neglect $(12 \%)$ and physical abuse $(9.8 \%)$. It has been also showed a positive correlation between emotional dysregulation and childhood trauma $(\mathrm{R}=.494, \mathrm{p}<0.01)$. The CRP concentration in saliva has been successfully measured in 
144 adolescents and we found the presence of lower CRP levels in adolescents with higher scores in the childhood trauma. However, no significant correlation has been found with emotional dysregulation related scores. Transcriptomic analyses revealed alterations in inflammatory biomarkers ad related pathways in blood samples of adolescents with depressive symptoms, with an effect which was driven by male adolescents.

The positive correlation between childhood trauma and emotional dysregulation related scores suggests that the subjects who experienced traumatic events in childhood are more likely to develop emotional dysregulation during adolescence. In addition, lower CRP levels in adolescents with a history of childhood trauma might represents a biomarker related to stress copying mechanism. However, further cytokines such as IL-1beta and IL- 6 should be measured to better clarify the role of inflammation in mediating the effect of childhood trauma on emotional dysregulation. Blood transcriptomics seemed to be more sensitive in the identification of biomarkers and related pathways mapping adolescents exposed to childhood trauma and with higher risk for depression development.

\section{9}

\section{Emotion regulation and the autonomic nervous system in adolescent psychopathology}

\author{
Julian Prof. Dr. Koenig ${ }^{1}$ \\ ${ }^{1}$ University of Cologne, Faculty of Medicine and University Hospital \\ Cologne, Department of Child and Adolescent Psychiatry, \\ Psychosomatics and Psychotherapy, Cologne, Germany
}

Adolescents with psychopathology are frequently characterized by deficits in stress and emotion regulation. Successful stress and emotion regulation involves regulatory processes by the autonomic nervous system (ANS). The integrative action of the ANS in stress and emotion regulation is rooted in shared neural circuitry, i.e. brain areas involved in the regulation of stress and emotion are also involved in regulating ANS function. The talk will outline the neurobiological foundation of neurovisceral integration; highlight recent studies in the field of child and adolescent psychiatry addressing ANS function in association with dysregulated stress and emotion regulation, and discuss clinical implications concerning the therapeutic potential to target ANS function in child and adolescent psychiatry. First, the model of neurovisceral integration in stress and emotion regulation is presented. Heart rate variability (HRV) - a marker of the integrated action of the central nervous system and the ANS is introduced. Supporting evidence from neuroimaging studies, addressing the association of HRV with brain structure and function, is reviewed with a specific focus on developmental aspects and trajectories. Second, cross-sectional and longitudinal studies addressing HRV in association with stress and emotion regulation as well as symptom severity in adolescents with psychopathology are reviewed. It is suggested that reduced HRV may be a risk-factor for the development of psychopathology and is capable to monitor treatment progress and outcome in these patients. Finally, clinical implications of the aforementioned findings are discussed. It is suggested that targeting ANS function, i.e., via transcutaneous vagus nerve stimulation or pharmacological interventions, may offer new treatment options in child and adolescent psychopathology.

\section{0}

Kynurenines, anxiety, and dementia

\author{
Masaru Tanaka ${ }^{1,2}$, Prof. Dr. Laszlo Vecsei ${ }^{1,2}$ \\ ${ }^{1}$ MTA-SZTE, Neuroscience Research Group, Szeged, Hungary, \\ ${ }^{2}$ Department of Neurology, Interdisciplinary Excellence Centre, \\ Albert Szent-Györgyi Medical School, University of Szeged, Szeged, \\ Hungary
}

The tryptophan (TRP)-kynurenine (KYN) metabolic pathway is the major route of TRP metabolism, which produces a wide variety of bioactive molecules, including oxidants, antioxidants, neurotoxins, neuroprotectants, and immune modifiers. The activation of the pathway is observed in patients with cancer or immunologic, neurodegenerative, or psychiatric diseases. Anxiety is one of the most common manifestation of the spectrum of symptoms in comorbidity. The alteration of the TRP metabolism was reported in generalized anxiety disorder. Dementia is major neurocognitive disorders which dominate in patients who suffer from Alzheimer's disease and after a stroke. The disturbance of the TRP-KYN pathway was also reported in dementia. The TRP-KYN metabolism is upregulated by bacterial lipopolysaccharides, stress hormone cortisol, proinflammatory cytokines, and KYN metabolites such as L-KYN, 3-hydroxykynurenine, and nicotinamide adenine dinucleotide. Quinolinic acid is known as an excitotoxic ligand for the $N$-methyl-D-aspartate receptors. Intriguingly, the TRP-KYN pathway synthesizes various protective biomolecules such as kynurenic acid, anthranilic acid, picolinic acid, and cinnabarinic acid. However, none of them have been characterized well. The beneficial use of protective KYN derivatives is under extensive study. The blood-brain-barrier permeable halogenated kynurenic acid analogues have been designed. L-4-Chlorokynurnine, AV-101 entered phase 2 clinical trials, but its efficacy against major depressive disorder remains inconclusive. Depression, anxiety, and cognitive impairment are common symptoms linked to stressful conditions such as in inflammatory response, headache, dementia, and post-stroke. More novel kynurenic acid analogues have been synthesized to study their biological properties in search of new lead compounds for inflammation, migraine, and stroke. This presentation reports the most recent advances in KYN research, including the design of KYN analogues and their possible application for the treatment of stress, anxiety, and dementia.

Funding: This research was funded by GINOP 2.3.2-15-201600034, GINOP 2.3.2-15-2016-00048, TUDFO/47138-1/2019-ITM, TKP2020 Thematic Excellence Programme 2020, and OTKA 138125 .

\section{1}

\section{Modelling ADHD: potentials studying $\mathbf{G} \times \mathbf{E}$ interactions and therapy response}

\author{
Prof. Dr. Edna Grünblatt ${ }^{1,2,3}$ \\ ${ }^{1}$ Department of Child and Adolescent Psychiatry and Psychotherapy, \\ Psychiatric University Hospital Zurich, University of Zurich, Zurich, \\ Switzerland, ${ }^{2}$ Neuroscience Center Zurich, University of Zurich \\ and the ETH Zurich, Zurich, Switzerland, ${ }^{3}$ Zurich Center \\ for Integrative Human Physiology, University of Zurich, Zurich, \\ Switzerland
}

Attention-deficit hyperactivity disorder (ADHD) is the most frequent mental disorder in children and adolescents, persisting into adulthood in more than $60 \%$ of the patients. It is hypothesized that the brain maturation delays are linked both to its high genetic 
(G) predisposition and possibly environmental (E) factors e.g. prenatal stress known to influence child brain development. Multiple evidence has shown that methylphenidate (MPH), the first line treatment for $\mathrm{ADHD}$, mitigates brain maturation delays (e.g. prefrontal cortex/PFC), improving patients outcome. However, the molecular mechanisms underlying the processes involved in the etiology of the disorder and its treatment still need to be elucidated. Modelling neurodevelopmental processes using patients' specific cells seems to be a promising tool. Namely, using induced pluripotent stem cells (iPSCs) reprogrammed from patients somatic cells, iPSCderived neural progenitors (NPCs) and PFC neurons may help advancing our knowledge in the involvement of $\mathrm{G} \times \mathrm{E}$ factors and treatment. Already, our preliminary results show that iPSCs and NPCs from ADHD children exhibits higher proliferation rates than healthy controls, which might cause an opposite effect in differentiation rates. Interestingly, previously, glucocorticoid receptor (GR) activation (e.g. via dexamethasone) in iPSC-generated cerebral organoids or forebrain neurons point to differentiation and maturations delays effects through stress response, while the proliferation of NPCs were increased. Therefore, it is conceivable that ADHD phenotype might be induced via a combination of $\mathrm{G} \times \mathrm{E}$ factors, while treatment reverse it. However, this still needs to be investigated to draw any conclusion. Nevertheless, modelling ADHD using iPSC is promising to reveal treatment effects in ADHD at the molecular level and broaden possibilities to develop possible preventive approaches and discover new therapeutic targets.

\section{2}

\section{Dysregulation of brain arousal: a central pathogenetic factor in depression, mania and ADHD}

\section{Prof. Dr. Ulrich Hegerl ${ }^{1}$ \\ ${ }^{1}$ Department of Psychiatry, Psychosomatics, And Psychotherapy, Goethe Universität Frankfurt, Frankfurt Am Main, Germany}

The human brain can take over different global functional states not only during sleep (sleep stages, e.g. slow wave sleep, REM sleep) but also during wakefulness (arousal levels). A variety of clinical and preclinical studies consistently indicate that in major depression an upregulated brain arousal and in ADHD as well as mania an unstable brain arousal regulation play a crucial pathogenetic role. The hyperactivity and sensation seeking observed in overtired children, ADHD and mania is interpreted as an autoregulatory attempt of the organism to stabilize brain arousal level by increasing external stimulation. Correspondingly the withdrawal and sensation avoidance in,ajor depression is interpreted as a reaction to a state of tonically high brain arousal (1). The EEG-based algorithm (Vigilance Algorithm Leipzig, VIGALL, free download http://www.uni-leipzig. de/ vigall/) allows to objectively assess the level as well as the regulation of brain arousal within a 15-min EEG recording under quiet rest. Support for this concept is provided by by a variety of EEG-studies on patients with major depression, bipolar disorder and ADHD as well as by the finding in a recent GEWA. In this GEWA it was found that the genetic variant most closely associated with upregulated brain arousal (assessed with VIGALL 2.1.) has also been found by others to be associated with depression (2). Recent studies indicate that an upregulated brain arousal at baseline in major depression predicts response to antidepressants (3). Unstudied up to now is the question whether an upregulated arousal also predicts antidepressant response to ketamine or esketamine.

1)Hegerl \& Hensch 2012, Neurosci Biobehav Rev.

2)Jawinski et al. 2018, Molecular Psychiatry.

3)Schmidt et al. 2017, Sci Rep.

\section{3}

\section{Guiding antidepressant treatment with therapeutic drug monitoring and genetic testing}

\section{Andreas Menke ${ }^{1}$ \\ ${ }^{I}$ Medical Park, Bernau Am Chiemsee, Germany}

Major depression is a common disorder that implicates a huge burden for patients and society. Currently used antidepressants are very effective, however, more than $50 \%$ of patients do not respond to the first prescribed antidepressant. Additionally, in more than $25 \%$ of the patients treated with antidepressants adverse effects occur. Currently, the selection of an antidepressant is not based on measurable data from behavioral or neurobiological systems. However, in the last decades, the understanding of the underlying biological mechanisms of antidepressant metabolism and response has grown and can be used for the development of precision medicine to deliver a patient-tailored individual treatment. Already applied methods are the therapeutic drug monitoring (TDM) and genetic tests that predict the metabolism of drugs, including antidepressants. TDM quantifies the plasma concentrations of drugs for dose optimization. Typical situations for TDM are uncertain drug adherence, non-response to therapeutic doses, pharmacokinetic drug-drug interactions or suboptimal tolerability. Especially for vulnerable patients groups, i.e. children, elderly patients or pregnant women, TDM improves the safety of drug administration. Pharmacogenetics describe how genetic variation affects drug metabolism and response. Robust evidence was found that genetic variants of CYP450 enzymes such as CYP2C19 or CYP2D6 may predict the metabolism of certain drugs. In fact, numerous expert panels, such as the Clinical Pharmacogenetics Implementation Consortium (CPIC) or the Pharmacogenomics Knowledgebase (PharmGKB) recommend the detection of these variants, for example by using commercially available tests. However, precision medicine is still at the very beginning, tests that include imaging, elaborate blood tests, or consider gene $\mathrm{x}$ environment interactions, childhood trauma or severe stressful life events or proximal factors such as lifestyle, nutrition and sport are still not employed. The objective of many ongoing studies is to deliver evidence for precision medicine approaches to match individual patients with the most effective treatment options thus increasing efficacy and minimizing adverse drug reactions.

\section{4}

\section{Psychopathology of Parkinson's disease}

\section{Prof. Dr. Thomas Brücke ${ }^{1}$ \\ ${ }^{1}$ FWFW Wilhelminenspital, Vienna, Austria}

Psychopathology of Parkinson's Disease

T. Brücke

Parkinson's disease (PD) is the second most common neurodegenerative disorder with a prevalence of $1-2 / 1000$. The diagnosis of PD is based on the motor symptoms bradykinesia, rigidity and resting tremor, which are a consequence of the degeneration of dopaminergic neurons in the substantia nigra.

In recent years the focus of interest has shifted to the nonmotor symptoms of PD, some of which such as hyposmia, REM sleep behaviour disorder, constipation and depression, can precede the onset of clinically manifest disease for many years.

In advanced stages of the disease nonmotor symptoms are often the leading cause for the reduction of quality of life. Many PD patients with motor fluctuations (on-off) experience depressive 
symptoms, anxiety and bradyphrenia during off periods. Neuropsychiatric symptoms such as depression, anxiety, cognitive decline, fatigue, apathy and therapy induced hallucinations can dominate the clinical picture in later stages and often are the main reason for institutionalisation.

The clinical picture, the underlying pathophysiology and possible therapies are discussed and an overview of recent developments is given.

The existence of these different nonmotor symptoms which often do not respond to dopaminergic treatment underlines the fact that PD is a multisystem disorder which affects different neurotransmitter systems in widespread regions of the nervous system.

\section{5}

\section{Virtual technologies in the treatment of anxiety}

\author{
Dr. phil. Dipl.-Psych. Julia Diemer ${ }^{1,2}$ \\ ${ }^{1}$ kbo-Inn-Salzach-klinikum gGmbH, Anxiety Research Group, Health \\ Services Research, Wasserburg am Inn, Germany, ${ }^{2}$ Department \\ of Psychology, LMU Munich, Munich, Germany
}

Virtual reality (VR) has been studied as a medium to conduct exposure exercises in the psychotherapy of anxiety disorders for over two decades. Numerous studies document the efficacy of VR exposure therapy, in particular for specific phobia (Morina et al. 2015), and the efficacy data for VR exposure in other anxiety- and stress-related disorders are also promising (Carl et al. 2019; Diemer \& Zwanzger 2019; Kothgassner et al. 2019; Opris et al. 2012). In recent years, the field has witnessed technological advances that have opened up a number of innovative applications for VR. Most notably, portable and low-cost VR solutions have been developed, and are increasingly being incorporated into digital self-management interventions. Innovative VR technology also creates new potential for the investigation of stress- and anxiety-related disorders, including opportunities for therapy process research.

This talk aims at presenting current developments in virtual reality exposure, with a focus on recent use of this technology in the context of digital self-help programs. An outline of VR exposure therapy is given, followed by an overview of VR innovations in recent years. Self-management interventions-with or without VR exercises-are part of current debates surrounding e-mental health solutions. In particular, digital interventions might improve access to care, while patient safety and data protection are important concerns. Chances and perspectives of VR in this context are outlined, and potential risks and research gaps are discussed.

Conflict of interest: The author declares that she has no conflict of interest regarding the content presented in this talk.

Literature

Carl E, Stein AT, Levihn-Coon A, Pogue JR, Rothbaum B, Emmelkamp P, Asmundson GJG, Carlbring P, Powers MB (2019) Virtual reality exposure therapy for anxiety and related disorders: A meta-analysis of randomized controlled trials. Journal of Anxiety Disorders 61, 27-36.

Diemer J, Zwanzger P (2019) Die Entwicklung virtueller Realität als Expositionsverfahren. Der Nervenarzt 90(7), 715-723.

Kothgassner OD, Goreis A, Kafka JX, Van Eickels RL, Plener PL, Felnhofer A (2019) Virtual reality exposure therapy for posttraumatic stress disorder (PTSD): a meta-analysis. European Journal of Psychotraumatology https://doi.org/10.1080/20008198.2019.1654782

10:1654782.

Morina N, Ijntema H, Meyerbröker K, Emmelkamp PMG (2015) Can virtual reality exposure therapy gains be generalized to real life?
A meta-analysis of studies applying behavioral assessments. Behaviour Resarch and Therapy 74, 18-24.

Opris D, Pintea S, Garcia-Palacios A, Botella C, Szamoskozi S, David D (2012) Virtual reality exposure therapy in anxiety disorders: A quantitative meta-analysis. Depression and Anxiety 29 (2), 85-93.

\section{6}

\section{New strategies in the psychopharmacology of anxiety}

\section{Prof. Dr. Nicolas Singewald ${ }^{1}$ \\ ${ }^{I}$ Dept Pharmacology, Inst Pharmazie and CMBI, Univ Innsbruck,} Innsbruck, Austria

The pharmacological treatments for anxiety disorders and PTSD are suboptimal in terms of efficacy/long-term therapeutic benefit and tolerability, highlighting the need for further development of improved medication. Until now most anti-anxiety drugs were originally licenced for depression and also at present there are only few new drugs developed specifically for anxiety in the medication pipeline.

In this talk I will present an overview of the drugs being studied in different phases of clinical trials for generalised anxiety disorder, social anxiety disorder, panic disorder, phobias or PTSD and will discuss some select drug examples in more detail. Strategies followed in current anxiety drug development include (i) refining and improving compounds interacting with existing anxiolytic drug targets, such as serotonin and GABA systems, as well as combination of such targets (ii) the development of compounds with novel mechanisms of anxiolytic action identified by using the growing knowledge concerning the relevant neurocircuitries and neurobiological mechanisms underlying pathological fear and anxiety. Such target systems evaluated in current clinical trials include glutamate, endocannabinoid, neurosteroid, neuropeptide (e.g. vasopressin, orexin) systems, as well as ion channels and (multimodal) targets derived from phytochemicals and psychedelics. Interesting potential drug targets evaluated at the preclinical stage include epigenetic mechanisms such as HDAC inhibition, the inflammatory system, the microbiom and others. Finally, I will discuss in more detail research of a promising option for improving therapy outcome: the pharmacological boosting of fear-inhibition or extinction learning in exposure-based behavioral therapy (Singewald et al Pharmacol \& Ther 2015, Sartori and Singewald, Pharmacol \& Ther 2019). Compounds such as D-cycloserine, MDMA, cannabinoids and L-DOPA have shown efficacy in enhancing fear extinction learning in animals and healthy humans. They are thus investigated in clinical trials as an augmentative strategy for speeding up and enhancing the long-term effectiveness of exposure-based psychotherapy, or to assist other psychotherapeutic approaches. This could, in the future, render the need of chronic anxiolytic drug treatment dispensable for many patients, which would represent a paradigm shift in the use of anxiety pharmacotherapeutics.

\section{7}

Social attention under stress. Is gaze behavior associated with the psychobiological response to acute stress?

Dr. Bernadette von Dawans ${ }^{1}$, Carolyn Vatheuer, Antonia Vehlen, Gregor Domes

${ }^{1}$ Biological And Clinical Psychology, University Of Trier, Trier, Germany 
Background: The Trier Social Stress Test (TSST) is a reliable tool for psychobiological stress induction. Because of its socioevaluative nature, it has been useful for investigating gaze behavior. It has been shown that healthy people avoid looking toward faces when under stress, a finding that corroborates studies demonstrating avoidance of eye contact in social anxiety disorder. Yet, little is known about the relationship between gaze behavior and the biological stress response. Methods: In a final sample of 74 healthy males, a virtual reality version of the Trier Social Stress Test (TSST-VR) with an integrated eye tracker was implemented to investigate gaze behavior during acute stress induction. Stress response measures were collected via saliva samples and subjective stress ratings. Additional questionnaires were administered for examining the influence of social anxiety traits. Results: The TSST-VR elicited a significant psychobiological stress response. Overall, higher gaze times on judges compared to surroundings were found in the speech task while this pattern was reversed in the arithmetic task. Critically, there was a significant negative association between gaze time on judges and cortisol output in cortisol responders.

Conclusions: In a non-clinical sample, avoidance of gaze is associated with a stronger cortisol response to acute stress. This study demonstrates the potential of eye tracking to disentangle the effects of acute stress on social interaction, warranting further investigation in clinical populations characterized by high levels of anxiety in social situations, such as social anxiety and autism spectrum disorder.

\section{8}

\section{Nocturnal heart rate variability in children with autism spectrum disorder}

Dr. Eva-Maria Kurz ${ }^{1}$, Dr. Gottfried Maria Barth ${ }^{1}$, Prof. Dr. Annette Conzelmann ${ }^{1}$, Dr. Katharina Zinke ${ }^{2}$, Prof. Dr. Jan Born ${ }^{2}$, Prof. Dr. Tobias J. Renner

${ }^{I}$ Department of Child and Adolescent Psychiatry, Psychosomatics and Psychotherapy, University Hospital of Psychiatry and Psychotherapy, Tübingen, Germany, ${ }^{2}$ Institute of Medical Psychology and Behavioral Neurobiology, University of Tübingen, Tübingen, Germany

During wakefulness, individuals with autism spectrum disorder (ASD) have often been reported to show a diminished heart rate variability (HRV) in both resting conditions and as reaction to different tasks. Very little is known about nocturnal HRV in individuals with ASD, with current studies even reporting contradictory findings. A group of 19 nine to 12 years old children with ASD and 20 agematched typically developed (TD) children slept one night with polysomnography and a two-lead electrocardiogram. In the evening before sleep and about one hour after waking up in the morning the children performed an emotional picture recognition task. Consistent with previous studies, children with ASD compared to TD children showed a diminished parasympathetic activity during the encoding of emotional pictures. However, such altered cardiac responses were not seen during sleep. During sleep stage 2, slow wave sleep and rapid eye movement sleep, children with ASD and TD children showed a comparable HR and parasympathetic activity, assessed through highfrequency power $(0.24-1.04 \mathrm{~Hz})$ and RMSSD. Similarly sleep architecture was comparable between the groups. Undisturbed sleep physiology might also be reflected by the influence of parasympathetic activity during sleep.

\section{9}

Emotion regulation and the endogenous opioid system in adolescents and young adults with NSSI

Lisa Störkel $^{1,4}$, Dr Alexander Karabatsiakis ${ }^{2,4}$, Dr. Johanna Hepp ${ }^{1}$, Prof. Dr. Iris-Tatjana Kolassa ${ }^{3}$, Prof. Dr. Christian Schmahl ${ }^{1}$, PD Dr. Inga Niedtfeld ${ }^{1}$

${ }^{1}$ Department of Psychosomatic Medicine, Central Institute of Mental Health, Medical Faculty Mannheim at Heidelberg University, Mannheim, Germany, ${ }^{2}$ Department of Psychology, University of Innsbruck, Innsburck, Austria, ${ }^{3}$ Department of Psychology, University of Ulm, Ulm, Germany, ${ }^{4}$ These authors contributed equally

Non-suicidal self-injury (NSSI) is a prevalent and impairing behavior that affects individuals with and without additional psychopathology. Herein, we focused on biological mechanisms that precede and result from NSSI. Since previous cross-sectional evidence suggests that $\beta$ endorphin might be involved in the psychopathology of NSSI, we assessed salivary $\beta$-endorphin in daily life. We recruited 51 female adults with NSSI disorder according to DSM-5, participating over 15 days in an ambulatory assessment design. We assessed salivary $\beta$ endorphin before and after engagement in NSSI, on a non-NSSI day, and during high urge for NSSI. We also assessed pain ratings, method, severity, and function of NSSI. We found that $\beta$-endorphin levels immediately before an NSSI act were significantly lower than post NSSI samples. We observed a positive association of $\beta$-endorphin levels and the severity of the self-inflicted injury, but no significant association between $\beta$-endorphin levels and experienced pain. Finally, we found no significant differences between $\beta$-endorphin during a high urge control condition and post NSSI levels. Our study suggests that the assessment of salivary $\beta$-endorphin in the context of NSSI in daily life is possible. Furthermore, we conclude that NSSI could be associated with temporary increase of $\beta$-endorphin, and this might reinforce NSSI engagement.

\section{0}

Brain characteristics associated with anxiety disorders:
an update from the ENIGMA-Anxiety Working Group

Dr. Nynke A. Groenewold ${ }^{1,2}$, Janna Marie Bas-Hoogendam ${ }^{3,4}$, Moji Aghajani $^{5,6}$, Kevin Hilbert ${ }^{7}$, Andre Zugman ${ }^{4}$, Miquel A. Fullana ${ }^{8}$, Anita Harrewijn ${ }^{4,9}$, Elise M. Cardinale ${ }^{4}$, Eline Roelofs ${ }^{10}$, Max A. Laansma $^{11}$, Laura S. van Velzen ${ }^{12}$, Sophia I. Thomopoulos ${ }^{13}$, Neda Jahanshad $^{13}$, Anderson M. Winkler ${ }^{4}$, Paul M. Thompson ${ }^{13}$, Dick J. Veltman $^{5}$, Ulrike Lueken ${ }^{7}$, Daniel S. Pine ${ }^{4}$, Dan J. Stein ${ }^{1,14}$, Nic J.A. van der Wee ${ }^{10}$, for the ENIGMA-Anxiety Working Group

${ }^{1}$ Department of Psychiatry \& Mental Health; Neuroscience Institute, University of Cape Town, Cape Town, South Africa, ${ }^{2}$ Department of Paediatrics and Child Health, Red Cross War Memorial Children's Hospital; South African Medical Research Council (SA-MRC) Unit on Child \& Adolescent Health, University of Cape Town, Cape Town, South Africa, ${ }^{3}$ Department of Psychiatry, Leiden University Medical Center; Department of Developmental and Educational Psychology, Institute of Psychology, Leiden University; Leiden Institute for Brain and Cognition, Leiden, The Netherlands, ${ }^{4}$ Emotion and Development Branch, National Institute of Mental Health, National Institutes of Health, Bethesda, United States of America, ${ }^{5}$ Department of Psychiatry, Amsterdam UMC, Location VUMC, Amsterdam Neuroscience, Amsterdam, The Netherlands, ${ }^{6}$ Institute of Education \& Child Studies, Section Forensic Family \& Youth Care, Leiden University, Leiden, The Netherlands, ${ }^{7}$ Department of Psychology, Humboldt-Universität zu Berlin, Berlin, Germany, ${ }^{8}$ Institute 
of Neurosciences, Hospital Clinic, CIBERSAM, Barcelona, Spain, ${ }^{9}$ Department of Psychology, Education and Child Studies, Erasmus University Rotterdam, Rotterdam, The Netherlands, ${ }^{10}$ Department of Psychiatry, Leiden University Medical Center; Leiden Institute for Brain and Cognition, Leiden, The Netherlands, ${ }^{11}$ Department of Anatomy and Neurosciences, Amsterdam UMC, Location VUMC, Amsterdam, The Netherlands, ${ }^{12}$ Orygen \& Centre for Youth Mental Health, University of Melbourne, Melbourne, Australia, ${ }^{13}$ Imaging Genetics Center, Mark and Mary Stevens Neuroimaging and Informatics Institute, Keck School of Medicine, University of Southern California, Los Angeles, United States of America, ${ }^{14}$ South African Medical Research Council Unit on Risk \& Resilience in Mental Disorders, University of Cape Town, Cape Town, South Africa

Background: There is limited convergence in findings of brain magnetic resonance imaging (MRI) studies of anxiety disorders. Small sample sizes have limited the power of many prior investigations, and clinical and analytic heterogeneity have limited the replicability of findings. To address these concerns, the ENIGMA (Enhancing Neuro-Imaging Genetics through Meta-Analysis)-Anxiety Working Group initiated multiple large multi-cohort comparisons of brain structure between individuals with an anxiety disorder and healthy controls. Four subgroups were established, studying generalized anxiety disorder (GAD), social anxiety disorder (SAD), panic disorder (PD), and specific phobia (SP); as reviewed by BasHoogendam, Groenewold et al (Human Brain Mapping, 2020). At present, over 200 investigators from institutes worldwide are involved as members of ENIGMA-Anxiety.

Update: In the GAD-subgroup, subcortical and cortical brain structure were compared between 1,112 individuals with GAD and 3,282 healthy controls. A GAD-by-sex interaction for right ventral diencephalon volume was identified in a secondary analysis (Harrewijn et al., in revision). A greater ventral diencephalon volume was observed in male individuals with GAD $(n=359)$ compared to male controls $(n=1,477)$, whereas female individuals with GAD $(n=753)$ did not differ from female controls $(n=1,805)$.

In the SAD-subgroup, volumes of subcortical brain regions were compared between 1,120 individuals with SAD and 2,897 healthy controls. Here, a SAD-by-age interaction was found: adult individuals with SAD ( $n=668$, age range 22-69 years), but not adolescent individuals with $\operatorname{SAD}(n=437$, age range $8-21$ years), showed smaller putamen volumes compared to controls. Analyses on cortical thickness, cortical surface area and diffusion tensor imaging (DTI) metrics in SAD are ongoing.

In the PD-subgroup (900 individuals with PD) and SP-subgroup (1273 individuals with SP and 3415 controls), analyses of subcortical and cortical brain structure are in progress. Recently, two new subgroups were founded, focused on childhood inhibited temperament (as an innate risk factor for anxiety disorders) and on brain activation patterns underlying fear conditioning. Inhibited temperament data is presently being aggregated (anticipated sample size: 4000 subjects from 12 research groups), and 25 groups have expressed interest in contributing fear conditioning data. The ENIGMA-Anxiety Working Group is currently planning new projects, including transdiagnostic comparisons of resting-state functional connectivity and diagnostic classification based on machine learning algorithms in adults and youth with anxiety disorders.

Conclusion: The ENIGMA-Anxiety Working Group is a global enterprise at the intersection of mental health and neuroscience, aiming to advance understanding of the brain characteristics associated with anxiety disorders. This large-scale collaboration has identified subtle differences in brain structure in anxiety disorders and associated sources of heterogeneity through world-wide data sharing. ENIGMA-Anxiety provides an inclusive, multidisciplinary research environment that will continue to inspire new avenues of research.

\section{References}

Bas-Hoogendam, J.M., Groenewold N.A., et al. "ENIGMA-Anxiety working group: Rationale for and organization of large-scale neuroimaging studies of anxiety disorders." Human Brain Mapping (2020). Epub ahead of Print.

Harrewijn, A., et al. "Cortical and Subcortical Brain Structure in Generalized Anxiety Disorder - Findings from 28 research sites in the ENIGMA-Anxiety Working Group.” In revision.

\section{1 \\ Serotonin imbalance and disorders of emotional dysregulation}

\section{PD Dr. Angelika Schmitt-Böhrer}

\section{${ }^{1}$ University Clinic of Würzburg, Rimpar, Deutschland}

Serotonin (5-HT) shapes brain networks during development and modulates a wide spectrum of essential neuronal functions of the mature brain. Imbalances in 5-HT-moderated synaptic signaling is known to impact the pathophysiology, long term outcome, and the treatment response of psychiatric disorders with emotional dysregulation such as anxiety disorders and depression, but also ADHD.

Both, the 5-HT transporter (5-HTT) as well as the tryptophan hydroxylase-2 (TPH2), are key molecules of serotoninergic neurotransmission. The 5-HTT, responsible of the reuptake of synaptically released 5-HT into the presynaptic neuron, is the target of many anxiolytics and antidepressants. In humans, 5-HTT gene variants (e.g. the 5-HTTLPR) resulting in lower expression levels are associated with higher neuroticism scores and behavioral traits of anxiety. TPH2 catalyzes the first and rate-limiting step in the biosynthesis of neuronal 5-HT. Human variants of the TPH2 gene were shown to be associated with anxiety-, aggression- and depression-related personality traits and with the pathogenesis of disorders featuring deficits in cognitive control and emotion regulation.

In recent years it has become increasingly evident that neither genetic susceptibility nor environmental factors (for example stressful life events) alone provide a basis for the development of psychiatric disorders. Therefore, failure in cognitive control and emotional dysregulation, such as anxiety, depression, and aggression, are thought to be generated by a complex interaction of environmental factors with a number of gene products involving brain structures and circuits such as the 5-HT system.

Gender is a factor that is unfortunately often underestimated. It should also be considered when examining the genetic effects on psychological outcomes. For instance, males and females who carry the short 5-HTTLPR allele react to different environmental factors, and their response to environmental stress even seems to go in opposite directions. Moreover, a higher susceptibility to depressive reactions during tryptophan depletion was shown in women being homozygous with regard to the short 5-HTTLPR allele.

Investigations in animal models are thus needed to elucidate the underlying neural mechanisms as a basis for developing novel approaches for prevention and/or treatment of psychiatric disorders. In addition, animal models offer the possibility of taking a closer look at gene by environment $(\mathrm{GxE})$ interactions and gender differences in connection with a differently adjusted 5-HT system. For this mission 5-HTT knockout (KO) and TPH2 KO mice are perfect animal models as they show opposing neurochemical, neuroendocrine and behavioural phenotypes. 5-HTT deficient mice exhibit increased extracellular 5-HT concentrations, and TPH2 deficient mice lack 5-HT in the central nervous system. At the behavioral level 5-HTT KO mice exhibit, among other things, increased anxiety-like behavior and reduced aggressiveness, while TPH 2 KO mice are characterized 
by reduced anxiety-like behavior, increased impulsivity and increased aggressiveness. As shown in several studies, stress can aggravate or diminish these behavioral properties. Even if most animal GxE experiments were performed using male mice, some studies used both sexes. Behavioral results of these $\mathrm{GxE}$ studies point to an additional influence of gender as it has been suggested by human association studies mentioned above.

\section{2 \\ Longitudinal associations between sleep and stress in adolescence}

\author{
Dr Leila Tarokh \\ ${ }^{1}$ University Hospital of Child and Adolescent Psychiatry \\ and Psychotherapy, Bern, Switzerland, ${ }^{2}$ Translational Research \\ Center, University Hospital of Psychiatry and Psychotherapy, Bern, \\ Switzerland
}

An intuitive and intimate link exists between sleep and stress. Stressful life events can disrupt sleep, while disruptions in sleep can increase stress reactivity. While experimental studies, conducted primarily in animal models, have shown the impact of a stressor on sleep the longitudinal association between psychosocial stress and sleep is understudied. The current study takes a multimodal and longitudinal approach to examine the association between perceived stress and sleep in a sample of adolescents with and without depression. As part of this study, 18 adolescents with major depressive disorder (MDD) and 21 healthy controls (HC) between the ages of 14 and 17 years (mean $=15.15 ; \mathrm{SD}=1.1$ ) underwent one night of polysomnography (PSG) and reported on psychological stress using the perceived stress scale (PSS). A partial correlation controlling for age, sex and group (MDD vs HC) was used to correlate slow wave activity (SWA), a measure of sleep depth derived from the PSG and stress (total PSS score). Following the PSG night, participants filled out monthly online questionnaires including the PSS and the Pittsburgh Sleep Quality Index (PSQI), which used to measure sleep quality and quantity. On average 10.41 months of data were available across participants (range $=2-15$ months). Cross-lagged panel analyses were used to examine the association between sleep and stress over time. Examining the association between sleep EEG and stress, we find that lower levels of SWA were associated with more stress over temporal and parietal brain regions. With regards to the temporal association between stress and sleep, we find a bi-directional relationship wherein higher stress levels in the past month predicted shorter sleep duration in the following month $(\beta-0.04, \mathrm{p}<.001)$, and conversely, reduced subjective sleep duration was predictive of higher stress $(\beta=-0.06, p<.01)$. On the other hand, stress and subjective sleep quality were uni-directionally related with higher stress scores predicting future lower subjective sleep quality $(\beta=$ $0.03, p<.001)$ only. In a sample of adolescents with and without depression, we find cross-sectional and longitudinal associations between stress and sleep. Our findings are in line with previous results in animal models and humans showing diminished sleep depth associated with stress. In addition, our longitudinal study design allows for the elucidation of associations that persists over time. Considering these long-term associations as well as the essential role of sleep for development, our results underline the importance of both stress reducing and sleep improving interventions to support mental and physical health in youth.

\section{3}

Effects of the COVID-19 lockdown on media-related behavior and mental health in adolescents with preexistent internalizing disorder

Dr. med. Anna Maria Werling ${ }^{1}$, Prof. Dr. med. Dipl.-Psych. Susanne Walitza ${ }^{1,2,3}$, Prof. Dr. Edna Grünblatt ${ }^{1,2,3}$, Prof. Dr. phil. Renate Drechsler ${ }^{1}$

${ }^{1}$ Department of Child and Adolescent Psychiatry and Psychotherapy, Psychiatric University Hospital Zurich, University of Zurich, , Switzerland, ${ }^{2}$ Zurich Center for Integrative Human Physiology, University of Zurich, , Switzerland, ${ }^{3}$ Neuroscience Center Zurich, University of Zurich and ETH Zurich, , Switzerland

Objective: To investigate the effects of the spring 2020 lockdown on media behavior and mental well-being in adolescent patients with preexistent internalizing disorder (ID) compared to patients with other psychopathological conditions in a clinically referred sample.

Method: Adolescents who had been treated in the Department of Child and Adolescent Psychiatry and Psychotherapy, University of Zurich, and their parents were invited in June 2020 to participate in an anonymous online survey on the effects of the spring lockdown and its associated social restrictions. The surveys consisted of two parts: A screening questionnaire on media use related behaviors (PUI-SQ) and questions related to COVID-19 effects on mental health in children and adolescents (CRISIS). Media use related behaviors had to be rated three times: retrospectively regarding behaviors before and during the lockdown and regarding the last 2 weeks.

Results: $\mathrm{N}=210$ patients from the parent survey and $\mathrm{N}=178$ patients from the patient survey aged 12-18 years were included in this study. About half of the patients presented internalizing disorders (ID), the other half externalizing disorders or other psychopathologies (non-ID patients). All information obtained on diagnoses was self-assessed. ID patients indicated significantly more often a deterioration of symptoms under lockdown compared to non-ID patients. All parents and patients indicated an important increase of media use under lockdown and a decrease after the release of measures. Parents of patients with ID indicated higher social media use (mean time per day) during the lockdown and they were more concerned about the effects of media use on their child's mental health than parents of patients with other psychopathologies. According to self-ratings, elevated social media time was related to improvement rather than deterioration of symptoms in ID, but not in non-ID patients.

Conclusion: Adolescents with internalizing disorders were particularly affected by lockdown measures and media use had an important impact on their condition.

\section{4}

\section{"Hot" new data from the PGC Anxiety Disorders Working Group}

Prof. Dr. John (Jack) Hettema ${ }^{1}$, Dr. Manuel Mattheisen, Dr. Rosa Cheesman, Nora Strom, Dr. Kirstin Purves, Brad Verhulst, SilviuAlin Bacanu, Thalia Eley, Jürgen Deckert

${ }^{1}$ Texas A\&m University Health Science Center, College Station, United States

Background: Anxiety Disorders (ANX) are highly prevalent and moderately heritable mental disorders. They often begin in childhood and ultimately occur in around $25 \%$ of the population across the lifespan. They are a particularly complex and heterogeneous group with high comorbidity, making attempts to identify susceptibility loci 
a challenge. Several prior ANX GWAS conducted in moderately sized samples provided preliminary reports of genome-wide significant signals. Since its formation in 2017, PGC-ANX has formed collaborations with over 30 research groups from 10 countries to conduct well-powered ANX GWAS.

Methods: Our most recent data freeze includes over 100K ANX cases and $500 \mathrm{~K}$ unaffected controls from over 25 individual cohorts. We conducted genome-wide analyses within cohorts and then metaanalysed. We applied Genomic SEM to examine consistency of signals across individual cohorts. We estimated SNP-based heritability within ANX cohorts and co-heritability between ANX and closely related phenotypes from other large studies. We performed additional analyses to examine gene-based associations and functional annotation of associated signals.

Results: Multiple novel genome-wide significant loci are identified using the meta-analysed samples. Exploratory factor analyses implemented in Genomic SEM support the existence of a common latent factor, indicating satisfactory consistency of signal across individual cohorts. We observed strong genetic correlations between ANX and neuroticism, major depression, and other ANX-related disorders. Gene-based and functional analyses complement and provide insight into SNP-based findings.

Conclusions: This largest GWAS for ANX was able to detect multiple genome-wide significant risk variants. As predicted from twin studies, ANX share some but not all of their genetic risk with depression and neuroticism.

\section{5}

\section{Genome-wide association studies by the psychiatric genomics Consortium for PTSD identify novel loci and consistent genetic signals across populations}

\author{
Dr. Caroline Nievergelt ${ }^{1,2}$, Adam Maihofer ${ }^{1,2}$ \\ ${ }^{1}$ University Of California San Diego, La Jolla, United States \\ of America, ${ }^{2}$ Center of Excellence for Stress and Mental Health, \\ Veterans Administration, San Diego, United States of America
}

Background: First replicable PTSD risk loci have been identified by large-scale meta-analyses of genome-wide association studies (GWAS) conducted by the Psychiatric Genomics Consortium PTSD workgroup (PGC-PTSD). While case-control studies are the standard study design for PGC analyses, we found that incorporation of PTSD symptom severity and measures on trauma exposure increased power to identify PTSD risk loci. However, risk variants identified by the largest PTSD GWAS from PGC and by the Million Veteran Program (MVP) published to date represent only a fraction of the overall genetic liability, and the vast majority of risk loci has yet to be identified. Furthermore, the degree to which genetic risk is shared across diverse populations at risk for developing PTSD remains to be investigated.

PGC-PTSD has expanded data collection and currently includes over 1.25 million subjects (Freeze 3), with 80,000 participants of diverse non-European ancestry. Data sources include $>90$ studies with heterogeneous PTSD assessments based on clinical diagnoses, selfreported questionnaire data, and PTSD diagnostic status from electronic health records in biobanks (EHR). We present a first look at these data and present GWAS and post-GWAS analyses that form the most comprehensive picture of PTSD genetics to date.

Methods: GWAS on PTSD diagnosis or symptom scores were performed stratified by cohort and ancestry group and sample-size weighted fixed effects meta-analysis was performed using METAL. Heritability and genetic correlation were estimated using cov-LD score regression. Functional annotation of findings was performed using FUMA. Univariate and bivariate mixer model (MiXeR) was used to estimate polygenicity and discoverability in the three main PTSD data sources PGC-PTSD cohorts, N $\sim 220 \mathrm{~K}$ subjects; MVP, N $\sim 215 \mathrm{~K}$; electronic health records (EHR); N $820 \mathrm{~K}$ ), and to quantify polygenic overlap between PTSD and other psychiatric disorders.

Results: Meta-analysis in European ancestry identified over 50 genome-wide significant loci $(\mathrm{p}<5 \mathrm{e}-8)$, with some evidence for replication in non-European ancestries. SNP based heritability was approximately $6-8 \%(\mathrm{p}<1 \mathrm{e}-50)$ and comparable between the three main PTSD data sources. An estimated 7671 (in MVP), 10,340 (in PGC-PTSD cohorts), and 10,418 (in EHR) causal loci ( $<<1 \mathrm{e}-20$ ) explained most $(90 \%)$ of this variation. Polygenicity and discoverability of PTSD was comparable to other psychiatric disorders, most closely to MDD. Correspondingly, bivariate analyses showed high genetic correlations and broad polygenic overlap between PTSD and other psychiatric disorders, in particular MDD ( $\mathrm{rg}=0.85$ ). Further analysis of the shared variants between PTSD and MDD show considerable differences in effects (mean heterogeneity statistic chi $2=$ 1.12).

Conclusions: The overall genetic signal for PTSD across diverse data sources and populations is fairly consistent, despite heterogeneity in assessment, ascertainment, and differences in trauma exposure. We identified novel PTSD risk loci, with some providing evidence of trans-ethnic replication. Gene-based analyses have started to enhance our understanding of PTSD biology. However, substantially more loci have yet to be identified to inform our understanding of PTSD biology and next steps will focus on analyses delineating PTSD from highly correlated psychiatric disorders.

\section{6}

Stress and anxiety among children/adolescents, their parents and young adults during the first COVID-19 lockdown in Switzerland

\section{Prof. Dr. Meichun Mohler-Kuo ${ }^{1}$}

${ }^{1}$ University Hospital Of Psychiatry Zurich Department Of Child And Adolescent Psychiatry And Psychotherapy, Zurich, Switzerland, ${ }^{2}$ La Source, School of Nursing Sciences, HES-SO University of Applied Sciences and Arts of Western Switzerland, Lausanne, Switzerland

\section{Abstract}

Introduction: The outbreak of emerging infectious diseases like COVID-19 often have a psychological impact on the well-being of the general population due to the fear of disease itself and social isolation due to containment measures. The present study aims to assess various stressful situations and the psychological impact of the first Covid-19 pandemic lockdown among youths in Switzerland. Methods: We included samples of 1627 young adults from the Swiss Youth Epidemiological Study on Mental Health (S-YESMH) and 1146 children and adolescents ages 12-17 years and their parents. We assessed various symptoms of mental disorders (depression, anxiety and ADHD), problem internet use (PUI), perceived stress and coping strategies during the first Covid-19 lockdown. In addition, we examined the direct and indirect associations among these variables using Structural equation modeling (SEM).

Results: During the first lockdown in Switzerland, the most common sources of perceived stress were disruption of social life and important activities, uncertainty about how long the state of affairs would last, and the pandemic itself. In addition, around one fifth of the young adults met the criteria of one of the symptoms of mental disorders (depression, anxiety or ADHD), while one third of children/ adolescents screened positive for one of the mental health problems 
(depression, anxiety, ADHD or ODD). Moreover, $30.1 \%$ of children and $21.3 \%$ of young adults met the criteria of PUI. We found high correlations between stresses, ADHD symptoms, PUI and anxiety among children and adolescents. The path analyses using SEM showed that ADHD symptoms have a significant direct effect on PUI and anxiety respectively. In addition, ADHD symptoms have an indirect effect on anxiety mediated through problem internet use. After including Covid-19-related stressful situations in the model, we found that parents' and children' stress was positively and highly correlated. Parents' stress indirectly affected children's anxiety through children's stress. In addition, children's stress during the Covid-19 lockdown had a direct effect on children's ADHD symptom, PUI and anxiety. Children's Covid-19-related stress also had a significant indirect effect on children's anxiety through ADHD and problem internet use.

Conclusion: The study showed considerable stresses perceived by young adults and symptoms of mental disorders, especially among females during the first Covid-19 lockdown in Switzerland.Stressful situations were positively associated with ADHD, PUI and anxiety among children and adolescents. The implication for parents and clinicians will be discussed.

\section{7}

\section{Traditional Japanese medicine in anxiety and depression}

\section{Professor Makoto Naoi ${ }^{1}$ \\ ${ }^{1}$ Department of Health and Nutrition, Faculty of Psychological and Physical Science, Aichi Gakuin University, Aichi, Japan}

Anxiety, mood, sleep disorders and chronic fatigue are prevalent morbid psychiatric conditions. Among the patients treated with conventional anxiolytics and antidepressants, treatment with herbal medicine and Complementary and Alternative Medicine (CAM)is widespread. In China, Japan, Korea, Malaysia and Vietnam, traditional Chinese medicine (TCM), especially traditional herbal medicine, had been major theoretical and practical basis of medicine. Kampo 漢方 (traditional Japanese medicine) means methods or formulae originated at the period of the Han (漢) dynasty (206 BC to 220 AD) of early China and transported in Japan around the 5th century. Kampo has been developed as Japan-specific disease concepts and medicine during the Edo period (17th to 18th century).

Based on the philosophical principle of TCM of "Yin-Yang" balance and three substances, Qi 気 (vital energy), Blood and Water (quite different from common usage), external and internal stressful insults disturb circulation of Qi, cause Qi excess in liver, called "Liver (not the anatomical concept) stagnation", and undesirable state of "Qi" within the body. The unbalance in harmony of mind and body induces "Constraint" 㯒, which has been used to express the emotional, psychological and physical sufferings, and may correspond to stress-induced emotional disorders in Western medicine. Kampo formulae are prepared from mixed species of herbs, and now used commonly as lyophilized powder of water extract of herbs. To exert anxiolytic effects, Kampo formulae normalize deregulation of the hypothalamic-pituitary- adrenal axis (HPA) and normalize disturbed function of monoamines and GABA. Effective ingredients of Kampo herbs include alkaloids, terpenes, flavonoids, phenolic acids, saponins and others.

Herbal medicine has been traditionally used for anxiety and related disorders in the world as CAM. Mechanisms behind anxiolytic and antidepressant functions of herb ingredients have been investigated in animal and cellular models. Anxiolytic functions of phytochemicals, especially flavonoids and phenolic acids, have been clarified. Flavones, ferulic acid, hyperforin and others inhibit type A monoamine oxidase (MAO-A) and increase serotonin, noradrenaline and dopamine levels. Ginsenoside, curcumin and genistein increase expression of serotonergic receptors (5-HT1A) in the hippocampus. 6-Hydroxyflavone and related derivatives, EGCG, baicalin, kavalactone are agonists of GABAA receptors. 7,8-Dihydroxyflavone binds to TrkB receptor specific brain-derived neurotrophic factor (BDNF) and activates intracellular signaling pathways to induce BDNF, promotes neurogenesis and alleviate neurodegeneration and maintain synaptic plasticity in the hippocampus and locus coeruleus.

In Japan, Kampo medicine has been included into the National Health Insurance System. Now about $83 \%$ physicians prescribe Kampo medicines as the adjuncts of convenient anxiolytics and antidepressants, corresponding to subjective symptoms of patients. Kampo medicine is required for more scientific evidence-based basic and clinical studied in order to understand the molecular mechanisms from the etiological, pathological and pharmacokinetic aspects.

108

Stress and myelin plasticity: animal model data on anxiety-like behavior

\section{Prof. Dr. Iiris Hovatta ${ }^{1}$ \\ ${ }^{1}$ University Of Helsinki, Helsinki, Finland}

Chronic psychosocial stress is a well-established risk factor for anxiety disorders. Mechanisms by which chronic stress impacts susceptibility and resilience to psychiatric disorders are largely unknown. The chronic social defeat stress (CSDS) mouse model allows identification of factors underlying resilience and susceptibility to chronic psychosocial stress, in a controlled manner not feasible in human settings. We first investigated the effect of genetic background on behavior and brain gene expression patterns after CSDS. We identified a strong genetic background effect in CSDS-induced social avoidance using four inbred mouse strains: $69 \%$ of $\mathrm{C} 57 \mathrm{BL} / 6 \mathrm{NCrl}$ (B6), $23 \%$ of BALB/cAnNCrl, $19 \%$ of $129 \mathrm{~S} 2 / \mathrm{SvPasCrl}$, and $5 \%$ of DBA/2NCrl (D2) mice were stress-resilient. To identify biological pathways affected by CSDS, we used RNA-sequencing of three brain regions of two strains, B6 and D2: medial prefrontal cortex (mPFC), ventral hippocampus (vHPC), and bed nucleus of the stria terminalis. We surprisingly discovered an over-representation of oligodendrocyte-related genes in the differentially expressed genes. Because oligodendrocytes myelinate axons, we used electron microscopy to measure myelin thickness and found significant brain region and strain-specific differences. For example, in resilient D2 mice mPFC axons had thinner myelin than controls, whereas susceptible B6 mice had thinner myelin than controls in the vHPC. Neither myelin-related gene expression in several other regions, nor corpus callosum thickness, differed between stressed and control animals, suggesting that myelination changes are specific to stress-related brain regions. Myelin plasticity, or adaptive myelination, encompasses various alterations in myelin structure, density, and function in response to specific experiences and consequent changes in neuronal activity. Using diffusion tensor imaging to investigate myelination in humans, we documented larger mean diffusivity in the forceps minor in young adults with anxiety symptoms compared to those without. Our unbiased genetic screen suggests that myelin plasticity is one of the major responses to chronic psychosocial stress in mammals, varies across 
brain regions, and is genetically controlled. Identification of the mechanisms underlying the myelin response will provide mechanistic insight into the molecular basis of anxiety, a critical step in developing targeted therapy.

\section{9}

\section{Positive self-instruction increases self-esteem and well- being following negative social evaluation}

\section{Dr. Lydia Kogler ${ }^{1}$ \\ ${ }^{1}$ General Psychiatry and Psychotherapy University Hospital Tübingen, Tübingen, Germany}

In our daily lives we are permanently confronted with social evaluation. Negative social evaluation can decrease self-esteem, which is a transdiagnostic symptom amongst mental disorders. Inner self-speech has a self-regulatory function, such as controlling affective reactions. However, this inner self-speech is often negative and evaluative, thereby inducing low self-esteem, fostering anxiety and depression. With the current study we modified inner self-speech via positive selfinstruction to modulate subjective and neural reactions to negative social feedback in 63 healthy women. Results show a general increase in self-esteem in the intervention-group $(n=33)$ compared to the control-group $(\mathrm{n}=30)$ and less subjective arousal during the negative social feedback paradigm. This was accompanied by decreased activation in the anterior cingulate cortex (ACC) after the training for positive self-instruction. Thus, our results suggest that positive selfinstruction significantly modifies self-esteem and subjective negative reactions to negative social evaluation. Decreased neural activation in the ACC potentially reflects self-regulatory processes. Fear of negative evaluation and inadequate dealing with situations of social evaluative is prevalent in various mental disorders. Our intervention is a promising tool to increase self-esteem and to reduce subjective (maladaptive) reactions to stressful situations such as negative social evaluation.

\section{0}

Cognitive test performance in relation to omega-3 fatty acids in pediatric Major Depression

\author{
Dr. Sophie Emery ${ }^{1}$, Isabelle Häberling ${ }^{1}$, Gregor Berger ${ }^{1}$, Mona \\ Albermann $^{1}$, Susanne Walitza ${ }^{1,2,3}$, Renate Drechsler ${ }^{1}$ \\ ${ }^{1}$ Department of Child and Adolescent Psychiatry and Psychotherapy, \\ Psychiatric Hospital, University of Zurich, Zurich, Switzerland, \\ ${ }^{2}$ Neuroscience Center Zurich, University of Zurich and ETH Zurich, \\ Zurich, Switzerland, ${ }^{3}$ Center for Integrative Human Physiology \\ Zurich, University of Zurich, Zurich, Switzerland
}

Animal studies have demonstrated the involvement of polyunsaturated omega-3 fatty acids (n-3 PUFAs) in several neuronal processes like neurotransmission, myelination or neuroinflammation. Research in humans has also confirmed their vital role in human neurodevelopment. These findings together have generated considerable interest in investigating potential cognitive effects of n-3 PUFAs and some studies have indeed described n-3 PUFAs to be positively associated with cognitive functioning. Meta-analytic findings to date are heterogeneous but have identified eicosapentaenoic acid (EPA) as potentially more effective than docosahexaenoic acid (DHA). Children and adolescents with major depression might possibly benefit from this effect, as cognitive impairments have been described in this population. In our study, patients with moderate to severe pediatric Major Depression, mean age 15.5 years, performed a list learning and a working memory task. Test results were analysed with regard to red blood cell (RBC) DHA and EPA levels and depression severity. While patients with higher EPA level performed better on the list learning task, the DHA level had no effect on test performance. Results were not affected by depression severity. In consequence, EPA but not DHA levels seem to have an impact on cognitive functioning in depression. The differential effects of EPA compared to DHA on cognition should be investigated in further studies.

Publisher's Note Springer Nature remains neutral with regard to jurisdictional claims in published maps and institutional affiliations. 